岂

Interaction Between Beam Control and RF Feedback Loops for High $Q$ Cavities and Heavy Beam Loading

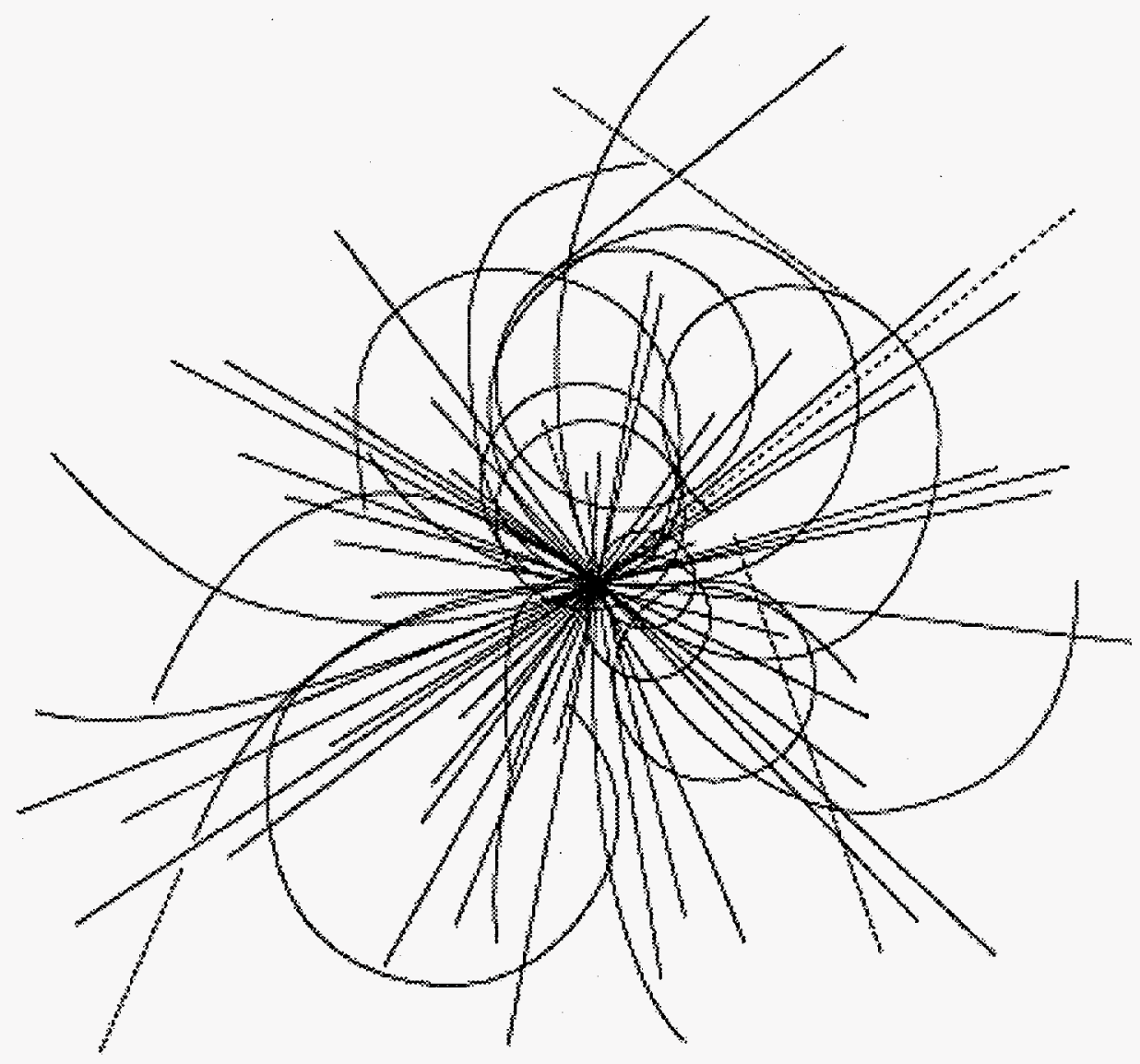
L.K. Mestha
C.M. Kwan
K.S. Yeung
L.K. Mestha
C.M. Kwan
K.S. Yeung
L.K. Mesth
C.M. Kwan
K.S. Yeung

SSCL-Preprint-195 Rev. A April 1994

Distribution Category: 414 


\section{Disclaimer Notice}

This report was prepared as an account of work sponsored by an agency of the United States Government. Neither the United States Government or any agency therecf, nor any of their employees, makes any warranty, express or implied, or assumes any legal liability or responsibility for the accuracy, completeness, or usefuiness of any information, apparatus, product, or process disclosed, or represents that its use would not infringe privately owned rights. Reterence herein to any specific commercial product, process, or service by trade name, trademark, manufacturer, or otherwise, does not necessarily constitute or imply its endorsement, recommendation, or favoring by the United States Government or any agency thereot. The views and opinions of authors expressed herein do not necessarily state or reflect those of the United States Government or any agency thereot.

Superconducting Super Collider Laboratory is an equal opportunity employer. 


\section{DISCLAIMER}

Portions of this document may be illegible in electronic image products. Images are produced from the best available original document. 
SSCL-Preprint-195 Rev. A

\title{
Interaction Between Beam Control and RF Feedback Loops for High Q Cavities and Heavy Beam Loading*
}

\author{
L.K. Mestha and C.M. Kwan \\ Superconducting Super Collider Laboratory ${ }^{\dagger}$ \\ 2550 Beckleymeade Ave. \\ Dallas, TX 75237
}

\section{K.S. Yeung}

University of Texas

Arlington, TX 76019

April 1994

*To be submitted to the "Particle Accelerators Journal."

†Operated by the Universities Research Association, Inc., for the U.S. Department of Energy under Contract No. DE-AC35-89ER40486. 


\title{
INTERACTION BETWEEN BEAM CONTROL AND RF FEEDBACK LOOPS FOR HIGH Q CAVITIES AND HEAVY BEAM LOADING
}

\author{
L. K. MESTHA AND C.M. KWAN \\ Superconducting Super Collider Laboratory, * Dallas, Texas 75237 \\ K.S. YEUNG \\ University of Texas, Arlington, Texas 76019
}

An open-loop state space model of all the major low-level rf feedback control loops is derived. The model has control and state variables for fast-cycling machines to apply modern multivariable feedback techniques. A condition is derived to know when exactly we can cross the boundaries between time-varying and time-invariant approaches for a fast-cycling machine like the Low Energy Booster (LEB). The conditions are dependent on the $\mathrm{Q}$ of the cavity and the rate at which the frequency changes with time. Apart from capturing the time-variant characteristics, the errors in the magnetic field are accounted in the model to study the effects on synchronization with the Medium Energy Booster (MEB). The control model is useful to study the effects on beam control due to heavy beam loading at high intensities, voltage transients just after injection especially due to time-varying voltages, instability thresholds created by the cavity tuning feedback system, cross coupling between feedback loops with and without direct rf feedback etc. As a special case we have shown that the model agrees with the well known Pedersen model derived for the CERN PS booster. As an application of the model we undertook a detailed study of the cross coupling between the loops by considering all of them at once for varying time, $Q$ and beam intensities. A discussion of the method to identify the coupling is shown. At the end a summary of the identified loop interactions is presented.

* Operated by Universities Research Association, Inc., for the U.S. Department of Energy under Contract No. DE-AC35-89ER40486. 
There are several feedback loops associated with the complete low-level rf system. There are high-bandwidth cavity amplitude and phase loops and a low-bandwidth tuning loop all of them local to the cavity. The radial loop, beam phase loop and synchronization loop are global to the ring accelerating system. For high-Q rf cavities the high-bandwidth local phase loop and amplitude loops may interact with the beam phase loop. It is a general practice in the accelerator community to design these loops as though there is only one loop acting on the machine in isolation and then collectively put them together to operate the machine with beam. It may so happen that the control signal from the local cavity phase loop has a significant effect on the beam phase which in effect may act against the function of the beam phase loop. Similarly, the control from the cavity amplitude loop may effect the beam phase loop at some frequencies below or above synchrotron frequencies. These effects are hard to see when all the loops are combined. Also, the loop interactions may be dominant when a high-Q cavity system is used to operate the machine at high intensities. Our aim in this paper is to show a method to identify the loop interactions on a highly-coupled system like the Low Energy Booster RF system and then, later in the paper, discuss the effects due to variable $Q$ (by including direct rf feedback) and variable beam intensities. Also, we discuss several methods to decouple the loops so that eventually each loop can be designed as single loop.

To study a coupled system, a good mathematical model of the beam feedback system with cavity dynamics is extremely useful. Otherwise, we have to rely on the measurement data on an operating machine which becomes virtually difficult to acquire on a time-varying machine. The complete analytical system model gives information such as the open-loop bandwidth, gain and phase values at different frequencies for each loop. Especially, a state-space model is better since the additional disturbance terms which are the functions of the cavity and machine parameters can be observed and compensated in the loops. In the literature we see work related to the development of a transfer function model by Pedersen ${ }^{1}$ in the 1970 s and subsequently by other authors. ${ }^{2,3}$ We see that a control model for fast-cycling machines was not obtained to integrate all the loops including the local phase loop planned for the Low Energy 
Booster (LEB). In the initial stages of this paper an open-loop state-space model of the control loops is derived. Our model has all control and state variables for fast-cycling machines to apply modern multivariable feedback techniques. As a special case the model is compared with Pedersen's model for a time-invariant machine. After deriving the model we show the normalization techniques and then the coupled transfer characteristics with respect to frequency at a given time during acceleration for conditions with direct rf feedback and then for varying beam currents. The information will be useful to design a complete decoupled low-level rf system.

\section{DESCRIPTION OF THE LEB RF BEAM CONTROL LOOPS}

Several feedback loops are associated with the complete low-level rf system. The goal of the system is to be able to bunch the beam, accelerate and extract to the higher-energy machine. To be able to do this a precise control of the frequency, phase, and amplitude of the gap voltage is required. In Figure 1 we show the complete feedback loops associated with the LEB with two cavities to represent the signal distribution. Loops can be divided based on their functions. Loops local to each of the rf stations are called local cavity loops in this paper. Also, those loops controlling the frequency of the drive rf signal by sensing the beam information are called global loops.

\subsection{Feedback Loops Local to Each Cavity}

There are four feedback loops shown local to each cavity: a direct rf feedback loop, a cavity tuning loop, a local phase loop, and a local amplitude loop. The direct rf feedback loop consists of summing the processed gap voltage signal with the signal before the power amplifier as

shown in Figure 1. This loop has the effect to reduce the effective $Q$ of the cavity. ${ }^{4,5,6,7}$ The local phase loop in essence consists of a phase detector (PD), a feedback controller, and a phase shifter $\delta \phi^{c}$. The phase detector measures the phase difference between the rf signal supplied from the global frequency control unit and the measured gap voltage. The phase loop operates by changing the phase of the generator current at the rf signal frequency driving the power amplifier. It is included to maintain the cavity gap voltage at the phase set by the rf drive signal 
from the global frequency control unit. The local amplitude loop changes the amplitude of the generator current to maintain the error between the envelope of the gap voltage signal and the reference voltage signal to within specification. Thus, the local amplitude loop maintains the required amplitude on the gap voltage signal. Both amplitude and phase loops are particularly more effective at high beam intensities. The open-loop bandwidths of these loops are important and depend on the cavity and machine parameters. The cavity tuning loop shown in Figure 1 consists of a phase detector to measure the phase difference due to tuning error. This signal is fed to the Tuning Bias Regulator (TBR) through a controller driving the ferrite tuner to correct for the tuning error. The set point is required to add a detuning profile after the beam is injected into the machine. It also helps for arranging additional feedforward correction needed for the tuning loop. The tuning loop is generally slow due to the cost involved in building fast tuners.

\subsection{Feedback Loops Global to the Cavity}

The global feedback loops have the special task of controlling the beam to capture and accelerate while correcting for radial excursions due to field errors. The synchronization of the beam bunches from the LEB to the MEB is another requirement. The global loops are: radial loop, beam phase loop, and synchronization loop. Interconnection of these loops is shown in Figure 1. A stable frequency source such as the Direct Digital Synthesizer (DDS) is used for producing a sinusoidal if signal by way of reading frequency ramp values from a table. The frequency ramp table is inexorable and needs corrections to minimize errors in beam orbit or coherent beam oscillations around the synchronous phase. In the radial loop, the radial position of the beam is compared to a preset steering reference signal to generate the radial error signal. The radial error signal is processed and then the resulting signal adds or subtracts the frequency ramp values to maintain the radial error to within the specifications. In the beam phase loop, the beam position signal is compared directly from a wall-current monitor in the beam phase detector. It is then processed to convert to the appropriate frequency shift through a feedback controller. The frequency shift is added to the frequency ramp values. The synchronization loop is configured depending on the approach needed. In the trip-plan scheme, ${ }^{8}$ the time interval between the LEB and the target MEB markers is measured preferably using a 
Time-to-Digital Converter (TDC) at the time the target MEB marker appears. The data is then compared with a table and processed to generate the frequency shift to the ramp values. In this way locking can be achieved to predetermined values so that the synchronism is guaranteed. In addition to the loops we have described, there could be loops to damp the quadrupole oscillations. Also, one-turn delay loops to correct the effects on cavities due to gaps in the bunch train are commonly used in many accelerators. ${ }^{9}$ The global paraphase hardware, as shown in Figure 1, is also used to maintain low effective gap voltage at the time of injection.

All the active loops are important for the operation of the machine. Their design is highly complex and it becomes even more complicated when some of the loops are coupled. To ensure global stability of the loops and also meet the specifications on gap voltage phase and amplitude, it will be useful to know the coupling between them. The 'coupling' means: say for example, the control of a local phase loop (phase shift of the rf drive signal in the local rf station) affecting the beam phase error or vice versa. Such things are very hard to see unless a complete system model is derived. Especially, a state-space model is better since the additional disturbance terms which are the functions of the cavity and machine parameters can be observed and compensated in the loops. We have, therefore, actively pursued a rigorous mathematical approach in deriving the control model which is shown below.

\section{GLOBAL AND LOCAL CONTROL MODEL}

At first, a general control model to include all the feedback loops is derived by using an equivalent circuit model for the cavity. Later, we show a simplified reduced order model in analytical form. Comparisons are made with a special particle tracking code developed at the Superconducting Super Collider Laboratory (SSCL) to test the validity of the model.

\subsection{Cavity Control Model}

Consider a parallel equivalent RLC circuit for the cavity (Figure 2). Then the following differential equation can be written for the total gap voltage, $v$, with $\omega_{R}$ as the resonant frequency. 


$$
\frac{d^{2} v}{d t^{2}}+2 \sigma \frac{d v}{d t}+\omega_{R}^{2} v-\left[\frac{\omega_{R}^{3} Q}{R} \frac{d}{d t}\left(\frac{R}{\omega_{R} Q}\right) \int v d t+\frac{\omega_{R} v}{R Q} \frac{d R}{d t}\right]=2 \sigma R \frac{d i_{t}}{d t}
$$

where

$$
\begin{aligned}
& \sigma=\frac{\omega_{R}}{2 Q}=\text { Damping ratio } \\
& i_{t}=i_{g}+i_{b} .
\end{aligned}
$$

The terms within the square bracket have resulted due to time-varying frequency during simplification of the RLC equivalent circuit model. For the parameters of the LEB, this term can be ignored. Hence we use the following simplified model

$$
\ddot{v}+2 \sigma \dot{v}+\omega_{R}^{2} v=2 \sigma R \dot{i}_{t}
$$

The gap voltage, $v$, generator current $i_{g}$ and the beam current $i_{b}$ can be represented in terms of their fundamental components as below

$$
\begin{aligned}
v(t) & =V_{1} e^{j \int \omega d t} \\
i_{g}(t) & =I_{g 1} e^{j \int \omega d t} \\
i_{b}(t) & =I_{b 1} e^{j \int \omega d t} .
\end{aligned}
$$

The amplitudes $V_{1}, I_{g 1}$ and $I_{b 1}$ respectively for the gap voltage, generator current and beam current will be regarded as phasors in the analysis to follow. The term, $e^{j \int \omega d t}$, represents the phase due to frequency of the driving rf power which is time varying in fast-cycling machines. Generally, for studies related to beam loading instabilities, the exponent term, $e^{j \omega t}$, was used in the literature. ${ }^{2}$ This representation is valid, when the frequency of the rf signal is not varying with time to see significant differences in the stability of the feedback loops. However, for studies related to global stability of the loops from injection, throughout acceleration and until extraction we need to use a more accurate representation of the model since the frequency begins to ramp at a considerably faster rate for the LEB. In Appendix A, we derive three simple 
inequalities to show when actually the phase is represented with the exponent having the integral term. One of the inequalities, $\frac{\sigma}{\omega} \gg \frac{\dot{\omega} t}{\omega}$, is not satisfied for the LEB. Hence the representation shown in Eq. (3) is more accurate for the LEB.

\subsubsection{Cavity Voltage and Phase Model. The formulation of the voltage and current phasors} shown in Eq. (3) leads to an accurate control model which is different from that shown in the literature ${ }^{2}$ due to the time-varying nature of the frequency. To determine the representation of the amplitudes $V_{1}, I_{g 1}$ and $I_{b 1}$ in Eq. (3) we need to identify the measurable signals and control signals used for all the feedback loops around the cavity. Figure 3 shows the line diagram of the loop interface points of Figure 1. The "controller" of Figure 3 can be modeled with a simple gain or modeled with additional linear/nonlinear dynamics. The measured quantities (without any superscript) are shown symbolically pointing to the controller of each loop and the control parameters are shown with a superscript ' $c$ '. Also the direct $\mathrm{rf}$ feedback is represented with a feedback gain of $K_{d}$. By assuming the power amplifier as a high-bandwidth system with a pure gain of $K_{g}$, we can rewrite the equation for the generator current, $I_{\mathrm{g}}{ }^{\prime}$, as follows

$$
i_{g}^{\prime}=\left(i_{g}-K_{d} v\right) K_{g}
$$

where $i_{g}$ is the rf signal derived after applying appropriate frequency, amplitude, and phase correction from the local and global feedback loops. Substituting Eq. (4) into (1) we obtain

$$
\ddot{v}+2 \sigma H \dot{v}+\omega_{R}^{2} v=2 \sigma R K_{g} \dot{i}_{t}
$$

where

$$
\begin{gathered}
H=1+R K_{d} K_{g}, \\
i_{t}=i_{g}+\left(i_{b} / K_{g}\right)=i_{g}+\tilde{i}_{b} .
\end{gathered}
$$

Whereas with direct-rf feedback the generator current becomes, $i_{g}=\left(H / K_{g}\right) i_{g}$ and the steady-state generator current $I_{g}=H\left(V / R K_{g}\right)$ for the case without beam loading compensation. 
$R$ is the equivalent shunt resistance of one cavity and $K_{g}$ is the equivalent gain of the power amplifier driving one accelerating cavity. Clearly, the increase in the loop gain in the direct rf feedback loop and the power amplifier gain have a direct effect in reducing the effective $Q$ of the cavity. The generator current, $i_{g}$ can be regarded as the rf signal which will be combined with the direct rf feedback loop to generate the new rf signal driving the power amplifier. It must be noted at this stage that with the presence of direct rf feedback the generator current, $i_{g}$, at the summing point is increased by the factor $H$.

In Figure 4, a phasor diagram is shown to represent the relations among the currents and voltages at a given time for the machine operating at below transition. Under steady-state conditions the phase between the cavity gap voltage and the generator current is equal to $\phi_{L}$-the loading phase angle ${ }^{1}$ which is equal to the cavity impedance phase angle for no beam. $\phi_{z}$ is the phase angle of the total current (generator current, $i_{g}+$ fundamental beam current, $\tilde{i}_{b}$ ) with respect to gap voltage. The phase $\delta \phi_{\nu}$ is the transient perturbation in the cavity gap voltage acting as phase modulation over the steady-state voltage $V_{\text {ideal }}$. A local phase loop will maintain $\delta \phi_{v}$ to zero during normal operation. The phase modulation $\delta \phi^{c}$ is applied to the steady-state generator current in the local phase loop to maintain $\delta \phi_{\nu}$ to zero. Since when the beam comes on, the amplitude of the cavity voltage changes by $\delta V, V=V_{\text {ideal }}+\delta V$, by modulating the amplitude of $i_{g}, \delta V$ is suppressed in the amplitude loop.

From Figure 3 it is clear that the generator current rf signal, $i_{g}$, is phase and frequency modulated by various feedback loops. Therefore, the dynamical representation of the generator current, $i_{g}$, is shown below in terms of the control parameters

$$
i_{g}=\left(I_{g}+\delta I^{c}\right) e^{j\left(\int\left(\omega+\delta \omega^{c}\right) d t+\phi_{L}+\delta \phi^{c}\right)} .
$$

Similarly, the fundamental of the beam current and the cavity gap voltage is represented as follows 


$$
\begin{aligned}
& \tilde{i}_{b}=\tilde{I}_{b} e^{j\left(\int\left(\omega+\delta \omega^{c}\right) d t-\left(\frac{\pi}{2}+\phi^{s}+\delta \phi^{s}\right)\right)} \\
& v=(V+\delta V) e^{j\left(\int\left(\omega+\delta \omega^{c}\right) d t+\delta \phi_{\nu}\right)} .
\end{aligned}
$$

Where $\delta \omega^{c}$ is the sum of all the frequency shifts in radians per second applied from the global control loops as shown in Figure 3. Parameters of the tuning loop model will be included later. The currents $i_{g}$ and $\tilde{i}_{b}$ are differentiated once with respect to time and the gap voltage is differentiated twice with respect to time. The resulting derivatives of the current and voltage phasors are substituted in the modified equivalent circuit Eq. (5) above. After some simplification, real and imaginary parts are equated with those on the right-hand side. As a result, the following two nonlinear equations are obtained:

$$
\begin{gathered}
\ddot{\tilde{V}}-\tilde{V}\left(\delta \dot{\phi}_{v}\right)^{2}+2 \sigma H \dot{V}-2\left(\omega+\delta \omega^{c}\right) \tilde{V} \delta \dot{\phi}_{v}+\left[\left(\omega_{R}+\delta \omega_{R}\right)^{2}-\left(\omega+\delta \omega^{c}\right)^{2}\right] \tilde{V}=f_{1}, \\
\tilde{V} \ddot{\phi}_{v}+2(\dot{\tilde{V}}+\sigma H \tilde{V}) \delta \dot{\phi}_{v}+2\left(\omega+\delta \omega^{c}\right) \dot{\vec{V}}+\left[\dot{\omega}+\delta \dot{\omega}^{c}+2 \sigma H\left(\omega+\delta \omega^{c}\right)\right] \tilde{V}=f_{2},
\end{gathered}
$$

where

$$
\begin{aligned}
& f_{1}=2 \sigma R K_{g}\left\{\left(\dot{I}_{g}+\delta \dot{I}^{c}\right) \cos \left(\phi_{L}+\delta \phi^{c}-\delta \phi_{v}\right)-\right. \\
& \left(I_{g}+\delta I^{c}\right)\left(\omega+\delta \omega^{c}+\dot{\phi}_{L}+\delta \dot{\phi}^{c}\right) \sin \left(\phi_{L}+\delta \phi^{c}-\delta \phi_{v}\right) \\
& \left.\dot{\tilde{I}}_{b} \sin \left(\delta \phi_{v}+\phi^{s}+\delta \phi^{s}\right)+\tilde{I}_{b}\left(\omega+\delta \omega^{c}-\dot{\phi}^{s}-\delta \dot{\phi}^{s}\right) \cos \left(\delta \phi_{v}+\phi^{s}+\delta \phi^{s}\right)\right\}, \\
& f_{2}=2 \sigma R K_{g}\left\{\left(\dot{I}_{g}+\delta \dot{I}^{c}\right) \sin \left(\phi_{L}+\delta \phi^{c}-\delta \phi_{v}\right)+\right. \\
& \quad\left(I_{g}+\delta I^{c}\right)\left(\omega+\delta \omega^{c}+\dot{\phi}_{L}+\delta \dot{\phi}^{c}\right) \cos \left(\phi_{L}+\delta \phi^{c}-\delta \phi_{v}\right) \\
& \left.\dot{\tilde{I}}_{b} \cos \left(\delta \phi_{v}+\phi^{s}+\delta \phi^{s}\right)-\tilde{I}_{b}\left(\omega+\delta \omega^{c}-\dot{\phi}^{s}-\delta \dot{\phi}^{s}\right) \sin \left(\delta \phi_{v}+\phi^{s}+\delta \phi^{s}\right)\right\}, \\
& \tilde{V}=V+\delta V \\
& \tilde{I}_{b}=I_{b} / K_{g} .
\end{aligned}
$$

Equations 11 and 12 are second order and non-linear. They can be reduced to first-order non-linear form by using the approximations shown below. 


$$
\begin{aligned}
& \ddot{\tilde{V}} \ll 2 \sigma H \dot{\tilde{V}} \\
& \tilde{V} \delta \ddot{\phi}_{v} \ll 2 \sigma H\left(\omega+\delta \omega^{c}\right) \tilde{V} \text { and } 2 \sigma H \tilde{V} \delta \dot{\phi}_{\nu} \\
& \tilde{V} \delta \dot{\phi}_{v}^{2} \ll 2\left(\omega+\delta \omega^{c}\right) \tilde{V} \delta \dot{\phi}_{v} \\
& 2 \dot{\tilde{V}} \delta \dot{\phi}_{v} \ll 2\left(\omega+\delta \omega^{c}\right) \dot{\vec{V}} \\
& \tilde{V}\left(\dot{\omega}+\delta \dot{\omega}^{c}\right) \ll 2 \sigma H\left(\omega+\delta \omega^{c}\right) \tilde{V} .
\end{aligned}
$$

The reduced order non-linear model becomes:

$$
\begin{aligned}
& 2 \sigma H \dot{\hat{V}}-2\left(\omega+\delta \omega^{c}\right) \tilde{V} \delta \dot{\phi}_{v}+\left[\left(\omega_{R}+\delta \omega_{R}\right)^{2}-\left(\omega+\delta \omega^{c}\right)^{2}\right] \tilde{V}=f_{1}, \\
& 2\left(\omega+\delta \omega^{c}\right) \dot{\vec{V}}+2 \sigma H \tilde{V} \delta \dot{\phi}_{v}+2 \sigma H\left(\omega+\delta \omega^{c}\right) \tilde{V}=f_{2} .
\end{aligned}
$$

3.1.2 Cavity Tuning Model. A simplest loop representing a cavity-tuning system is assumed to have a wide-bandwidth bias regulator with an amplifier of $K_{R}$ and supplying a current to the tuning coil. The coil has a single-pole transfer function with a pole at ' $a$ ' rad/sec. If $\phi_{z}^{0}$ is the ideal tuning required by the system, and $\phi_{Z}$ is the actual tuning error measured by the tuning phase detector, then the block diagram shown in Figure 5 represents a simple tuning loop. In the block diagram $i_{\text {tune }}^{c}$ is shown to represent the output current of the bias regulator. The time domain model becomes

$$
\dot{\phi}_{z}=-a \phi_{z}+a K_{R}\left(\phi_{z}^{0}-\phi_{z}\right)
$$

Here we remind to the reader that the tuning loop phase detector is assumed to measure the phase angle between the total current, $\dot{i}_{t}$, and the gap voltage, $V_{\text {ideal }}$, as shown in Figure 3 . Some experts have argued that the tuning loop phase detector actually measures the angle between actual generator current, $i_{\mathrm{g}}$, and voltage $v$ in the vector diagram of Figure 3 , so that it is equal to $\phi_{L}$ plus or minus the phase modulations of generator current and gap voltage. Due to the abrupt termination of the SSC project we were left with no time to investigate this further. Therefore we urge the interested readers to know our assumptions clearly in the model we have derived. The conclusions about loop cross couplings and transfer function plots are as a result of the 
model we have used; These conclusions are believed to be consistent with experimental observation.

\subsection{Global Control Model}

Detailed steps involved in deriving the global model are shown in Reference 8 for low-intensity machines. However, the model will have additional terms when we include cavity dynamics. The synchronization model remains unchanged which is repeated below.

$$
\delta \dot{S}=\left(\dot{v}^{s} / v^{s}\right) \delta S+\left(-\left(v^{s} \eta \gamma_{T}^{2}\right) / R^{s}\right) \delta R-\left(\dot{v}^{s} / v^{s}\right) \delta S_{0}+\frac{v^{s}}{\gamma^{2} B} \delta B
$$

Where $\delta S$ is the synchronization phase error, $\delta R$ is the average radial offset from the central orbit, $\delta B$ is the magnetic field error from the ideal curve $B, R^{s}$ is the radius of the machine, and $v^{s}$ is the velocity of the synchronous particle. While deriving the model representing transverse orbital deviations, the particle phase $\phi$ contains the nominal synchronous phase, phase representing coherent dipole motion, $\delta \phi^{s}$, and the phase shift $\delta \phi_{v}$. The non-linear model is in the form shown below

$$
\delta \dot{R}=-\frac{\dot{A_{1}}}{A_{1}} \delta R+\frac{A_{3}}{A_{1}}\left(1+\frac{\delta V}{V}\right) \sin \left(\delta \phi_{v}+\phi^{s}+\delta \phi^{s}\right)-\frac{A_{3}}{A_{1}} \sin \phi^{s}-\frac{\dot{A_{2}}}{A_{1}} \delta B
$$

Where the coefficients $A_{1}$ and $A_{3}$ are defined later in Table I in this paper. Also the differential equation for the synchrotron phase oscillations is modified by additional terms, which are the derivatives of the phase shift and are shown below.

$$
\delta \dot{\phi}^{s}=\frac{2 \pi f \eta \gamma_{T}^{2}}{R^{s}} \delta R+\delta \omega^{c}-\delta \dot{\phi}_{\nu}-\dot{\phi}^{s}+\frac{2 \pi f}{\gamma^{2} B} \delta B
$$

\subsection{Linear State Space Model}

Since most of the signals are small, we can derive a control model by linearizing Eqs. (15) through (19). While linearizing Eq. (15) the following approximations are used. 


$$
\begin{aligned}
& \cos \left(\delta \phi^{c}-\delta \phi_{v}\right) \approx 1 \\
& \sin \left(\delta \phi^{c}-\delta \phi_{v}\right) \approx \delta \phi^{c}-\delta \phi_{\nu} \\
& \cos \left(\delta \phi^{s}+\delta \phi_{v}\right) \approx 1 \\
& \sin \left(\delta \phi^{s}+\delta \phi_{v}\right)=\delta \phi^{s}+\delta \phi_{v} \\
& \dot{\phi}_{L} \ll \omega \quad \delta \dot{\phi}^{c} \ll \omega \quad \dot{I}_{g} \approx 0 \\
& \dot{\tilde{I}}_{b} \approx 0 \quad \delta \dot{\phi}^{c} \approx 0 \quad \delta \dot{I}^{c} \approx 0 .
\end{aligned}
$$

The products of small quantities such as $\delta \omega^{c} \delta V, \delta V \delta \dot{\phi}_{v}$ etc., are ignored. The tuning angle $\phi_{z}$ is introduced ${ }^{1}$ as below by assuming $\delta \omega_{R} \approx \delta \omega^{c}$

$$
\begin{gathered}
\left(\omega_{R}+\delta \omega_{R}\right)^{2}-\left(\omega+\delta \omega^{c}\right)^{2}=2 \omega \sigma \tan \phi_{z}+2 \sigma \delta \omega^{c} \tan \phi_{z} \\
\tan \phi_{z}=\tan \phi_{z}^{0}+\sec ^{2} \phi_{z}^{0}\left(\phi_{z}-\phi_{z}^{0}\right) .
\end{gathered}
$$

From the vector diagram, under steady-state conditions the following equations hold good.

$$
\begin{array}{ll}
I_{g} \cos \phi_{L}=I_{0}\left(1+Y \sin \phi^{s}\right) & I_{0}=\frac{V H}{R K_{g}} ; \quad Y=\frac{\tilde{I}_{b}}{I_{0}} \\
\tilde{I}_{b} \cos \phi^{s}=I_{g} \sin \phi_{L}+I_{0} \tan \phi_{z}^{0} & \tilde{I}_{b}=\frac{I_{b}}{K_{g}} . \\
\tan \phi_{L}=\frac{Y \cos \phi^{s}-\tan \phi_{z}^{0}}{1+Y \sin \phi^{s}} &
\end{array}
$$

Note that the direct $\mathrm{rf}$ feedback (term $H$ ) reduces the beam loading term, $Y$. After linearizing Eq. (15) and substituting the approximations, we get the state space equations in the form shown in Table I, which follows Appendix B.

\section{MODEL VALIDATION TESTS}

Once the model is constructed for the control system in hand, it is logical to test the model to compare with some known quantities. Pedersen derived a transfer function model for some of the fast loops for a non-time-varying machine. In Appendix B we show the derivation of the 
Pedersen model from the equations shown in Table I as a special case to confirm the validity of the matrices and satisfy Robinson's stability criteria. A more appropriate comparison is to use the longitudinal particle tracking code and compare the states to those obtained by integrating the model. In this way, we would have made the direct comparison of the model for the time-varying conditions. The longitudinal tracking code was developed by us with a lumped cavity which is represented in the form of an equivalent circuit. We show in Reference 10 the description of the tracking code with the cavity model and special measurement techniques to extract the beam phase.

In Figures 6 through 12 the radial position, beam phase error, cavity amplitude error, and cavity phase errors are plotted against time for up to 50 milliseconds with all the loops opened. The solid lines represent the simulated results using the longitudinal tracking code with a discrete time of about 4000 units-per-one rf period. The dashed lines are obtained by using the non-linear global and local models, Eqs. (15) to (19). To create synchrotron oscillations we injected a particle with a $10 \mathrm{MeV}$ injection error from the Linac to the LEB and tracked a single particle up to $50 \mathrm{~ms}$. A cavity with a $\mathrm{Q}$ of 5000 was used. A linear model would not be practical for large amplitude oscillations of over 40 degrees beam phase error, hence the comparison is not shown. In conclusion, the model predicts the essential dynamics of the particle.

The cavity voltage error, $\delta V$, has very high initial transients when there is no feedback as shown in Figure 11 for first $1 \mathrm{~ms}$ after injection. This transient has been identified to be due to the high rate of change of voltage at the beginning, just after injection. In the state-space equation of Table I we have shown terms such as $c_{4}$ and $c_{5}$. They are zero in Pedersen's model due to no direct rf feedback term $(H=1)$ and no time varying terms $(\dot{V}=0)$. The direct rf feedback reduces the initial voltage transient on the gap, but is found to be insufficient. In theory the amplitude and phase loops must be able to reduce the transient to zero, but this is restricted by the overall stability limits and the cavity tuning conditions. Using the state-space model and special compensation techniques we can control the voltage transients to within specifications. Detailed study on this topic is a subject for another paper and will be pursued in 
the future. In the discussion to follow special techniques are shown to investigate the interaction between loops.

\section{COUPLED TRANSFER FUNCTIONS}

The linear state-space model is a good start to study the coupled transfer characteristics. Since our control problem is multi-variable, at first we have to realize a state-space description for a multi-input multi-output case. To proceed, let us consider the model of Table I in terms of system matrix $\underline{A}$ and input matrix $\underline{B}$ as follows:

$$
\underline{\dot{x}}=\underline{A} \underline{x}+\underline{B} \underline{u}+\underline{d},
$$

where $\underline{x}$ represents the states and $\underline{u}$ represents various control signals. The additional term $\underline{d}$ is a disturbance matrix with parameters shown in Table I. The disturbance matrix has important concern with regard to controlling the beam for time-varying machines which will not be discussed in this paper. For studying coupled transfer characteristics we ignore the disturbance matrix.

Consider the output matrix $\underline{y}$ defined such that it satisfies

$$
\underline{y}=\underline{C} \underline{x}
$$

where $\underline{C}$ is an identity matrix. The outputs are defined by the above equation.

Taking Laplace Transtorm of Eqs. (23) and (24), the following equation can be obtained

$$
\begin{aligned}
\underline{Y}(s) & =\underline{C}(s \underline{I}-\underline{A})^{-1} \underline{B} \underline{u} \\
& =\underline{G}(s) \underline{u} .
\end{aligned}
$$

The matrix $\underline{G(s)}$ is an open-loop transfer matrix defined between the control inputs and the outputs. It is given by 


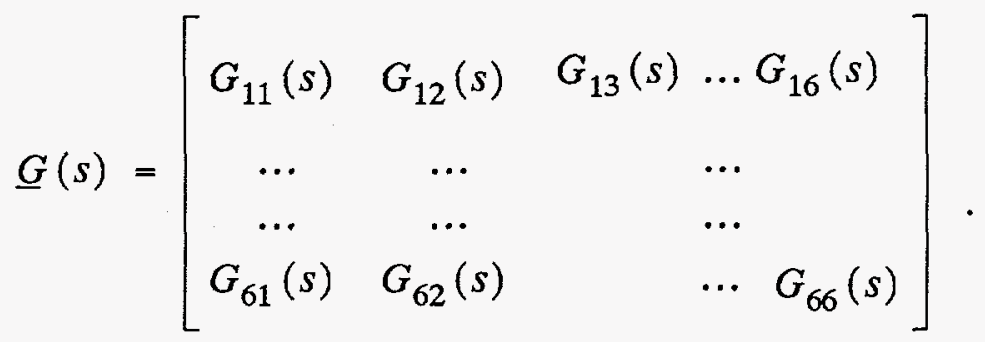

The components of the open loop transfer matrices $\underline{G}(s)$ are shown below in terms of the known measured quantities and the control parameters. They are:

$$
\begin{aligned}
& G_{11}(s)=\frac{X_{1}(s)}{U_{1}(s)}=\frac{\Delta S(s)}{\Delta F^{c}(s)} \quad G_{21}(s)=\frac{X_{2}(s)}{U_{1}(s)}=\frac{\Delta R(s)}{\Delta F^{c}(s)} \\
& G_{12}(s)=\frac{X_{1}(s)}{U_{2}(s)}=\frac{\Delta S(s)}{\Delta F^{c}(s)} \quad G_{22}(s)=\frac{X_{2}(s)}{U_{2}(s)}=\frac{\Delta R(s)}{\Delta F^{c}(s)} \\
& G_{13}(s)=\frac{X_{1}(s)}{U_{3}(s)}=\frac{\Delta S(s)}{\Delta F^{c}(s)} \quad G_{23}(s)=\frac{X_{2}(s)}{U_{3}(s)}=\frac{\Delta R(s)}{\Delta F^{c}(s)} \\
& G_{14}(s)=\frac{X_{1}(s)}{U_{4}(s)}=\frac{\Delta S(s)}{\Delta I^{c}(s)} \quad G_{24}(s)=\frac{X_{2}(s)}{U_{4}(s)}=\frac{\Delta R(s)}{\Delta I^{c}(s)} \\
& G_{15}(s)=\frac{X_{1}(s)}{U_{5}(s)}=\frac{\Delta S(s)}{\Delta \phi^{c}(s)} \quad G_{25}(s)=\frac{X_{2}(s)}{U_{5}(s)}=\frac{\Delta R(s)}{\Delta \phi^{c}(s)} \\
& G_{16}(s)=\frac{X_{1}(s)}{U_{6}(s)}=\frac{\Delta S(s)}{I_{\text {tune }}^{c}(s)} \quad G_{26}(s)=\frac{X_{2}(s)}{U_{6}(s)}=\frac{\Delta R(s)}{I_{\text {tune }}^{c}(s)}
\end{aligned}
$$




$$
\begin{aligned}
& G_{31}(s)=\frac{X_{3}(s)}{U_{1}(s)}=\frac{\Delta \phi^{s}(s)}{\Delta F^{c}(s)} \\
& G_{41}(s)=\frac{X_{4}(s)}{U_{1}(s)}=\frac{\Delta V(s)}{\Delta F^{c}(s)} \\
& G_{32}(s)=\frac{X_{3}(s)}{U_{2}(s)}=\frac{\Delta \phi^{s}(s)}{\Delta F^{c}(s)} \\
& G_{42}(s)=\frac{X_{4}(s)}{U_{2}(s)}=\frac{\Delta V(s)}{\Delta F^{c}(s)} \\
& G_{33}(s)=\frac{X_{3}(s)}{U_{3}(s)}=\frac{\Delta \phi^{s}(s)}{\Delta F^{c}(s)} \\
& G_{43}(s)=\frac{X_{4}(s)}{U_{3}(s)}=\frac{\Delta V(s)}{\Delta F^{c}(s)} \\
& G_{34}(s)=\frac{X_{3}(s)}{U_{4}(s)}=\frac{\Delta \phi^{s}(s)}{\Delta I^{c}(s)} \\
& G_{44}(s)=\frac{X_{4}(s)}{U_{4}(s)}=\frac{\Delta V(s)}{\Delta I^{c}(s)} \\
& G_{35}(s)=\frac{X_{3}(s)}{U_{5}(s)}=\frac{\Delta \phi^{s}(s)}{\Delta \phi^{c}(s)} \\
& G_{45}(s)=\frac{X_{4}(s)}{U_{5}(s)}=\frac{\Delta V(s)}{\Delta \phi^{c}(s)} \\
& G_{36}(s)=\frac{X_{3}(s)}{U_{6}(s)}=\frac{\Delta \phi^{s}(s)}{I_{\text {tune }}^{c}(s)} \\
& G_{46}(s)=\frac{X_{4}(s)}{U_{6}(s)}=\frac{\Delta V(s)}{I_{\text {tune }}^{c}(s)} \\
& G_{51}(s)=\frac{X_{5}(s)}{U_{1}(s)}=\frac{\Delta \phi_{v}(s)}{\Delta F^{c}(s)} \\
& G_{61}(s)=\frac{X_{6}(s)}{U_{1}(s)}=0 \\
& G_{52}(s)=\frac{X_{5}(s)}{U_{2}(s)}=\frac{\Delta \phi_{v}(s)}{\Delta F^{c}(s)} \\
& G_{62}(s)=\frac{X_{6}(s)}{U_{2}(s)}=0 \\
& G_{53}(s)=\frac{X_{5}(s)}{U_{3}(s)}=\frac{\Delta \phi_{v}(s)}{\Delta F^{c}(s)} \\
& G_{63}(s)=\frac{X_{6}(s)}{U_{3}(s)}=0 \\
& G_{54}(s)=\frac{X_{5}(s)}{U_{4}(s)}=\frac{\Delta \phi_{v}(s)}{\Delta r^{c}(s)} \\
& G_{64}(s)=\frac{X_{6}(s)}{U_{4}(s)}=0 \\
& G_{55}(s)=\frac{X_{5}(s)}{U_{5}(s)}=\frac{\Delta \phi_{v}(s)}{\Delta \phi^{c}(s)} \\
& G_{65}(s)=\frac{X_{6}(s)}{U_{5}(s)}=0 \\
& G_{56}(s)=\frac{X_{5}(s)}{U_{6}(s)}=\frac{\Delta \phi_{v}(s)}{I_{\text {tune }}^{c}(s)} \\
& G_{66}(s)=\frac{X_{6}(s)}{U_{6}(s)}=\frac{\Delta \phi_{z}(s)}{I_{\text {tune }}^{c}(s)} \text {. }
\end{aligned}
$$


Gain curves of Eq. (26) show the coupling between different inputs and outputs. For example, to study the effect on beam phase, $\left(y_{3}=x_{3}\right)$, due to the simultaneous modulations on (1) the frequency, $\delta f^{c}=u_{3}$, (2) the amplitude of the rf signal, $\delta I^{c}=u_{4}$, and (3) the phase of the rf signal, $\delta \phi^{c}=u_{5}$, we plot gains $G_{33}(s), G_{34}(s)$ and $G_{35}(s)$ with respect to frequency. However, to compare the amplitude of the gains, first they have to be normalized to a certain index. We show below how to normalize the gain matrix. After this, the actual plots of the gains are shown with respect to the modulating frequency for different control signals.

To normalize the open-loop transfer matrix, we first normalize the states and the controls. The normalized state matrix is given by

$$
\begin{array}{rlr}
\underline{\tilde{x}} & =\operatorname{diag}\left\{1 / \underline{x}_{i}^{m}\right\} \underline{x} \\
& =\underline{T}_{x} \underline{x} & \mathrm{i}=1,2, \ldots, 6,
\end{array}
$$

where $\underline{T}_{x}$ is the diagonal transformation matrix for unnormalized states. The elements $x_{i}^{m}$ are the maximum values of the measured states in the state matrix, $\underline{x}$. Similarly, the normalized control matrix is given by

$$
\begin{array}{rlr}
\underline{\tilde{u}} & =\operatorname{diag}\left\{1 / \underline{u}_{i}^{m}\right\} \underline{u} \\
& =\underline{T}_{u} \underline{u} &
\end{array}
$$

where $\underline{T}_{u}$ is the diagonal transformation matrix for unnormalized control quantities. The elements $u_{i}^{m}$ are the maximum values of the control quantities. After substituting Eqs. (27) and (28) in Eqs. (23) and (24) and converting the resulting equation to frequency domain we get a new equation shown below

$$
\begin{aligned}
\tilde{Y}(s) & =\tilde{C}(s \underline{I}-\underline{\tilde{A}})^{-1} \underline{\tilde{B}} \tilde{U}(s) \\
& =\tilde{G}(s) \underline{\tilde{U}}(s),
\end{aligned}
$$

where

$$
\underline{\tilde{A}}=\underline{T}_{x} \underline{A} \underline{T}_{x}^{-1} \quad \underline{\tilde{B}}=\underline{T}_{x}^{-1} \underline{B} \underline{T}_{u}^{-1} \quad \underline{\tilde{C}}=\underline{C} \underline{T}_{x}^{-1}
$$

The normalized gain matrix, $\underline{\tilde{G}}(s)$, is now dimensionless. 
In Table II the maximum values of the states and the control quantities used for the LEB while plotting the gain matrix are shown. Figures 13 through 18 are plotted using the software MATLAB ${ }^{11}$ to show the normalized gains $G_{11}$ to $G_{66}$ of Eq. (26) with respect to frequency at $1 \mathrm{~ms}, 10 \mathrm{~ms}, 20 \mathrm{~ms}$ and $45 \mathrm{~ms}$ from injection. These curves were plotted without the presence of direct $r$ feedback for $Q=5000$ and an average beam current of $100 \mathrm{~mA}$. They are shown to identify the coupling effects between loops at different times during the acceleration cycle. Figures 19 through 24 show the gain variations with frequency at a fixed time of $10 \mathrm{~ms}$ from injection for different values of the direct rf feedback strength, $H$. We selected $H=1$ (no direct If feedback), $H=10,20$, and 30 since for the LEB a feedback strength of 20 has been considered. In these plots the average beam current is equal to $100 \mathrm{~mA}$. With varying rf feedback strength, the plots show the loop interactions due to varying the effective $Q$ of the cavities. Also, to understand the coupling effects due to beam loading strength the gain curves are shown in Figures 25 through 30 with respect to frequency for $H=20$ and varying beam current, $I_{b}=0,1 \mathrm{~A}, 2 \mathrm{~A}, 3 \mathrm{~A}$. Evidently, for no beam current the plots show that the gains associated directly with the beam are zero. All the plots are shown to the same $x$ and $y$-axes scales so that they can be compared easily. At first we show an example to identify the coupling and later summarize the observations made on each figure.

\subsection{Identifying Coupling Between Loops}

In general loops are said to be coupled if the normalized open loop gains are same when measured with a modulation on different control inputs. To understand this, let us concentrate on the beam phase loop alone by considering Figure 15 at $1 \mathrm{~ms}$. There are three curves shown in this figure. The curve with solid line represents the open-loop gain of the beam phase loop. This means the gain of the beam phase loop when measured as follows. Open all the loops including the beam phase loop. (Of course, this condition is impossible to achieve when there is beam in the machine.) Apply frequency modulation of say $5 \mathrm{kHz}$ amplitude to the frequency ramp curve at $10-\mathrm{Hz}$ to $10^{5}-\mathrm{kHz}$ modulation frequency. Each time the modulation frequency is varied to measure the amplitude of the modulation on the synchrotron phase oscillations. By 
taking the ratio of the phase oscillation amplitude and the amplitude of the modulating frequency and then normalizing by using the values shown in Table $\Pi$ a gain curve $\left(G_{33}\right)$ shown by solid line can be obtained. As was anticipated, the curve has a peak at the synchrotron frequency. Since the beam phase can also be affected by the amplitude and phase control on the generator current $\left(\delta I^{c}\right.$ and $\delta \phi^{c}$ ) we need to plot the open loop gain curves for such cross coupling. This can be done by opening all the loops and making a measurement on the beam phase by applying a sine wave modulation on the amplitude and phase controls independently between $10 \mathrm{~Hz}$ to $10^{5} \mathrm{kHz}$. The dashed line represents the open loop gain response $\left(G_{34}\right)$ between the beam phase and the amplitude of the generator current. Similarly, the dash-dotted line represents the open loop gain response $\left(G_{35}\right)$ between the beam phase and the phase of the generator current.

The gain curve, $G_{35}$, in Figure 15 at $1 \mathrm{~ms}$ is following the same amplitude as the gain curve, $G_{33}$, after $700 \mathrm{~Hz}$ up to $100 \mathrm{kHz}$ with a peak at the synchrotron frequency. This means, between $700 \mathrm{~Hz}$ and $100 \mathrm{kHz}$, the control from the beam phase loop is coupled to the local phase loop. To make the beam phase loop operate independently of the local phase loop, they have to be decoupled at the operating region of the beam phase loop; otherwise, they may interact one another and ultimately cause unknown instabilities in the beam. Similarly the gain curve, $G_{34}$, intersects with the gain curve, $G_{33}$, at approximately $10 \mathrm{kHz}$, meaning all three loops are strongly coupled. Also, the figure (Figure 15 at $1 \mathrm{~ms}$ ) shows the generator current amplitude control has a stronger effect on the amplitude of the beam phase oscillations at frequencies below $10 \mathrm{kHz}$ when compared to the amplitude of the frequency control in the beam phase loop. Later in this section we show that a reduction in the $Q$ of the cavity reduces the coupling between the local amplitude loop and the beam phase loop. Whereas, the local phase loop remains coupled to the beam phase loop even after reducing the cavity $\mathrm{Q}$.

Figures 13 through 30 show that all the loops are coupled at the synchrotron frequency. At frequencies below the synchrotron frequency we can identify coupling by taking a closer look at individual gain plots. We present a brief summary of this below. 
Figures 13 and 14: Synchronization and radial loops are not coupled to local amplitude and phase loop controls below synchrotron frequencies. However, the synchronization loop has a dominant effect on the radial position. Hence, to control the radial position when the synchronization loop is closed from the time of injection in the LEB, a 'dead zone' (programmable gain with radial position) may be needed in the radial loop controller. With the dead zone, the radius can be allowed to move freely up to few millimeters around the central orbit for synchronization. Figure 13 at 45 ms suggests an open-loop bandwidth of about $200 \mathrm{~Hz}$ which corresponds to a time constant of $5 \mathrm{~ms}$. Therefore, if the synchronization loop is closed at $45 \mathrm{~ms}$, we have to achieve synchronization in less than $5 \mathrm{~ms}$ which demands a high gain in this loop and consequently only a low or no gain in the radial loop could eliminate the loop interactions.

Figures 13 and 15: The beam phase loop is coupled to the local phase loop in its operating region. Also, by comparing the solid lines in Figures 13 and 15 at $45 \mathrm{~ms}$ we see that the open-loop gain of the beam phase loop matches with that of the synchronization loop at $60 \mathrm{~Hz}$. From $60 \mathrm{~Hz}$ to $200 \mathrm{~Hz}$ the beam phase loop gain increases steeply and that of the synchronization loop decreases to below unity. It means the coupling between the synchronization loop and the beam phase loop is strong in this frequency band. If the synchronization loop is arranged to operate after $45 \mathrm{~ms}$, since it inevitably requires a bandwidth of greater than $200 \mathrm{~Hz}$ to reach synchronism within $5 \mathrm{~ms}$ thereafter, the loop will interact with the beam phase loop. Hence, by including a low-pass filter with a cutoff below $100 \mathrm{~Hz}$ in the synchronization loop and by closing the loop much earlier than $45 \mathrm{~ms}$, interactions with beam phase loop can be greatly reduced. Alternatively, if the specifications on the maximum synchrotron phase oscillations are relaxed to 6 degrees instead of 3 degrees as in Table II, the time required for synchronization can be reduced.

Figure 16: The global frequency control and the local phase loop control do not affect the local amplitude loop except around synchrotron frequencies. The local amplitude loop has an open loop bandwidth close to $100 \mathrm{kHz}$. 
Figure 17: The global frequency control and the local amplitude loop control do not affect the local phase loop except at synchrotron frequencies. The local phase loop has an open loop bandwidth close to $100 \mathrm{kHz}$.

Figure 18 and 24: The tuning control, $I_{\text {tune, }}^{c}$ is affecting all the states including the synchronization phase. These figures show that the cavity tuning system has to be good for the LEB to work since all the loops are coupled to the tuning control.

Figure 19: With the increase in the direct rf feedback, $H>1$, the gain of the synchronization loop with respect to generator current amplitude control is reduced.

Figure 20: With the increase in the direct rf feedback, $H>1$, the gain of the radial loop with respect to generator current amplitude control is reduced.

Figure 21: The beam phase loop is decoupled with the generator current amplitude control for $H=10,20$ and 30 in its operating region around the synchrotron frequency. However, the beam phase loop is not decoupled from the local phase loop control. One of the ways to decouple them is by introducing a tracking band-stop filter in the local phase loop or operate it at frequencies above the synchrotron frequency.

Figure 22 and 23: No significant change in the local amplitude and phase loops in the presence of direct $\mathrm{rf}$ feedback.

Figures 25, 26 and 27: Increase in beam current is not significantly affecting the coupling of the global loops with the local amplitude and phase loops.

Figure 28: The local amplitude loop is coupled strongly to the local phase loop control with increase in beam loading.

Figure 29: The local amplitude control is coupled strongly to the local phase loop with increase in beam loading.

Figure 30 : The tuning control, $I_{\text {time }}^{c}$, is affecting all the states very strongly. 


\section{$7 \quad$ CONCLUSIONS}

A scientific study of the low-level rf feedback loops with rf cavity and beam dynamics for the SSC Low Energy Booster showed clearly the interaction between all the loops. We see that the fast and slow feedback loops are coupled at synchrotron frequencies. The synchronization and radial loops are also coupled to one another but do not interact with the local amplitude and phase control loops below synchrotron frequencies. Whereas the beam phase loop and the local phase loop are coupled during the complete operating region of the beam phase loop. The interactions between them are stronger even with a reduced $\mathrm{Q}$ of the cavity. One way to decouple them is by operating the local phase loop above synchrotron frequencies or have a tracking band-stop filter in the local phase loop. With a high Q cavity and increased beam current the analysis showed that the local amplitude loop also has increased coupling to the beam phase loop. Furthermore, the cavity tuning system interacts strongly with all the loops. Hence, the tuning system must be good for other machine parameters to be under control.

The normalized open-loop gain plots drawn with respect to frequency for different control inputs are useful to know the available bandwidth on each loop and then design appropriate filter corner frequencies to decouple them. The method used in this paper is especially more useful for fast-cycling synchrotrons. The decoupling can also be done by following some of the techniques outlined in Reference 12 using the time varying state-space model derived in this paper. Intuitively, the multivariable state-space control method should give better results since all the loops are considered together while designing decoupling compensators. The results of the systematic study on the decouplers will be reported in future.

\section{ACKNOWLEDGEMENTS}

The authors would like to thank Bob Webber for his continued encouragement to carry on the work discussed in this paper. Also, the assistance given by G. Rees, Tai-Sen Wang and N.K. Mahale in various technical discussions while developing the control model is gratefully acknowledged. 


\section{REFERENCES}

1. F. Pedersen, "Beam Loading Effects in the CERN PS Booster," IEEE Transactions on Nuclear Science, Vol. NS-22, No. 3, June 1975.

2. Tai-Sen F. Wang, "Bunched-Beam Longitudinal Mode-Coupling and Robinson-Type Instabilities," Particle Accelerators, Vol. 34, PP. 105-126, 1990.

3. S.R. Koscielniak and Tai-Sen F. Wang, "Compensation of RF Transients During Injection into the Collector Ring of the TRIUMF KAON Factory," Particle Accelerator Conference, San-Francisco, May 6-9, 1991.

4. F. Pedersen, "A Novel RF Cavity Tuning Feedback Scheme for Heavy Beam Loading," IEEE Transactions on Nuclear Science, Vol. NS-32, No. 5, October 1985.

5. R. Johnson, S. Van der Meer, F. Pedersen and G. Shering, "Computer Control of RF-Manipulations in the CERN Antiproton Accumulator," IEEE Transactions on Nuclear Science, Vol. NS-30, No. 4, August 1983.

6. J.M. Baillod, L. Magnani, G. Nassibian, F. Pedersen and W. Weissflog, "A Second Harmonic (6-16 MHz) RF System with Feedback-Reduced Gap Impedance for Accelerating Flat-topped bunches in the CERN PS Booster," IEEE Transactions on Nuclear Science, Vol. NS-30, No. 4, August 1983.

7. D. Boussard, "Control of Cavities with High Beam Loading," IEEE Transactions on Nuclear Science, Vol. NS-32, No. 5, October 1985.

8. L.K. Mestha, C.M. Kwan, and K.S. Yeung, "Instabilities in Beam Control Feedback Loops in Proton Synchrotrons," Accepted for publication in the Journal of Particle Accelerators.

9. D. Boussard and G. Lambert, "Reduction of the Apparent Impedance of Wide Band Accelerating Cavities by RF Feedback," IEEE Transactions on Nuclear Science, Vol NS-30, No. 4, August 1983. 
10. C.M. Kwan and L.K. Mestha, "Simulation of Longitudinal Beam Dynamics With the Inclusion of RF Cavity," SSCL-617, March 1993.

11. MATLAB Reference Guide, The Math Works Inc., Natick, Mass. 01760, USA, August 1992.

12. J.M. Maciejowski, Multivariable Feedback Design, Addison-Wesley Publishing Company, 1989.

\section{APPENDIX A}

\section{A.1 Condition for Time-Varying Approach}

Solution of the second-order differential equation of the cavity for an appropriate generator current will result into the gap voltage. For machines such as the LEB, the generator current is driven by a variable frequency oscillator. For it to work, the gap voltage, $v$, must track the voltage applied on the anode of the power amplifier. It is usual practice in the accelerator field to represent the gap voltage and generator current as follows

$$
\begin{aligned}
& v=V e^{j \omega t} \\
& i_{g}=I_{g} e^{j \omega t} .
\end{aligned}
$$

By substituting $I_{g}=\frac{V_{\text {input }}}{R}, \sigma=\frac{\omega_{R}}{2 Q}$, holding cavities on tune $\left(\omega_{R}=\omega\right)$ and sweeping the frequency $\omega$ between $2 \pi \times 47 \mathrm{MHz}$ to $2 \pi \times 59.9 \mathrm{MHz}$, we must in principle obtain the gap voltage $v$ shown by Eq. (A1). Our simulation studies showed that the voltage was unable to track $V_{\text {input }}$ as shown by curve with dashed line in Figure A.1. On the other hand if the generator current was represented as

$$
i_{g}=I_{g} e^{j \int \omega d t},
$$

there was good tracking as shown by curve with solid lines in Figure A.1. The ideal input gap voltage $V_{\text {input }}$, hidden in curve shown with solid line. The ideal curve has none of the small oscillations on the curve with solid lines. Small oscillations are due to the synchrotron 
frequency. With a reduced rate of change of the accelerating frequency, $\omega$, the tracking of the voltage was improved. This means the modeling approach shown in Eq. (A1) is incorrect for machines like the LEB. Since the LEB frequency is time varying we need to have a condition to know when exactly we can cross the boundaries between time varying and time invariant approaches. Some simple equations are derived below for the cavity parameter and accelerating frequency.

Substituting equations for $v, \dot{v}$ and $\ddot{v}$ into Eq. (2), we obtain

$$
\begin{gathered}
{\left[\ddot{V}+\dot{V}[2 \sigma+j 2(\omega+\dot{\omega} t)]+V\left[\omega_{R}^{2}-(\omega+\dot{\omega} t)^{2}+j\{(2 \dot{\omega}+\ddot{\omega} t)+2 \sigma(\omega+\dot{\omega} t)\}\right] e^{j \omega t}\right]} \\
=2 \sigma R \dot{i}_{t} .
\end{gathered}
$$

The right-hand side of Eq. (A3) can be written as,

$$
u=U e^{j \omega t} .
$$

Since $V$ and $U$ can be represented as phasors, we can write them as real and imaginary parts as follows:

$$
\begin{aligned}
V & =V_{R}+j V_{I} \\
U & =U_{R}+j U_{I} .
\end{aligned}
$$

By substituting Eqs. (A4) and (A5) into (A3) and comparing the real and imaginary parts, the following equation can be obtained in state space form

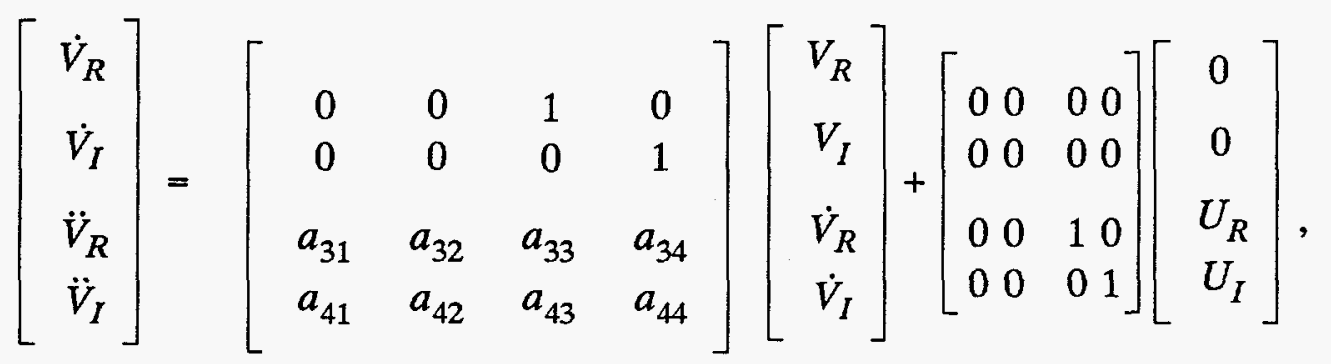


where

$$
\begin{array}{llll}
a_{31}=-\omega_{R}^{2}+(\omega+\dot{\omega} t)^{2} & a_{33}=-2 \sigma & a_{41}=-a_{32} & a_{43}=-a_{34} \\
a_{32}=(2 \dot{\omega}+\ddot{\omega} t)+2 \sigma(\omega+\dot{\omega} t) & a_{34}=2(\omega+\dot{\omega} t) & a_{42}=-a_{31} & a_{44}=a_{33} .
\end{array}
$$

Equation (A6) is in the standard form

$$
\underline{\dot{x}}=\underline{A} \underline{x}+\underline{B} \underline{u} .
$$

If we assume that we have frozen the time then, $\operatorname{det}(s \underline{I}-\underline{A})=0$ is the characteristic equation. It turns out that the characteristic equation is 4 th order.

$$
s^{4}+a_{3} s^{3}+a_{2} s^{2}+a_{1} s+a_{0}=0
$$

where the coefficients of Eq. (A8) are given by

$$
\begin{aligned}
a_{3}= & 4 \sigma \\
a_{2}= & 4 \sigma^{2}+4(\omega+\dot{\omega} t)^{2}+2\left\{\omega_{R}^{2}-(\omega+\dot{\omega} t)^{2}\right\} \\
a_{1}= & 4 \sigma(\omega+\dot{\omega} t)^{2}+2(\omega+\dot{\omega} t)(2 \dot{\omega}+\ddot{\omega} t)+ \\
& 4 \sigma\left\{\omega_{R}^{2}-(\omega+\dot{\omega} t)^{2}\right\}+2(\omega+\dot{\omega} t)\{2 \dot{\omega}+\dddot{\omega} t+2 \sigma(\omega+\dot{\omega} t)\} \\
a_{0}= & {\left[\left\{\omega_{R}^{2}-(\omega+\dot{\omega} t)^{2}\right\}^{2}+\{2 \dot{\omega}+\dddot{\omega} t+2 \sigma(\omega+\dot{\omega} t)\}\right]^{2} . }
\end{aligned}
$$

When $\dot{\omega}=0$, the coefficients are independent of time. For simplicity we can assume that the cavities are on tune, i.e., $\omega_{R}^{2}=\omega^{2}$. Now by comparing the coefficients of Eq. (A9) with those for $\dot{\omega}=0$ we can obtain the following inequalities.

$$
\begin{gathered}
\frac{\dot{\omega} t}{\omega} \ll 1 \\
\frac{\sigma}{\omega} » \frac{1}{2} \frac{\dddot{\omega} t}{\omega^{2}}+\frac{\dot{\omega}}{\omega^{2}}
\end{gathered}
$$




$$
\frac{\sigma}{\omega} \gg \frac{\dot{\omega} t}{\omega}
$$

If the inequalities presented above are satisfied, then the mathematical formulation shown in Eq. (A1) is close to being a time invariant system. Hence, the loop models constructed based on such an approach are accurate, even for fast-cycling machines. However, for the design parameters of the LEB, inequality in Eq. (A12) is violated. Hence the representation shown by Eq. (A1) does not give accurate model. We, therefore, have to consider a more general method as in Eq. (A2).

\section{APPENDIX B}

\section{B.1 Validity of Linear Model with Pedersen Model}

The linear model shown in state space form of Table I can be compared to Pedersen's model ${ }^{1}$ by disabling the direct rf feedback $(H=1)$, the frequency control $\left(\delta \omega^{c}=0\right)$, and allowing no time variation to the cavity and beam parameters. Using the linearized version of Eq. (15) and the tuning angle Eq. (21) and steady state Eq. (22) we get

$$
\begin{aligned}
& \sigma \dot{a}_{v}-\omega \delta \dot{\phi}_{v}+\sigma \omega \tan \left(\phi_{z}\right)^{0} a_{v}= \\
& \frac{\sigma \omega}{I_{0}}\left\{\left(I_{g} \cos \phi_{L}-I_{b} \sin \phi^{s}\right) \delta \phi_{v}-I_{g} \cos \phi_{L} \delta \phi^{c}-I_{b} \sin \phi^{s} \delta \phi^{s}-\sin \phi_{L} \delta I^{c}\right\}, \\
& \omega \dot{a}_{v}+2 \sigma \delta \dot{\phi}_{v}+2 \sigma \omega a_{v}= \\
& \frac{\sigma \omega}{I_{0}}\left\{\left(I_{g} \sin \phi_{L}-I_{b} \cos \phi^{s}\right) \delta \dot{\phi}_{v}-I_{g} \sin \phi_{L} \delta \phi^{c}-I_{b} \cos \phi^{s} \delta \phi^{s}-\cos \phi_{L} \delta I^{c}\right\},
\end{aligned}
$$

where $a_{v}=\frac{\delta V}{V}$

While deriving the above equation we have assumed $K_{g}=1$. Now by taking Laplace Transform of Eqs. (B1) and (B2) and by assuming the Laplace parameter $s \ll \omega \tan \left(\phi_{Z}\right)$ as in Reference 1, we obtain 


$$
\begin{gathered}
G_{p a}^{b}(s)=\frac{A_{v}(s)}{\delta \Phi^{s}(s)}=-Y\left[\frac{\sigma^{2}\left(\tan \phi_{z}^{0} \sin \phi^{s}+\cos \phi^{s}\right)-\sigma s \cos \phi^{s}}{s^{2}+2 \sigma s+\sigma^{2}\left(1+\tan ^{2} \phi_{z}^{0}\right)}\right] \\
G_{p p}^{b}(s)=\frac{\delta \Phi_{v}(s)}{\delta \Phi^{s}(s)}=-Y\left[\frac{\sigma^{2}\left(\tan \phi_{z}^{0} \cos \phi^{s}+\sin \phi^{s}\right)+\sigma s \sin \phi^{s}}{s^{2}+2 \sigma s+\sigma^{2}\left(1+\tan ^{2} \phi_{z}^{0}\right)}\right] \\
G_{p a}^{g}(s)=\frac{A_{v}(s)}{\delta \Phi^{c}(s)}=\frac{-\sigma^{2} y\left(\tan \phi_{z}^{0} \sin \phi^{s}+\cos \phi^{s}\right)+\sigma s\left(\tan \phi_{z}^{0}-Y \cos \phi^{s}\right)}{s^{2}+2 \sigma s+\sigma^{2}\left(1+\tan \phi_{z}^{0}\right)} \\
G_{a a}^{g}(s)=\frac{A_{v}(s)}{\delta I^{c}(s) / I_{g}}=\frac{\sigma^{2} \tan \phi_{z}^{0}\left(\tan \phi_{z}^{0}-Y \cos \phi^{s}\right)+\sigma(s+\sigma)\left(1+Y \sin \phi^{s}\right)}{s^{2}+2 \sigma s+\sigma^{2}\left(1+\tan ^{2} \phi_{z}^{0}\right)} \\
=G_{p p}^{g}(s)=\frac{\delta \Phi_{v}(s)}{\delta \Phi^{c}(s)} \\
G_{a p}^{g}(s)=\frac{\delta \Phi_{v}(s)}{\delta I^{c}(s) / I_{g}}=-G_{p a}^{g}(s) .
\end{gathered}
$$

The sign of the transfer function connected with beam in Eq. (B3) is different since the convention of $\delta \phi^{S}$ used in Eq. (B3) is arbitrary.

To derive the coupled transfer function with the tuning system, we rewrite Eq. (21) as

$$
\left(\omega_{R}+\delta \omega_{R}\right)^{2}-\omega^{2}=2 \omega \sigma \tan \phi_{z}^{0}+2 \omega_{R} x
$$

where $x=\delta \omega_{R}$, a shift in resonant frequency.

Using Eq. (B4) and Eq. (22) in the linearized version of Eq. (15), and by ignoring the terms associated with $\delta \phi^{c}$ and $\delta \phi^{S}$ we get 


$$
\begin{gathered}
\sigma \dot{a}_{v}-\omega \delta \dot{\phi}_{v}+\sigma \omega \tan \phi_{z}^{0} a_{v}=\sigma \omega \delta \phi_{v}-\omega_{R} x \\
\omega \dot{a}_{v}+\sigma \delta \dot{\phi}_{v}+\sigma \omega a_{v}=-\sigma \omega \tan \phi_{z}^{0} \delta \phi_{v} .
\end{gathered}
$$

Taking Laplace Transform of Eq. (B5) and assuming $s \ll \omega \tan \left(\phi_{Z}\right)$ the following transfer function models are derived

$$
\begin{gathered}
G_{x a}(s)=\frac{A_{v}(s)}{X(s)}=\frac{-\sigma \tan \phi_{z}^{0}}{s^{2}+2 \sigma s+\sigma^{2}\left(1+\tan ^{2} \phi_{z}^{0}\right)} \\
G_{x p}(s)=\frac{\delta \Phi_{v}(s)}{X(s)}=\frac{s+\sigma}{s^{2}+2 \sigma s+\sigma^{2}\left(1+\tan ^{2} \phi_{z}^{0}\right)} .
\end{gathered}
$$

This shows our state space model meets the Robinson stability criteria for a time invariant case. 
TABLE I: A linear state-space control model.

$$
\left[\begin{array}{c}
\dot{x}_{1} \\
\dot{x}_{2} \\
\dot{x_{3}} \\
\dot{x}_{4} \\
\dot{x}_{5} \\
\dot{x}_{6}
\end{array}\right]=\left[\begin{array}{cccccc}
a_{11} & a_{12} & 0 & 0 & 0 & 0 \\
0 & a_{22} & a_{23} & a_{24} & a_{23} & 0 \\
0 & a_{32} & a_{33} & a_{34} & a_{35} & a_{36} \\
0 & a_{42} & a_{43} & a_{44} & a_{45} & a_{46} \\
0 & a_{52} & a_{53} & a_{54} & a_{55} & a_{56} \\
0 & 0 & 0 & 0 & 0 & a_{66}
\end{array}\right]\left[\begin{array}{c}
x_{1} \\
x_{2} \\
x_{3} \\
x_{4} \\
x_{5} \\
x_{6}
\end{array}\right]
$$$$
+\left[\begin{array}{cccccc}
0 & 0 & 0 & 0 & 0 & 0 \\
0 & 0 & 0 & 0 & 0 & 0 \\
0 & 0 & b_{33} & b_{34} & b_{35} & 0 \\
0 & 0 & b_{43} & b_{44} & b_{45} & 0 \\
0 & 0 & b_{53} & b_{54} & b_{55} & 0 \\
0 & 0 & 0 & 0 & 0 & b_{66}
\end{array}\right]\left[\begin{array}{c}
0 \\
0 \\
u_{3} \\
u_{4} \\
u_{5} \\
u_{6}
\end{array}\right]+\left[\begin{array}{c}
d_{11} \delta B \\
d_{21} \delta B \\
-\dot{\phi}^{s}+d_{31} \delta B \\
c_{4}+d_{41} \delta B \\
c_{5}+d_{51} \delta B \\
c_{6}
\end{array}\right]
$$ 
TABLE I: A linear state-space control model. (Continued)

$$
\begin{aligned}
& \underline{\dot{x}}=\underline{A} \underline{x}+\underline{B} \underline{u}+\underline{d} \\
& x_{1}=\delta S \quad x_{5}=\delta \phi_{v} \quad u_{1}=u_{2}=u_{3}=\delta \omega^{c} \\
& x_{2}=\delta R \quad x_{6}=\phi_{z}-\phi_{z}^{0} \quad u_{4}=\delta I^{c} \\
& x_{3}=\delta \phi^{s} \quad=\delta \phi_{z} \quad u_{5}=\delta \phi^{c} \\
& x_{4}=\delta V \quad u_{6}=i_{\text {tune }}^{c}\left(=-K_{R} x_{6} \text { when the loop is closed }\right) \\
& a_{11}=v^{s} / v^{s} \\
& a_{12}=-\left(\nu^{s} \eta \gamma_{T}^{2}\right) / R^{s} \\
& a_{23}=\frac{A_{3}}{A_{1}} \cos \phi^{s} \\
& a_{22}=-\dot{A_{1}} / A_{1} \\
& a_{24}=\frac{A_{3}}{V A_{1}} \sin \phi^{s} \\
& a_{32}=\frac{2 \pi f \eta \gamma_{T}^{2}}{R^{s}}\left[1-\sigma R K_{g} \tilde{I}_{b}\left\{-F_{21} \cos \phi^{s}+F_{22} \sin \phi^{s}\right\}\right] \\
& a_{33}=\sigma R K_{g} \tilde{I}_{b} \omega\left[F_{21} \sin \phi^{s}+F_{22} \cos \phi^{s}\right] \\
& a_{34}=\sigma \omega\left[F_{21} \tan \phi_{z}^{0}+F_{22} H\right] \\
& a_{35}=-\sigma R K_{g} \omega\left[F_{21}\left(I_{g} \cos \phi_{L}-\tilde{I}_{b} \sin \phi^{s}+\dot{I}_{g} \sin \phi_{L}\right)+F_{22}\left(I_{g} \sin \phi_{L}-\tilde{I}_{b} \cos \phi^{s}-\dot{I}_{g} \cos \phi_{L}\right)\right] \\
& a_{36}=F_{21} \sigma \omega V \sec ^{2} \phi_{z}^{0} \\
& b_{33}=1+F_{21}\left(\sigma V \tan \phi_{z}^{0}+\sigma R K_{g} I_{g} \sin \phi_{L}\right)-F_{22}\left(\sigma R K_{g} I_{g} \cos \phi_{L}-\dot{V}-\sigma V H\right) \\
& b_{34}=\sigma R K_{g} I_{g}\left(F_{21} \sin \phi_{L}-F_{22} \cos \phi_{L}\right) \\
& b_{35}=\sigma R K_{g}\left\{F_{21}\left(I_{g} \omega \cos \phi_{L}+\dot{I}_{g} \sin \phi_{L}\right)-F_{22}\left(\dot{I}_{g} \cos \phi_{L}-I_{g} \omega \sin \phi_{L}\right)\right\} \\
& a_{42}=\frac{2 \pi f \eta \gamma^{2}}{R^{s}} \sigma R K_{g} \tilde{I}_{b}\left[-F_{11} \cos \phi^{s}+F_{12} \sin \phi^{s}\right] \\
& a_{43}=-\sigma R K_{g} \tilde{I}_{b} \omega\left[F_{11} \sin \phi^{s}+F_{12} \cos \phi^{s}\right]
\end{aligned}
$$


TABLE I: A linear state-space control model. (Continued)

$$
\begin{aligned}
& a_{44}=-\sigma \omega\left[F_{11} \tan \phi_{z}^{0}+F_{12} H\right] \\
& a_{45}=\sigma R K_{g}\left\{I_{g} \omega\left(F_{11} \cos \phi_{L}+F_{12} \sin \phi_{L}\right)-\tilde{I}_{b}\left(F_{11} \sin \phi^{s}+F_{12} \cos \phi^{s}\right)+\right. \\
& \left.\left(F_{11} \dot{I}_{g} \sin \phi_{L}-F_{12} \dot{I}_{g} \cos \phi_{L}\right)\right\} \\
& a_{46}=-F_{11} \sigma \omega V \sec ^{2} \phi_{z}^{0} \\
& b_{43}=-\sigma V F_{11} \tan \phi_{z}^{0}+\sigma R K_{g} I_{g}\left(-F_{11} \sin \phi_{L}+F_{12} \cos \phi_{L}\right)-F_{12}(\dot{V}+\sigma V H) \\
& b_{44}=\sigma R K_{g} \omega\left[-F_{11} \sin \phi_{L}+F_{12} \cos \phi_{L}\right] \\
& b_{45}=-\sigma R K_{g}\left[I_{g} \omega\left(F_{11} \cos \phi_{L}+F_{12} \sin \phi_{L}\right)+\dot{I}_{g}\left(F_{11} \sin \phi_{L}+F_{12} \cos \phi_{L}\right)\right] \\
& a_{52}=\frac{2 \pi f \eta \gamma_{T}^{2}}{R^{s}} \sigma R K_{g} \bar{I}_{b}\left[-F_{21} \cos \phi^{s}+F_{22} \sin \phi^{s}\right] \\
& a_{53}=-\sigma R K_{g} I_{g} \omega\left[F_{21} \sin \phi^{s}+F_{22} \cos \phi^{s}\right] \\
& a_{54}=-a_{34} \\
& b_{53}=1-b_{33} \\
& a_{66}=-a \\
& a_{55}=-a_{35} \\
& b_{54}=-b_{34} \\
& b_{66}=a \\
& a_{56}=-a_{36} \\
& b_{55}=-b_{35} \\
& c_{4}=-F_{11} \sigma\left(H \dot{V}+\omega V \tan \phi_{z}^{0}\right)-F_{12} \omega(\dot{V}+\sigma H V)+F_{11} \sigma R K_{g} \omega\left(\tilde{I}_{b} \cos \phi^{s}-I_{g} \sin \phi_{L}\right) \\
& +F_{12} \sigma R K_{g} \omega\left(-I_{b} \sin \phi^{s}+I_{g} \cos \phi_{L}\right)+\sigma R K_{g} \dot{I}_{g}\left(F_{11} \cos \phi_{L}+F_{12} \sin \phi_{L}\right) \\
& c_{5}=-F_{21} \sigma\left(H \dot{V}+\omega V \tan \phi_{z}^{0}\right)-F_{22} \omega(\dot{V}+\sigma H V)+F_{21} \sigma R K_{g} \omega\left(\tilde{I}_{b} \cos \phi^{s}-I_{g} \sin \phi_{L}\right) \\
& +F_{22} \sigma R K_{g} \omega\left(-\tilde{I}_{b} \sin \phi^{s}+I_{g} \cos \phi_{L}\right)+\sigma R K_{g} \dot{I}_{g}\left(F_{21} \cos \phi_{L}+F_{22} \sin \phi_{L}\right)
\end{aligned}
$$


TABLE I: A linear state-space control model. (Continued)

$$
\begin{aligned}
& c_{6}=-\left(a \phi_{z}^{0}+\dot{\phi}_{z}^{0}\right) \\
& F_{11}=\left(\sigma H V+\sigma R K_{g} \tilde{I}_{b} \sin \phi^{s}\right) / D \quad F_{21}=-\omega / D \\
& F_{12}=\left(\omega V+\sigma R K_{g} \tilde{I}_{b} \cos \phi^{s}\right) / D \quad F_{22}=\sigma H / D \\
& D=\omega\left(\omega V+\sigma R K_{g} \tilde{I}_{b} \cos \phi^{s}\right)+\sigma H\left(\sigma H V+\sigma R K_{g} \tilde{I}_{b} \sin \phi^{s}\right) \\
& A_{1}=\frac{\beta^{2} \gamma_{T}^{2} E}{R^{s}} \quad A_{3}=\frac{e V \beta c}{2 \pi R^{s}} \quad \tan \phi_{z}^{0}=\frac{\tilde{I}_{b}}{I_{0}} \cos \phi^{s} \quad I_{0}=\frac{V H}{R K_{g}} \\
& d_{11}=\frac{v^{s}}{\gamma^{2} B} \quad d_{21}=-\frac{\dot{A_{2}}}{A_{1}} \quad \tilde{I}_{b}=\frac{I_{b}}{K_{g}} \\
& d_{31}=\frac{2 \pi f}{\gamma^{2} B}\left[1+\tilde{I}_{b}\left\{F_{21} \cos \phi^{s}-F_{22} \sin \phi^{s}\right\}\right] \\
& d_{41}=\frac{2 \pi f_{1}}{\gamma^{2} B} \tilde{I}_{b}\left[-F_{11} \cos \phi^{s}+F_{12} \sin \phi^{s}\right] \\
& d_{51}=\frac{2 \pi f}{\gamma^{2} B} \tilde{I}_{b}\left[-F_{21} \cos \phi^{s}+F_{22} \sin \phi^{s}\right]
\end{aligned}
$$


TABLE II: Parameters used for normalization.

$$
\begin{gathered}
x_{1}^{m}=5 \mathrm{~m} \quad x_{2}^{m}=0.01 \mathrm{~m} \\
x_{3}^{m}=3^{\circ}=0.0524 \mathrm{rad} \quad x_{4}^{m}=8000 \mathrm{volts} \\
x_{5}^{m}=3^{\circ}=0.0524 \mathrm{rad} \quad x_{6}^{m}=3^{\circ}=0.0524 \mathrm{rad} \\
u_{1}^{m}=5000 \mathrm{~Hz}=u_{2}^{m}=u_{3}^{m} \\
x_{4}^{m}=5.7 \times 10^{-4} \mathrm{Amp} \\
x_{5}^{m}=0.1576 \mathrm{rad} \\
x_{6}^{m}=100 \mathrm{Amp} \\
K_{g}=700 \\
R=182 \mathrm{k} \Omega \\
Q=5000 \\
\phi_{L}=0 \\
\tilde{I}_{b}=100 \mathrm{~mA}
\end{gathered}
$$




\section{FIGURE CAPTIONS}

FIGURE 1. RF beam control loops planned for the SSC Low Energy Booster.

FIGURE 2. Equivalent circuit of the Cavity.

FIGURE 3. Schematic loop diagram of Figure 1 showing control and measured quantities.

FIGURE 4. Phasor diagram showing the relations between generator current, $i_{g}$, beam current, $i_{b}$, and the gap voltage, $v$ at a given time.

FIGURE 5. Cavity tuning loop (without feedforward control).

FIGURE 6. Variation of mean radial orbit with time for $10 \mathrm{MeV}$ injection energy error predicted using tracking code and the non-linear model.

FIGURE 7. Variation of mean radial orbit shift with time (expanded scale of Figure 6).

FIGURE 8. Variation of particle phase with time for $10 \mathrm{MeV}$ injection energy error predicted using tracking code and the non-linear model.

FIGURE 9. Variation of particle phase with time (expanded scale of Figure 8).

FIGURE 10. Variation of voltage error (gap voltage-reference voltage) with time predicted using tracking code and the non-linear model.

FIGURE 11. Variation of cavity gap voltage error with time (expanded scale of Figure 10).

FIGURE 12. Variation of cavity gap phase error with time (expanded scale).

FIGURE 13. Gain plots of $\mathrm{G}_{11}(\mathrm{~s}), \mathrm{G}_{14}(\mathrm{~s})$ and $\mathrm{G}_{15}(\mathrm{~s})$ with frequency (open loop gain of synchronization loop due to controls $\Delta F^{c}(s), \Delta I^{c}(s)$, and $\Delta \phi^{c}(s)$ at $1 \mathrm{~ms}, 10 \mathrm{~ms}, 20 \mathrm{~ms}$ and $45 \mathrm{~ms}$ for $H=1$ and $I_{b}=100 \mathrm{~mA}$ ). 
FIGURE 14. Gain plots of $\mathrm{G}_{22}(\mathrm{~s}), \mathrm{G}_{24}(\mathrm{~s})$ and $\mathrm{G}_{25}$ (s) with frequency (open loop gain of radial loop due to controls $\Delta F^{c}(s), \Delta I^{c}(s)$, and $\Delta \phi^{c}(s)$ at $1 \mathrm{~ms}, 10 \mathrm{~ms}, 20 \mathrm{~ms}$ and $45 \mathrm{~ms}$ for $H=1$ and $\left.I_{b}=100 \mathrm{~mA}\right)$.

FIGURE 15. Gain plots of $\mathrm{G}_{33}(\mathrm{~s}), \mathrm{G}_{34}(\mathrm{~s})$ and $\mathrm{G}_{35}(\mathrm{~s})$ with frequency (open loop gain of beam phase loop due to controls $\Delta F^{c}(s), \Delta l^{c}(s)$, and $\Delta \phi^{c}(s)$ at $1 \mathrm{~ms}, 10 \mathrm{~ms}, 20 \mathrm{~ms}$ and $45 \mathrm{~ms}$ for $H=1$ and $I_{b}=100 \mathrm{~mA}$ ).

FIGURE 16. Gain plots of $\mathrm{G}_{41}(\mathrm{~s}), \mathrm{G}_{44}(\mathrm{~s})$ and $\mathrm{G}_{45}(\mathrm{~s})$ with frequency (open loop gain of local amplitude loop due to controls $\Delta F^{c}(s), \Delta I^{c}(s)$, and $\Delta \phi^{c}(s)$ at $1 \mathrm{~ms}, 10 \mathrm{~ms}, 20 \mathrm{~ms}$ and $45 \mathrm{~ms}$ for $H=1$ and $I_{b}=100 \mathrm{~mA}$ ).

FIGURE 17. Gain plots of $\mathrm{G}_{51}(\mathrm{~s}), \mathrm{G}_{54}(\mathrm{~s})$ and $\mathrm{G}_{55}(\mathrm{~s})$ with frequency (open loop gain of local phase loop due to controls $\Delta F^{c}(s), \Delta I^{c}(s)$, and $\Delta \phi^{c}(s)$ at $1 \mathrm{~ms}, 10 \mathrm{~ms}, 20 \mathrm{~ms}$ and $45 \mathrm{~ms}$ for $H=1$ and $I_{b}=100 \mathrm{~mA}$ ).

FIGURE 18. Gain plots of $\mathrm{G}_{16}(\mathrm{~s}), \mathrm{G}_{26}(\mathrm{~s}), \mathrm{G}_{36}(\mathrm{~s}), \mathrm{G}_{46}(\mathrm{~s}), \mathrm{G}_{56}(\mathrm{~s})$ and $\mathrm{G}_{66}(\mathrm{~s})$ with frequency (open loop gain of all six loops with respect to tuning control, $I_{\text {tune }}^{c}(s)$ at $1 \mathrm{~ms}, 10 \mathrm{~ms}, 20 \mathrm{~ms}$ and $45 \mathrm{~ms}$ for $H=1$ and $I_{b}=100 \mathrm{~mA}$ ).

FIGURE 19. Gain plots of $\mathrm{G}_{11}(\mathrm{~s}), \mathrm{G}_{14}(\mathrm{~s})$ and $\mathrm{G}_{15}(\mathrm{~s})$ with frequency (open loop gain of synchronization loop due to controls $\Delta F^{c}(s), \Delta I^{c}(s)$, and $\Delta \phi^{c}(s)$ at $10 \mathrm{~ms}$, for $H=1,10,20,30$ and $I_{b}=100 \mathrm{~mA}$ ).

FIGURE 20. Gain plots of $\mathrm{G}_{22}(\mathrm{~s}), \mathrm{G}_{24}(\mathrm{~s})$ and $\mathrm{G}_{25}$ (s) with frequency (open loop gain of radial loop due to controls $\Delta F^{c}(s), \Delta I^{c}(s)$, and $\Delta \phi^{c}(s)$ at $10 \mathrm{~ms}$, for $H=1,10,20,30$ and $\left.I_{b}=100 \mathrm{~mA}\right)$.

FIGURE 21. Gain plots of $\mathrm{G}_{33}(\mathrm{~s}), \mathrm{G}_{34}(\mathrm{~s})$ and $\mathrm{G}_{35}(\mathrm{~s})$ with frequency (open loop gain of beam phase loop due to controls $\Delta F^{c}(s), \Delta I^{c}(s)$, and $\Delta \phi^{c}(s)$ at $10 \mathrm{~ms}$, for $H=1,10,20,30$ and $\left.I_{b}=100 \mathrm{~mA}\right)$. 
FIGURE 22. Gain plots of $\mathrm{G}_{41}(\mathrm{~s}), \mathrm{G}_{44}(\mathrm{~s})$ and $\mathrm{G}_{45}(\mathrm{~s})$ with frequency (open loop gain of local amplitude loop due to controls $\Delta F^{c}(s), \Delta I^{c}(s)$, and $\Delta \phi^{c}(s)$ at $10 \mathrm{~ms}$, for $H=1,10,20,30$ and $\left.I_{b}=100 \mathrm{~mA}\right)$.

FIGURE 23. Gain plots of $\mathrm{G}_{51}(\mathrm{~s}), \mathrm{G}_{54}(\mathrm{~s})$ and $\mathrm{G}_{55}(\mathrm{~s})$ with frequency (open loop gain of local phase loop due to controls $\Delta F^{c}(s), \Delta I^{c}(s)$, and $\Delta \phi^{c}(s)$ at $10 \mathrm{~ms}$, for $H=1,10,20,30$ and $\left.I_{b}=100 \mathrm{~mA}\right)$.

FIGURE 24. Gain plots of $\mathrm{G}_{16}(\mathrm{~s}), \mathrm{G}_{26}(\mathrm{~s}), \mathrm{G}_{36}(\mathrm{~s}), \mathrm{G}_{46}(\mathrm{~s}), \mathrm{G}_{56}(\mathrm{~s})$ and $\mathrm{G}_{66}(\mathrm{~s})$ with frequency (open loop gain of all six loops with respect to tuning control, $I_{\text {tune }}^{c}(s)$ at $10 \mathrm{~ms}$ for $\mathrm{H}=1,10,20$, 30 and $\left.I_{b}=100 \mathrm{~mA}\right)$.

FIGURE 25. Gain plots of $\mathrm{G}_{11}(\mathrm{~s}), \mathrm{G}_{14}(\mathrm{~s})$ and $\mathrm{G}_{15}(\mathrm{~s})$ with frequency (open loop gain of synchronization loop due to controls $\Delta F^{c}(s), \Delta I^{c}(s)$, and $\Delta \phi^{c}(s)$ at $10 \mathrm{~ms}$, for $H=20$ and $\left.I_{b}=1,2,3 \mathrm{~A}\right)$.

FIGURE 26. Gain plots of $\mathrm{G}_{22}(\mathrm{~s}), \mathrm{G}_{24}(\mathrm{~s})$ and $\mathrm{G}_{25}(\mathrm{~s})$ with frequency (open loop gain of radial loop due to controls $\Delta F^{c}(s), \Delta I^{c}(s)$, and $\Delta \phi^{c}(s)$ at $10 \mathrm{~ms}$, for $H=20$ and $I_{b}=1,2,3 \mathrm{~A}$ ).

FIGURE 27. Gain plots of $\mathrm{G}_{33}(\mathrm{~s}), \mathrm{G}_{34}(\mathrm{~s})$ and $\mathrm{G}_{35}(\mathrm{~s})$ with frequency (open loop gain of beam phase loop due to controls $\Delta F^{c}(s), \Delta I^{c}(s)$, and $\Delta \phi^{c}(s)$ at $10 \mathrm{~ms}$, for $H=20$ and $\left.l_{b}=1,2,3 \mathrm{~A}\right)$.

FIGURE 28. Gain plots of $\mathrm{G}_{41}(\mathrm{~s}), \mathrm{G}_{44}(\mathrm{~s})$ and $\mathrm{G}_{45}(\mathrm{~s})$ with frequency (open loop gain of local amplitude loop due to controls $\Delta F^{c}(s), \Delta I^{c}(s)$, and $\Delta \phi^{c}(s)$ at $10 \mathrm{~ms}$, for $H=20$ and $\left.I_{b}=0,1,2,3 \mathrm{~A}\right)$.

FIGURE 29. Gain plots of $\mathrm{G}_{51}(\mathrm{~s}), \mathrm{G}_{54}(\mathrm{~s})$ and $\mathrm{G}_{55}(\mathrm{~s})$ with frequency (open loop gain of local phase loop due to controls $\Delta F^{c}(s), \Delta I^{c}(s)$, and $\Delta \phi^{c}(s)$ at $10 \mathrm{~ms}$, for $H=20$ and $\left.I_{b}=0,1,2,3 \mathrm{~A}\right)$. 
FIGURE 30. Gain plots of $\mathrm{G}_{16}(\mathrm{~s}), \mathrm{G}_{26}(\mathrm{~s}), \mathrm{G}_{36}(\mathrm{~s}), \mathrm{G}_{46}(\mathrm{~s}), \mathrm{G}_{56}(\mathrm{~s})$ and $\mathrm{G}_{66}(\mathrm{~s})$ with frequency (open loop gain of all six loops with respect to tuning control, $I_{\text {tune }}^{c}(s)$ at $10 \mathrm{~ms}$ for $H=20$ and $\left.I_{b}=0,1,2,3 \mathrm{~A}\right)$.

FIGURE A.1. Cavity gap voltage tracked using the representations $i_{g}=I_{g} e^{j \omega t}$ and $i_{g}=I_{g} e^{j \int \omega d t}$ for the complete acceleration cycle of the LEB. 


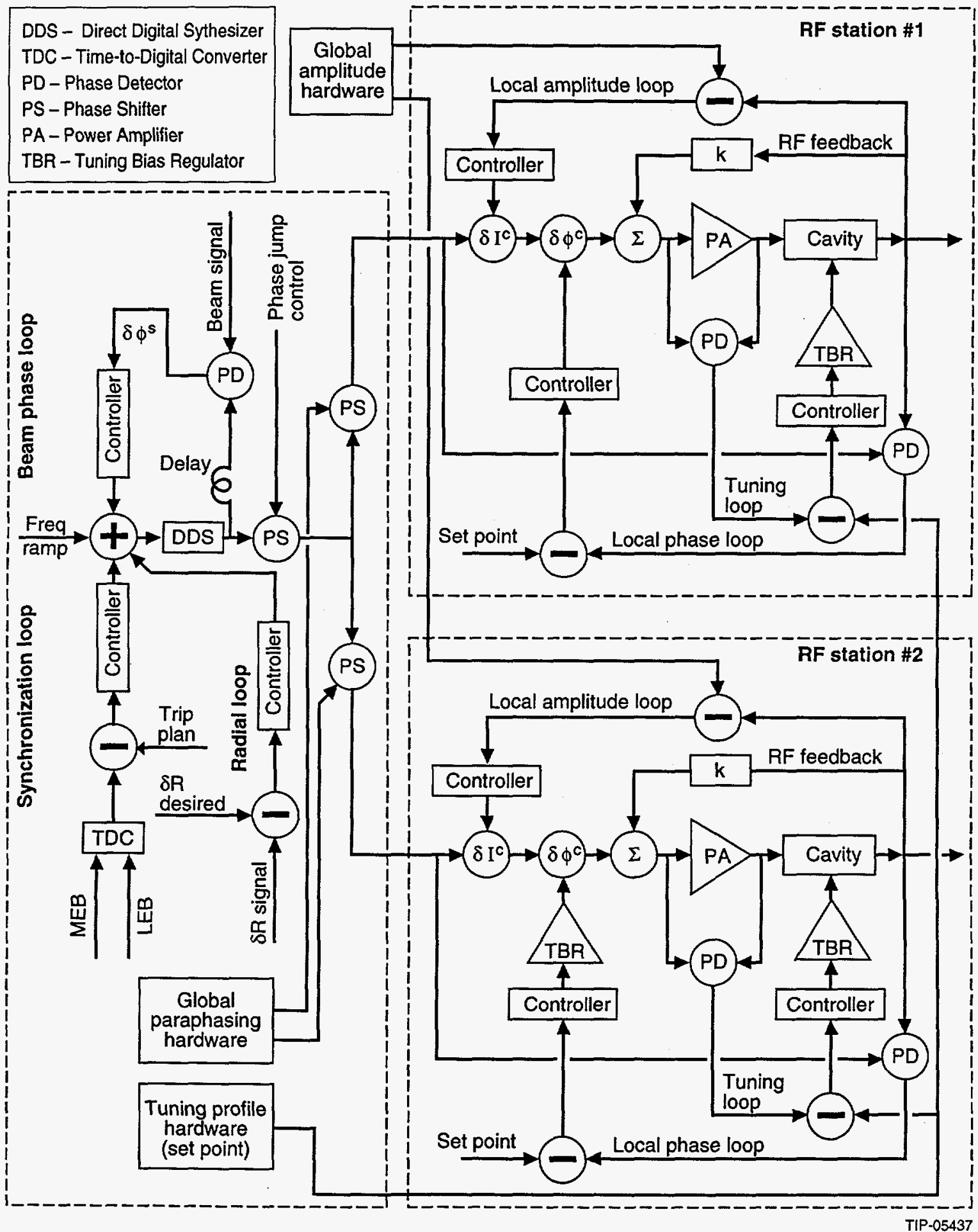

FIGURE 1. RF beam control loops planned for the SSC Low Energy Booster. 


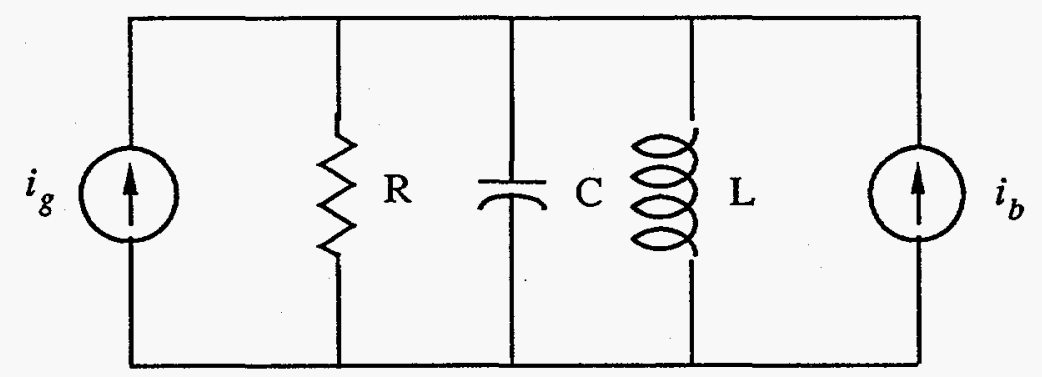

FIGURE 2. Equivalent circuit of the Cavity. 


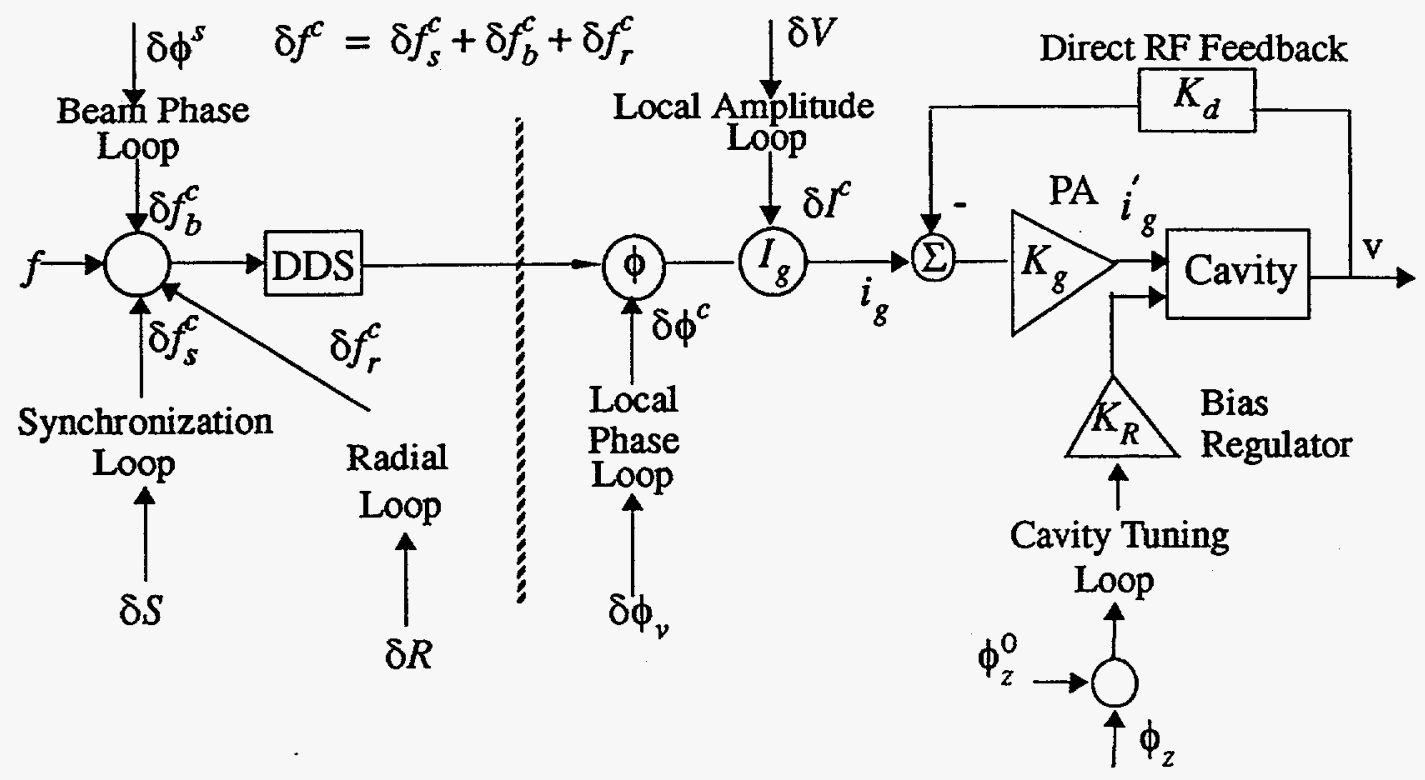

FIGURE 3. Schematic loop diagram of Figure 1 showing control and measured quantities.

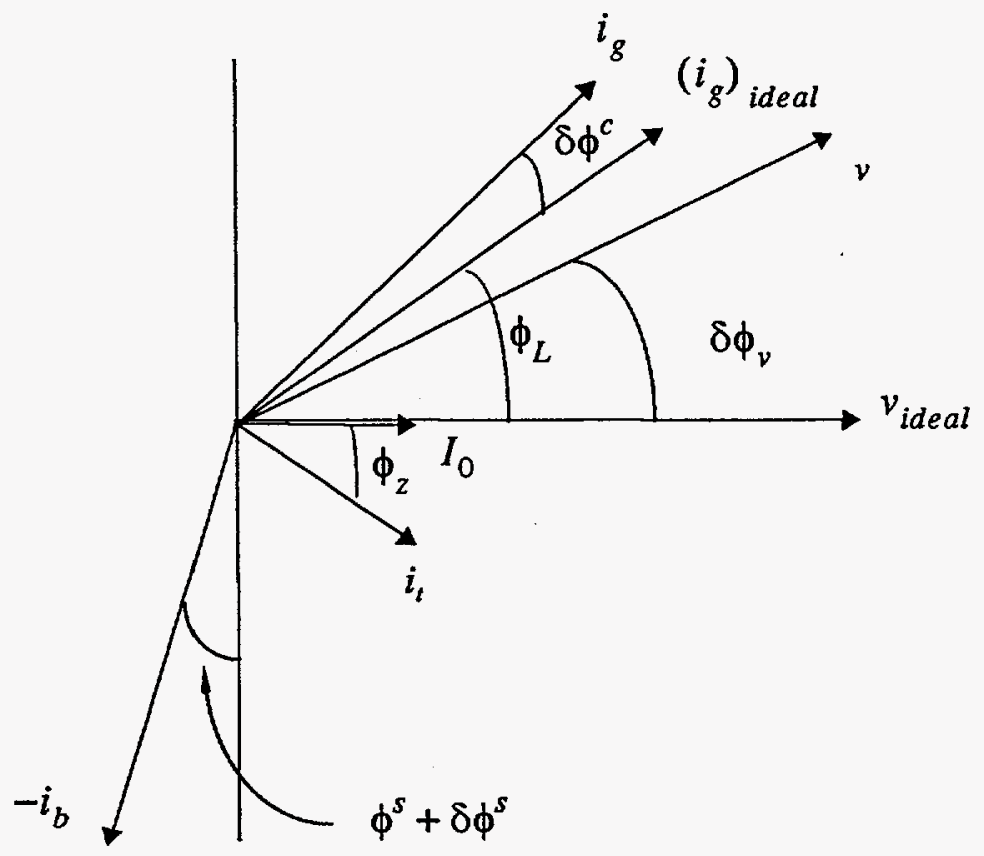

FIGURE 4. Phasor diagram showing the relations between generator current, $i_{g}$, beam current, $i_{b}$, and the gap voltage, $v$ at a given time. 


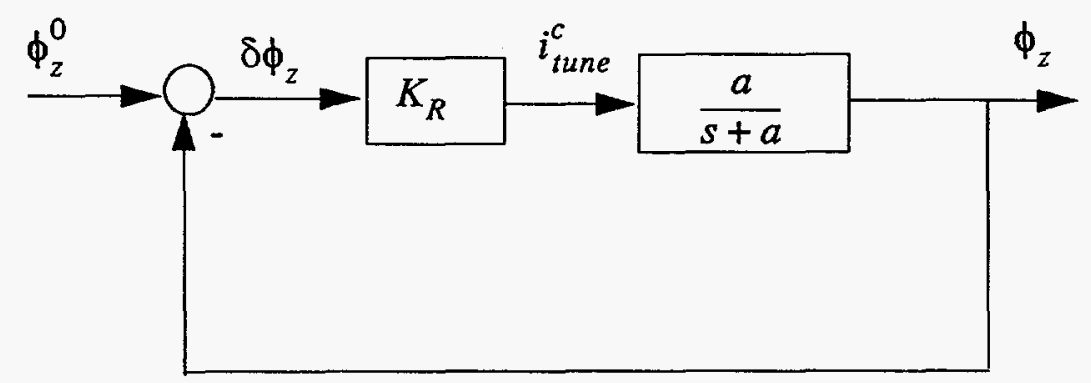

FIGURE 5. Cavity tuning loop (without feedforward control). 

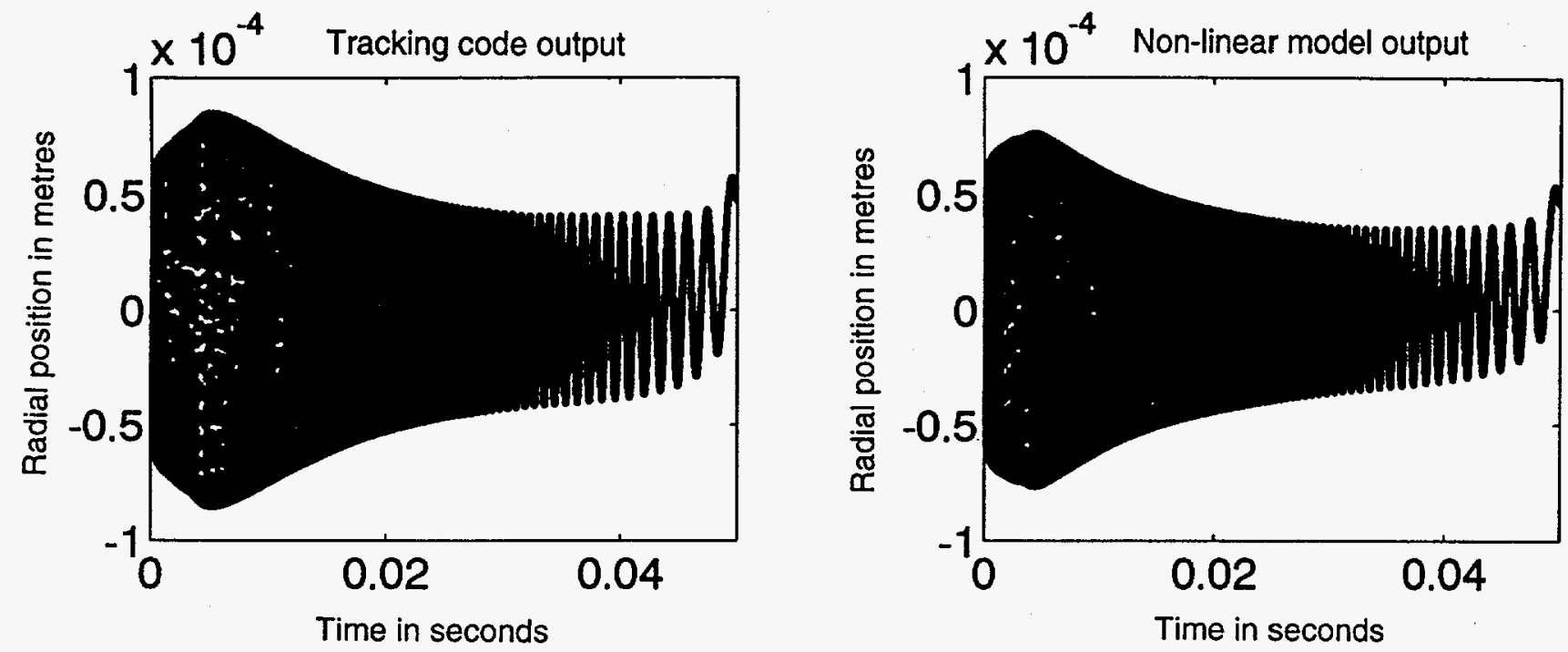

FIGURE 6. Variation of mean radial orbit with time for $10 \mathrm{MeV}$ injection energy error predicted using tracking code and the non-linear model. 

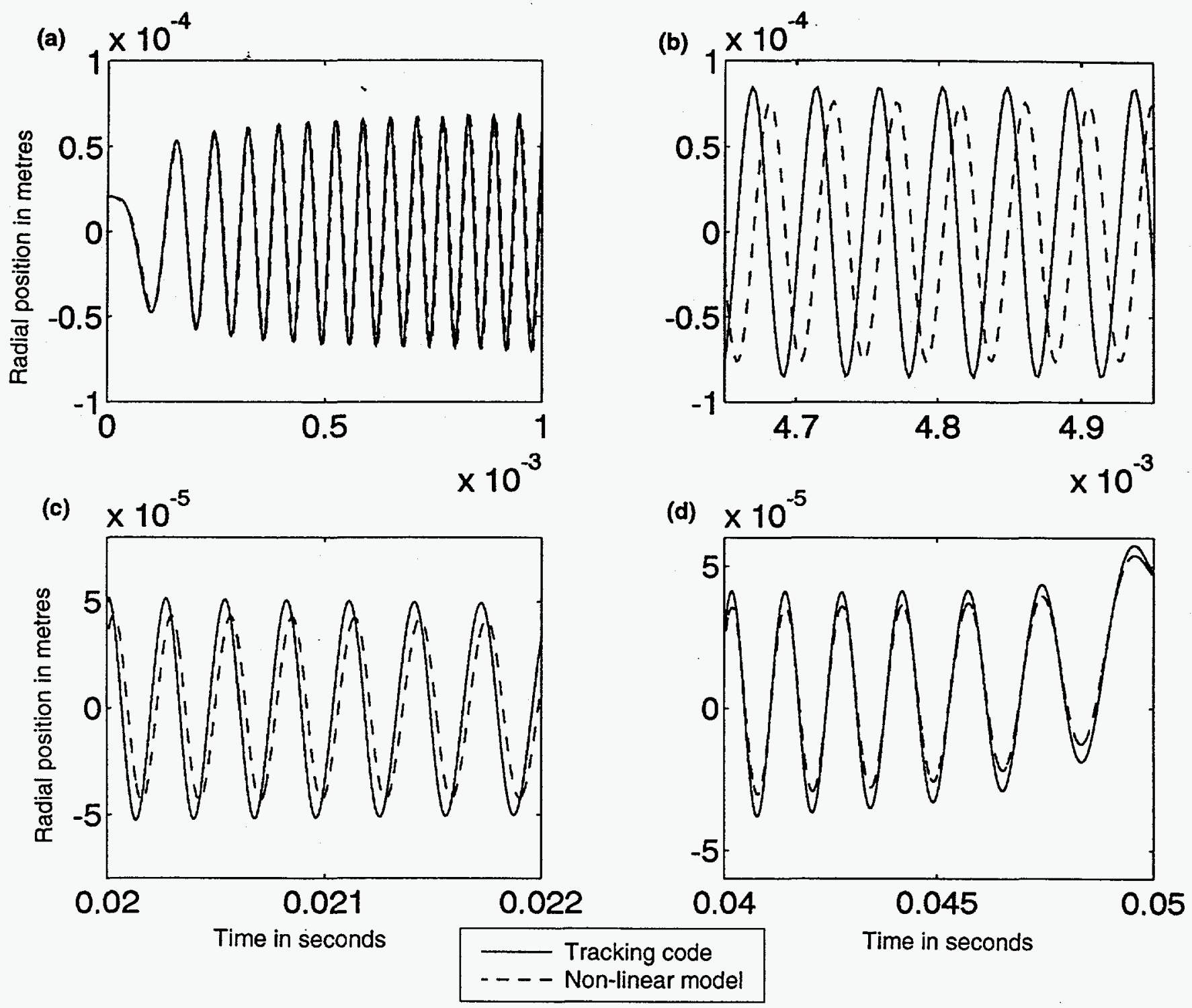

FIGURE 7. Variation of mean radial orbit shift with time (expanded scale of Figure 6). 

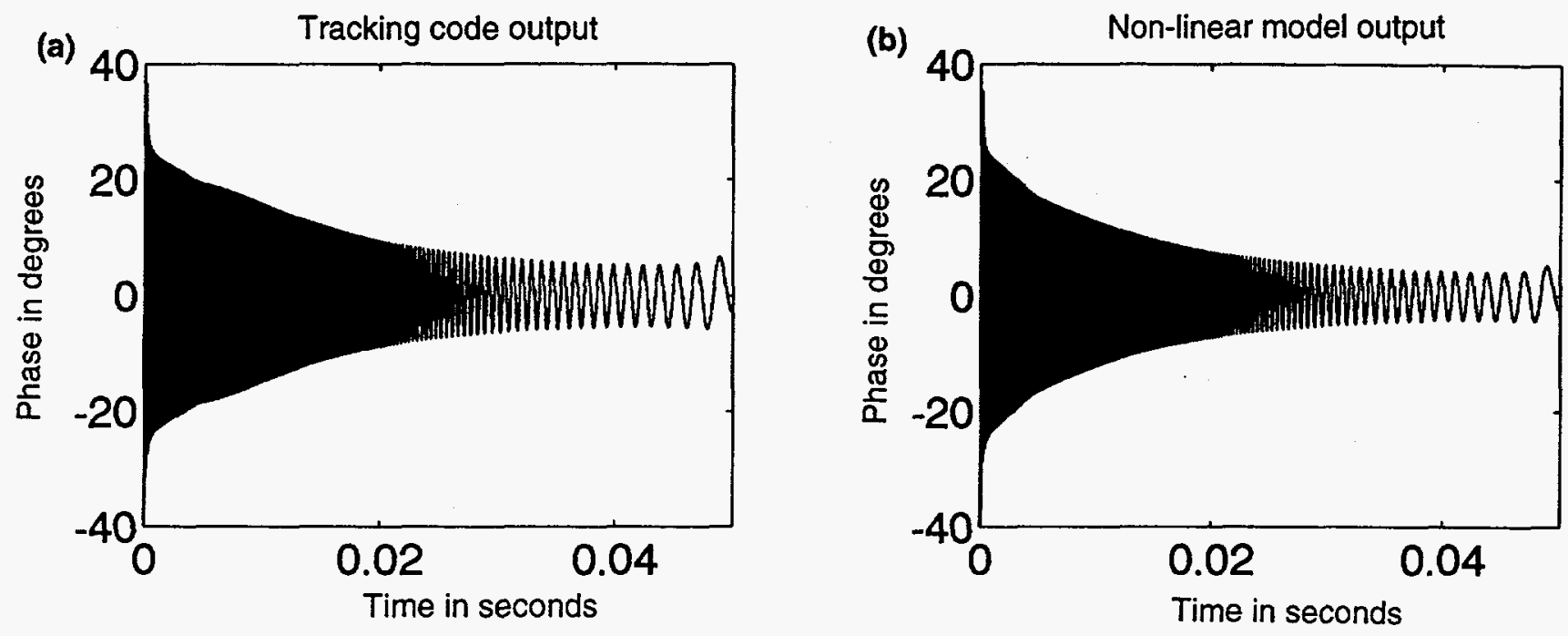

FIGURE 8. Variation of particle phase with time for $10 \mathrm{MeV}$ injection energy error predicted using tracking code and the non-linear model. 

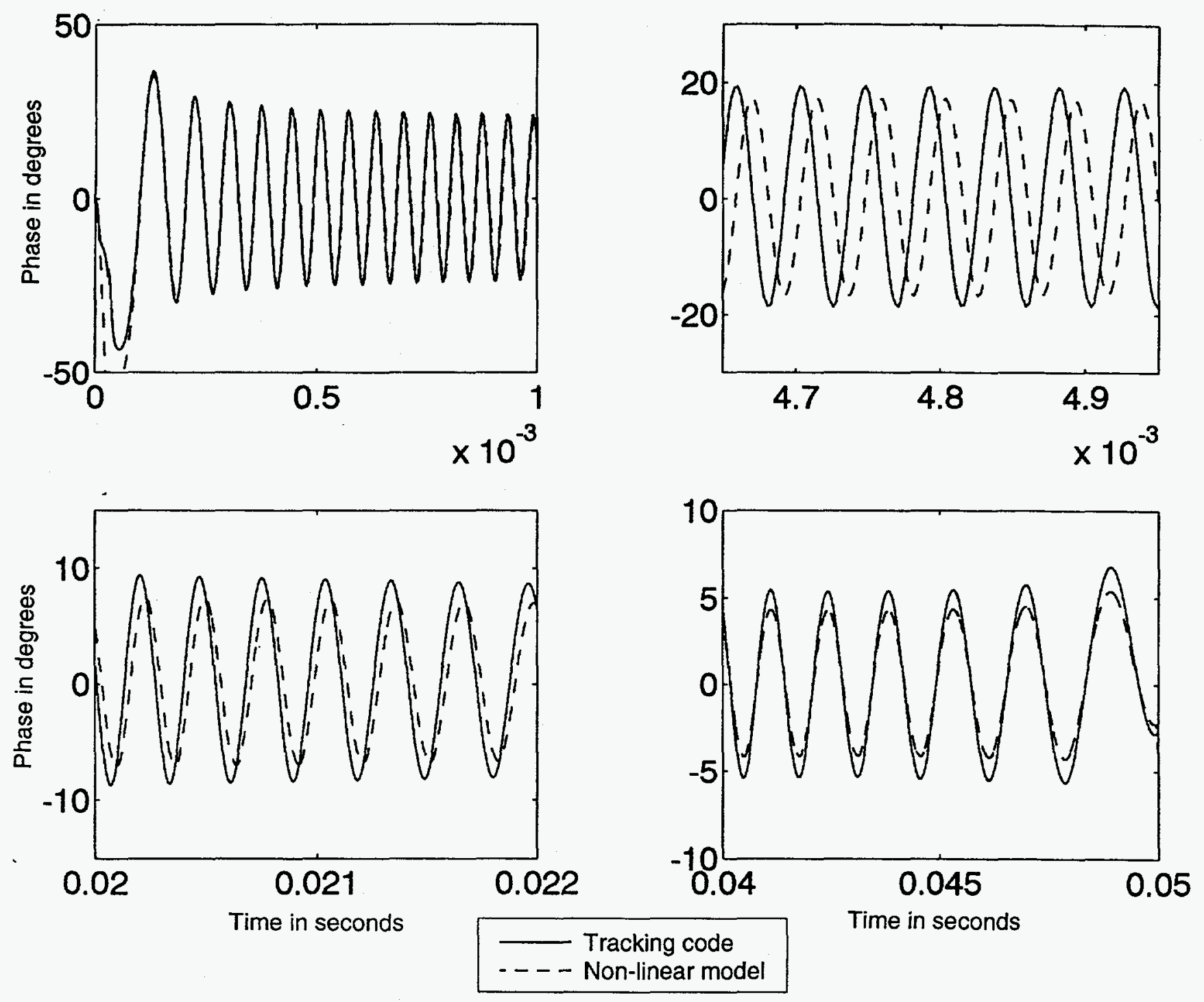

FIGURE 9. Variation of particle phase with time (expanded scale of Figure 8). 

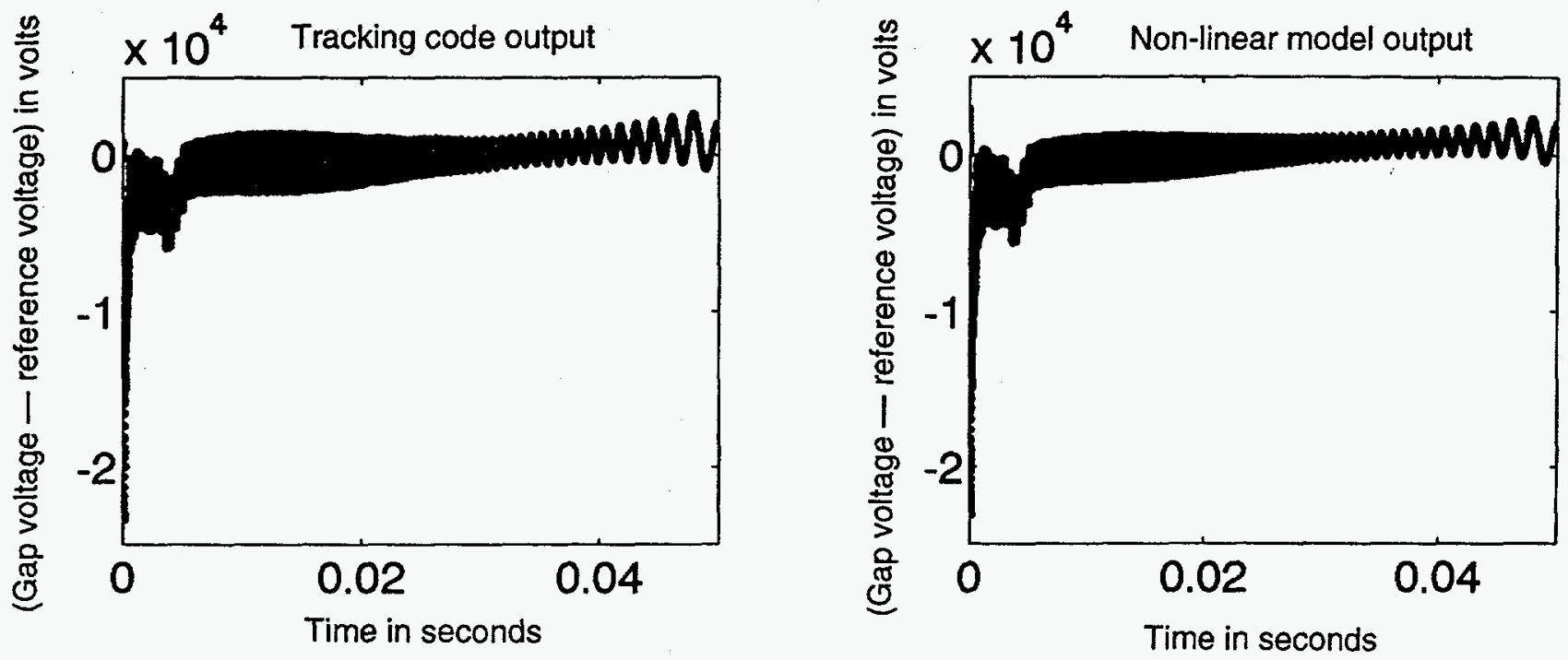

FIGURE 10. Variation of voltage error (gap voltage-reference voltage) with time predicted using tracking code and the non-linear model. 

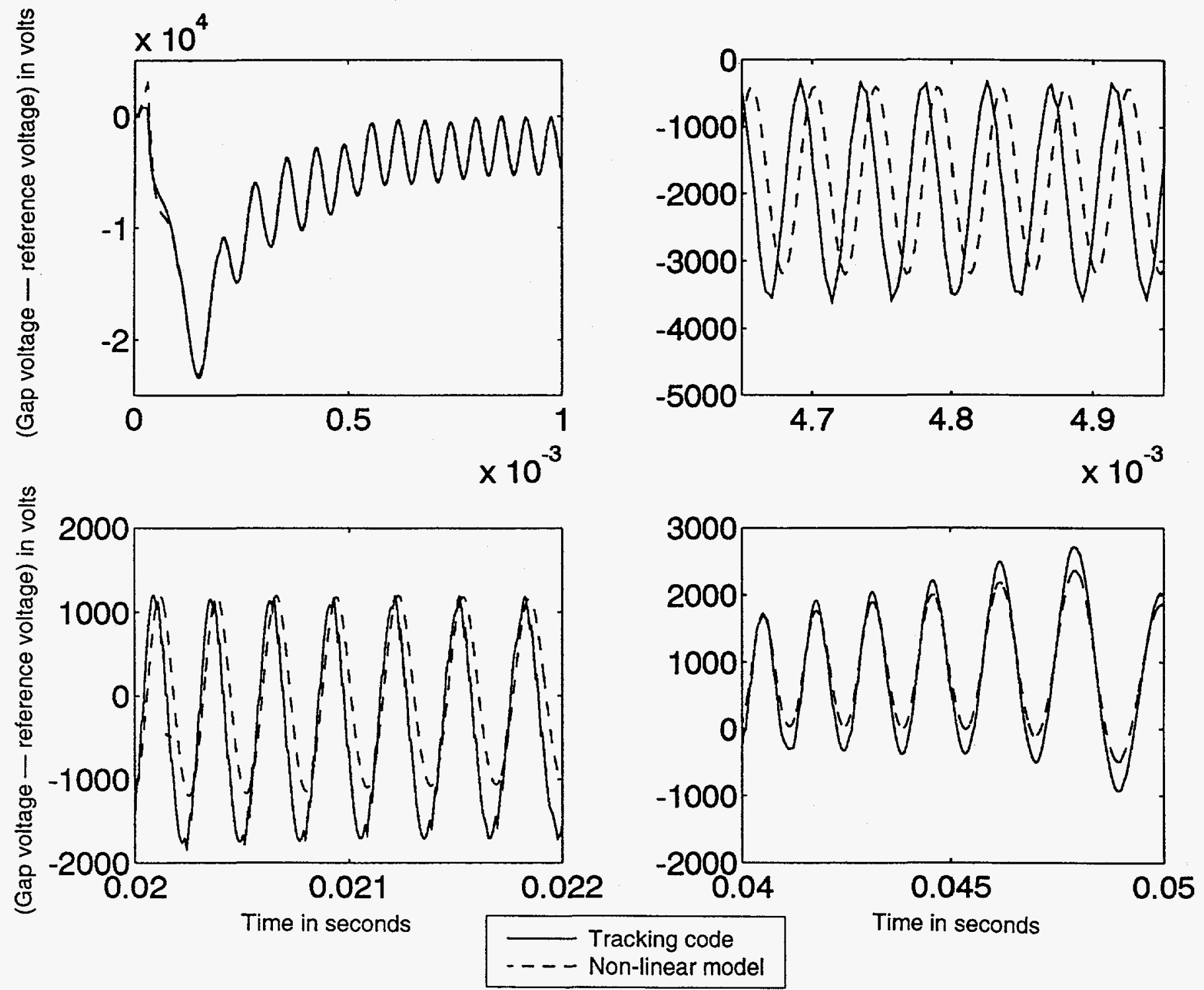

FIGURE 11. Variation of cavity gap voltage error with time (expanded scale of Figure 10). 

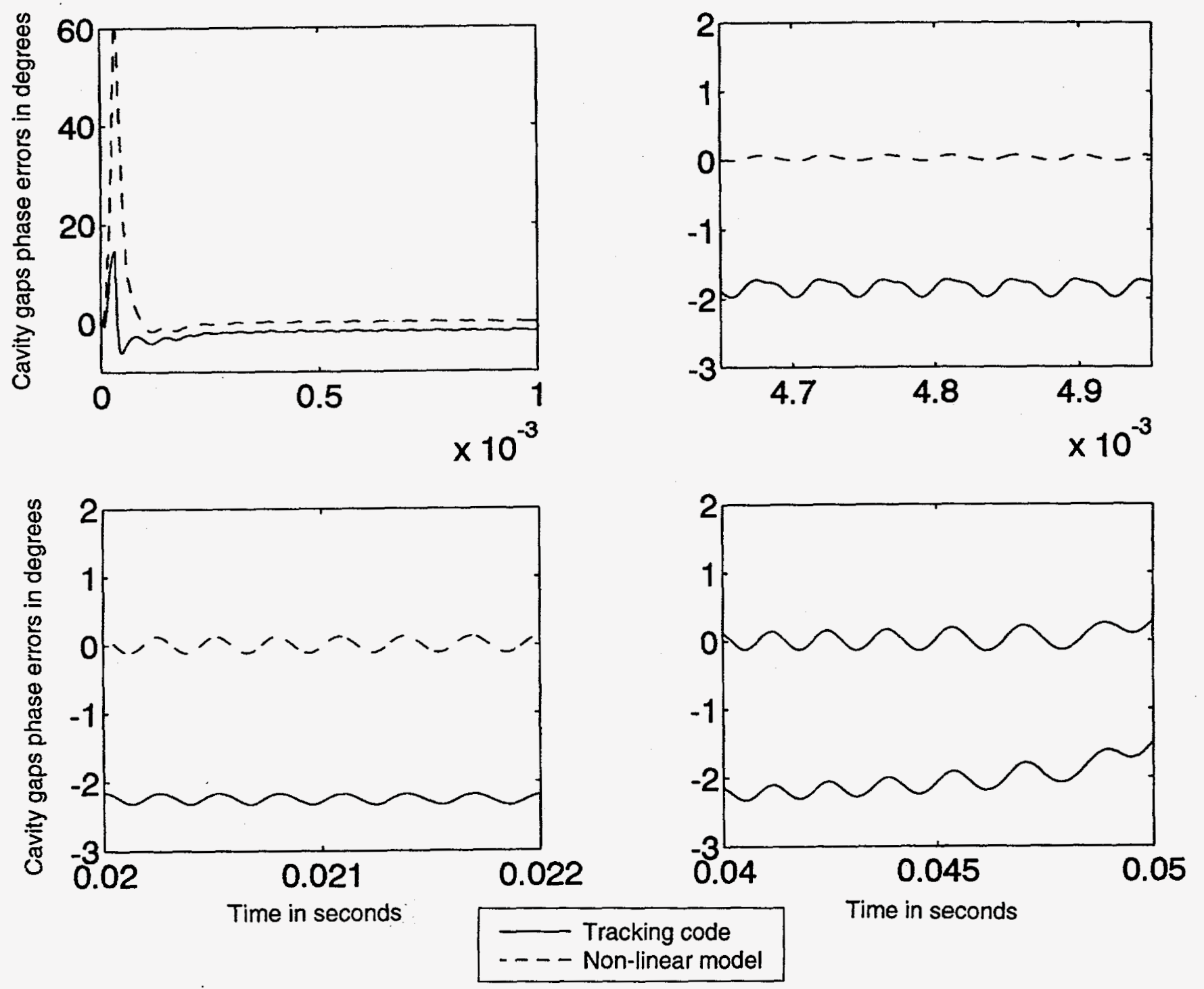

FIGURE 12. Variation of cavity gap phase error with time (expanded scale). 

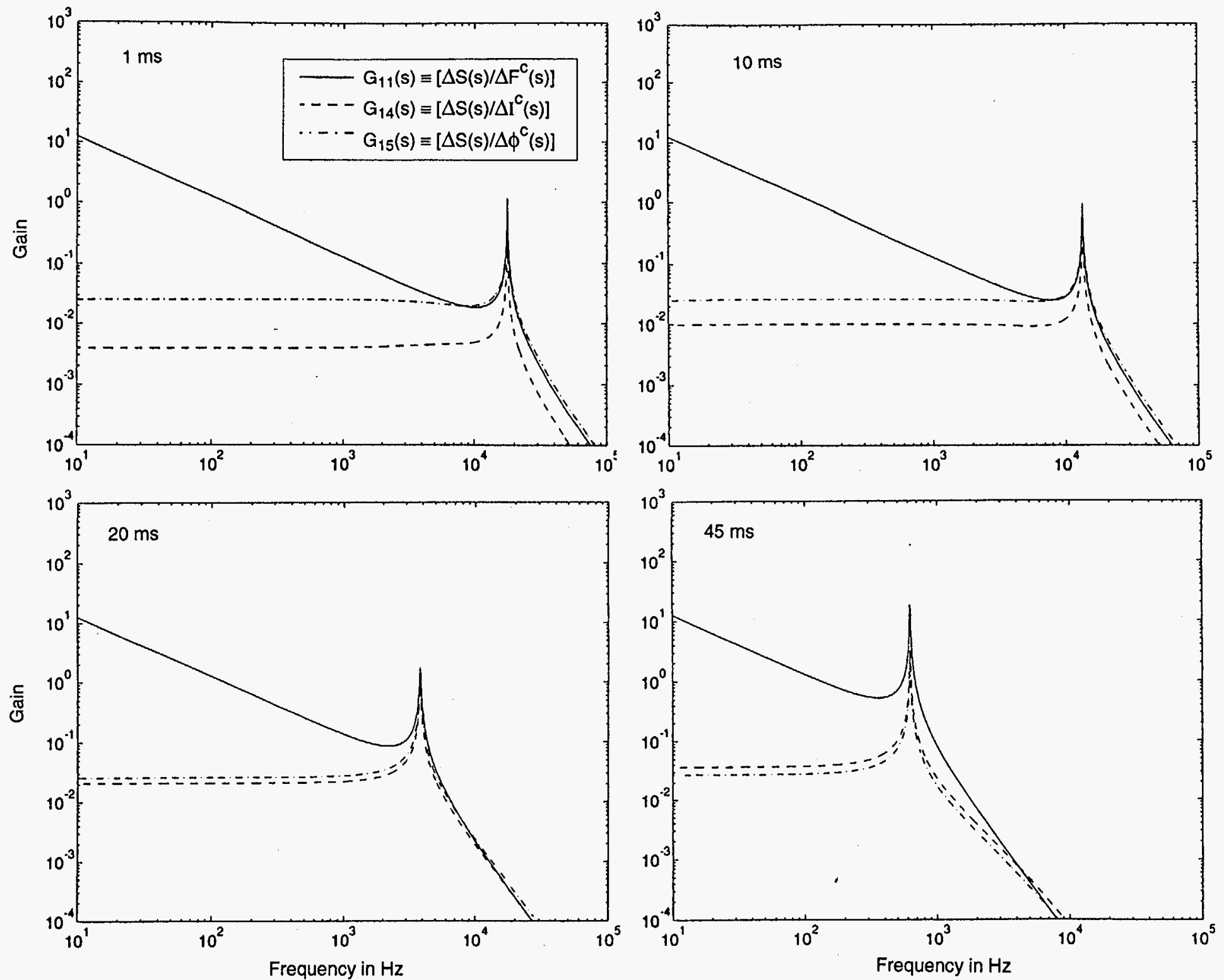

FIGURE 13. Gain plots of $\mathrm{G}_{11}(\mathrm{~s}), \mathrm{G}_{14}(\mathrm{~s})$ and $\mathrm{G}_{15}(\mathrm{~s})$ with frequency (open loop gain of synchronization loop due to controls $\Delta F^{c}(s), \Delta I^{c}(s)$, and $\Delta \phi^{c}(s)$ at $1 \mathrm{~ms}, 10 \mathrm{~ms}, 20 \mathrm{~ms}$ and $45 \mathrm{~ms}$ for $H=1$ and $I_{b}=100 \mathrm{~mA}$ ). 

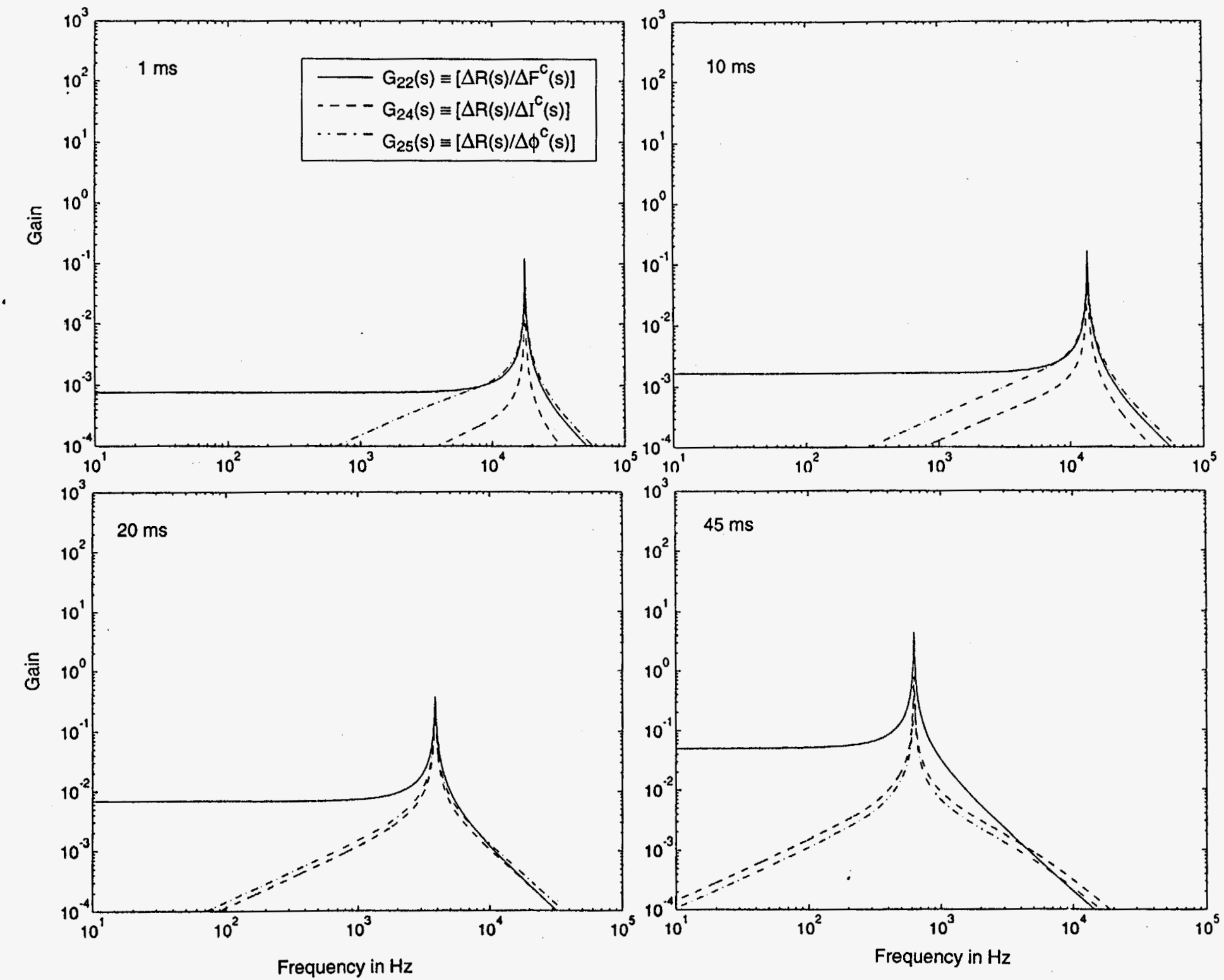

FIGURE 14. Gain plots of $\mathrm{G}_{22}(\mathrm{~s}), \mathrm{G}_{24}(\mathrm{~s})$ and $\mathrm{G}_{25}(\mathrm{~s})$ with frequency (open loop gain of radial loop due to controls $\Delta F^{c}(s), \Delta I^{c}(s)$, and $\Delta \phi^{c}(s)$ at $1 \mathrm{~ms}, 10 \mathrm{~ms}, 20 \mathrm{~ms}$ and $45 \mathrm{~ms}$ for $H=1$ and $\left.I_{b}=100 \mathrm{~mA}\right)$. 

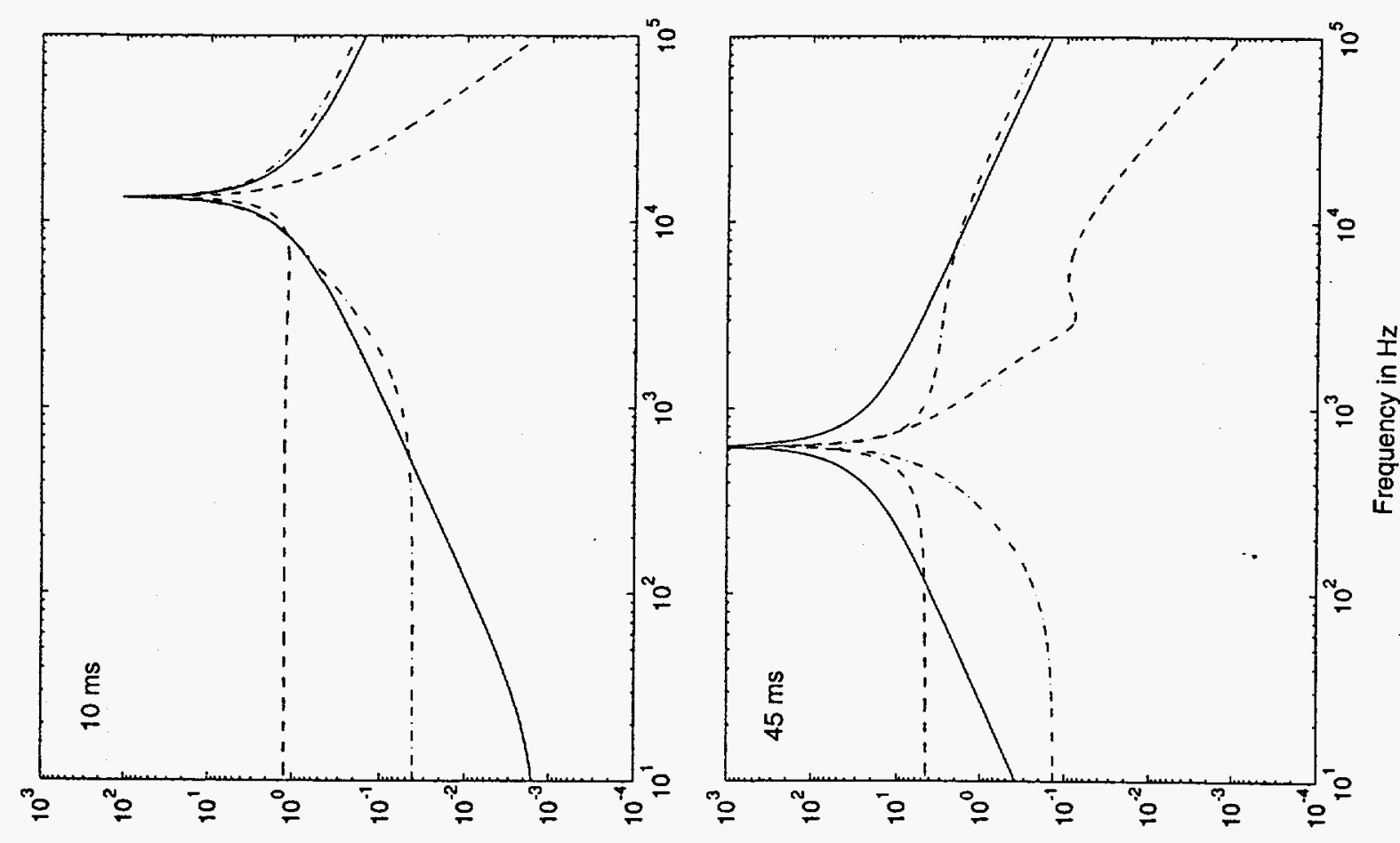

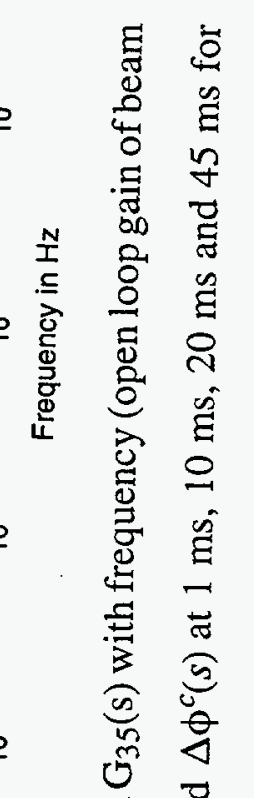
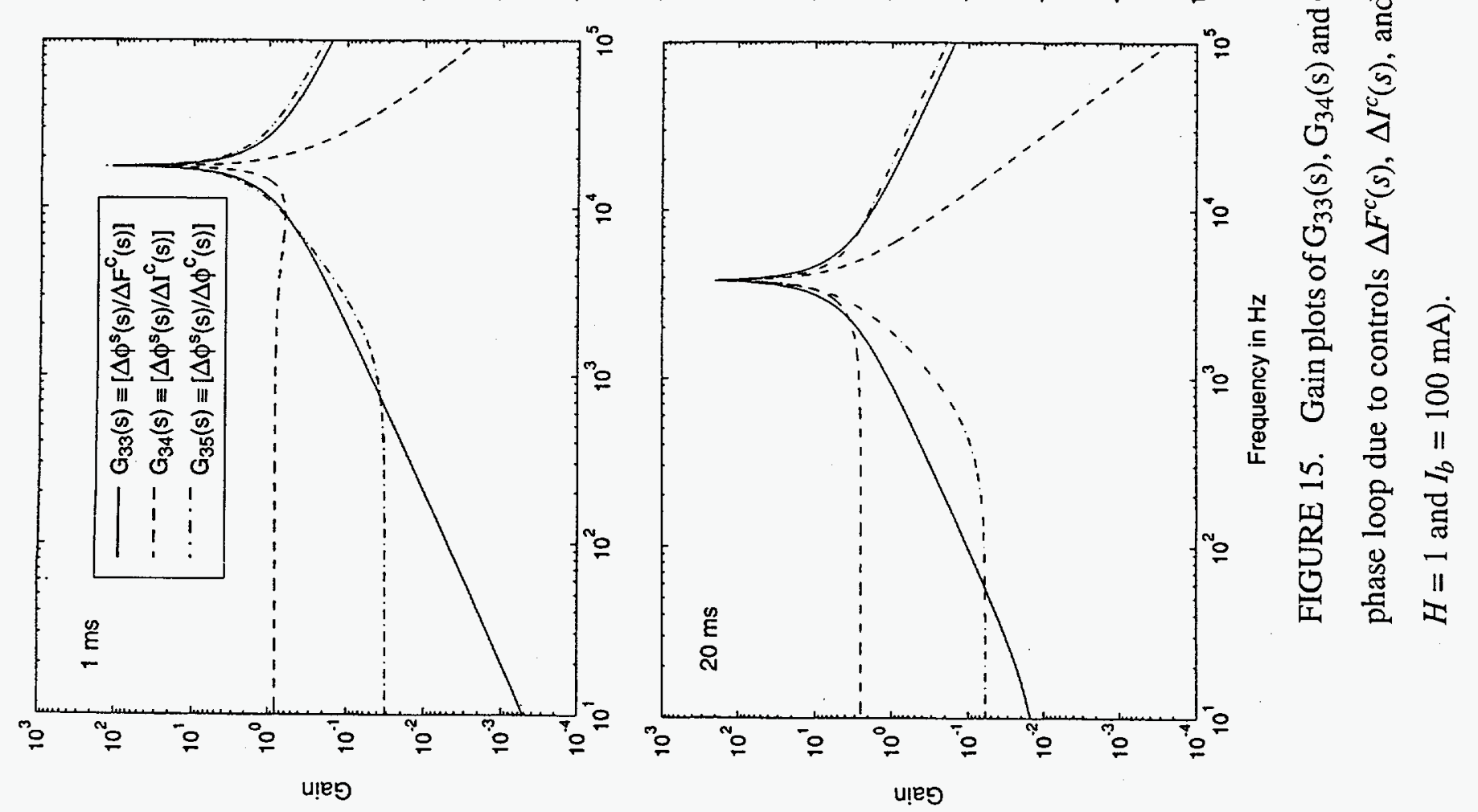

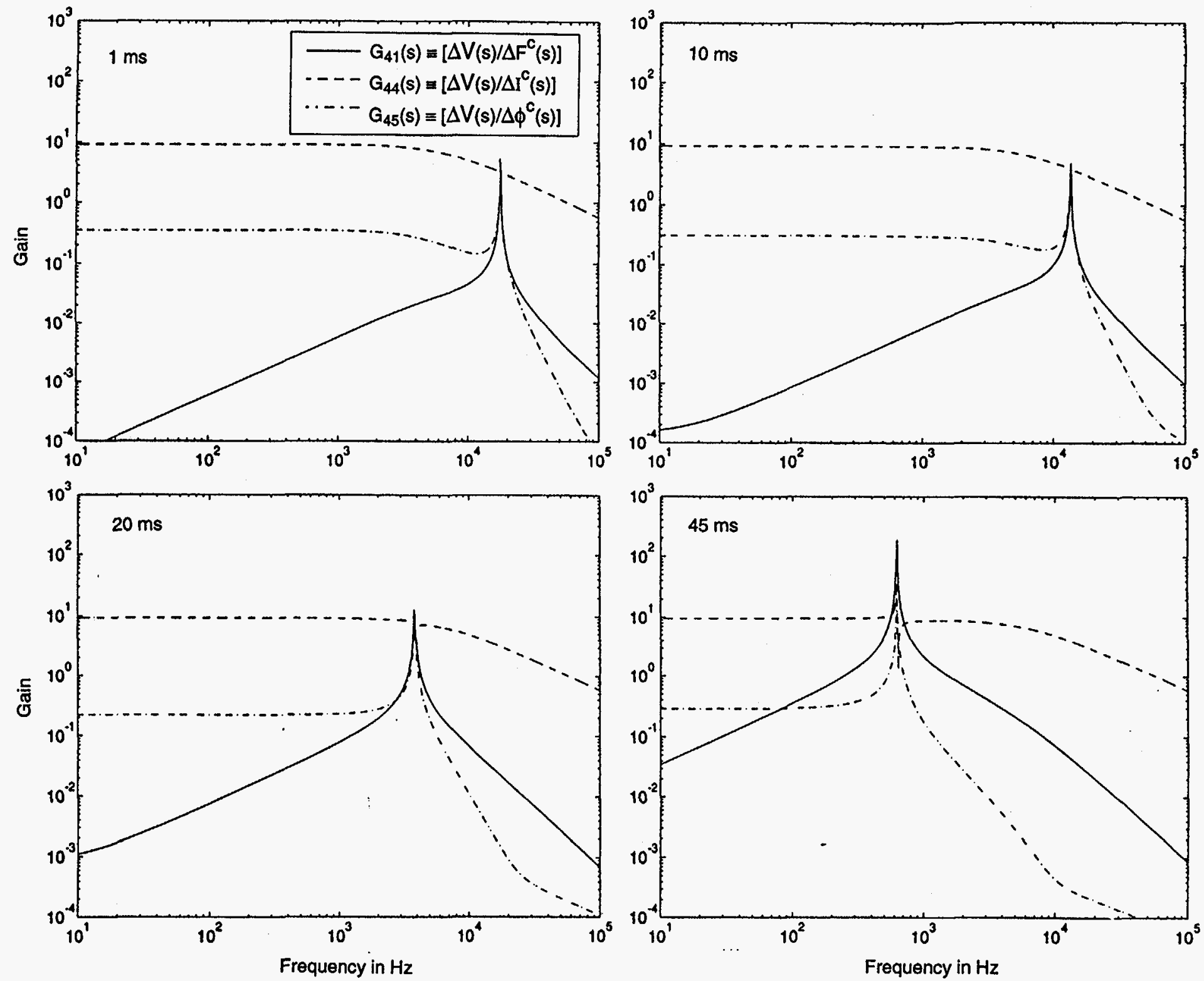

FIGURE 16. Gain plots of $\mathrm{G}_{41}(\mathrm{~s}), \mathrm{G}_{44}(\mathrm{~s})$ and $\mathrm{G}_{45}(\mathrm{~s})$ with frequency (open loop gain of local amplitude loop due to controls $\Delta F^{c}(s), \Delta I^{c}(s)$, and $\Delta \phi^{c}(s)$ at $1 \mathrm{~ms}, 10 \mathrm{~ms}, 20 \mathrm{~ms}$ and $45 \mathrm{~ms}$ for $H=1$ and $\left.I_{b}=100 \mathrm{~mA}\right)$. 

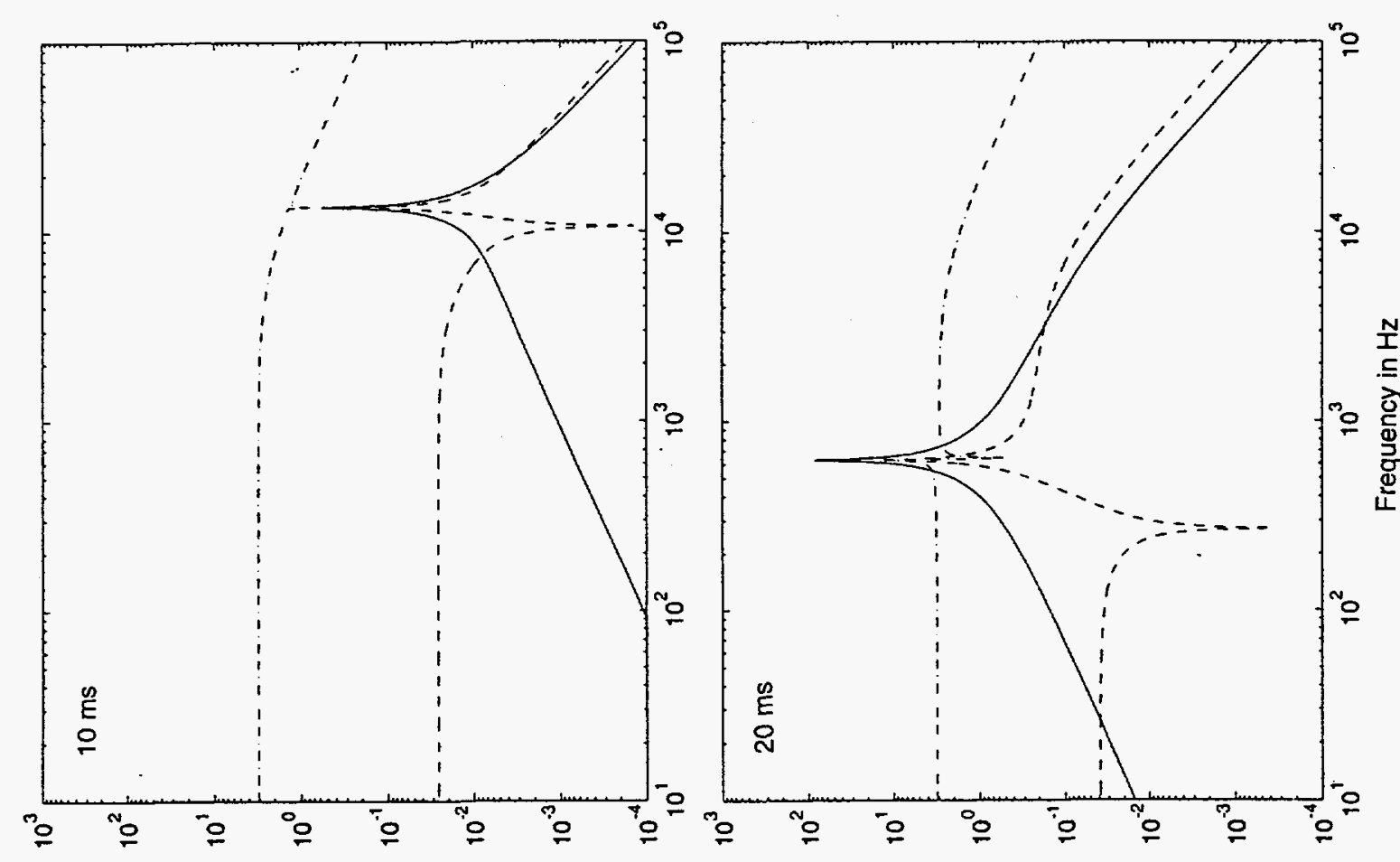

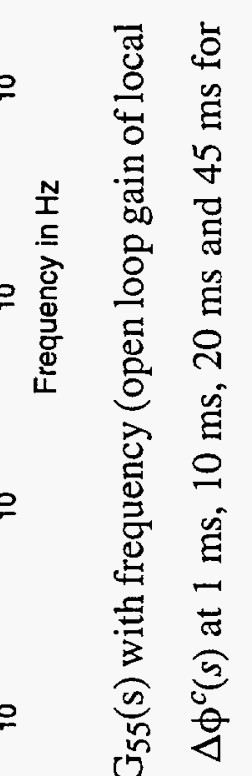

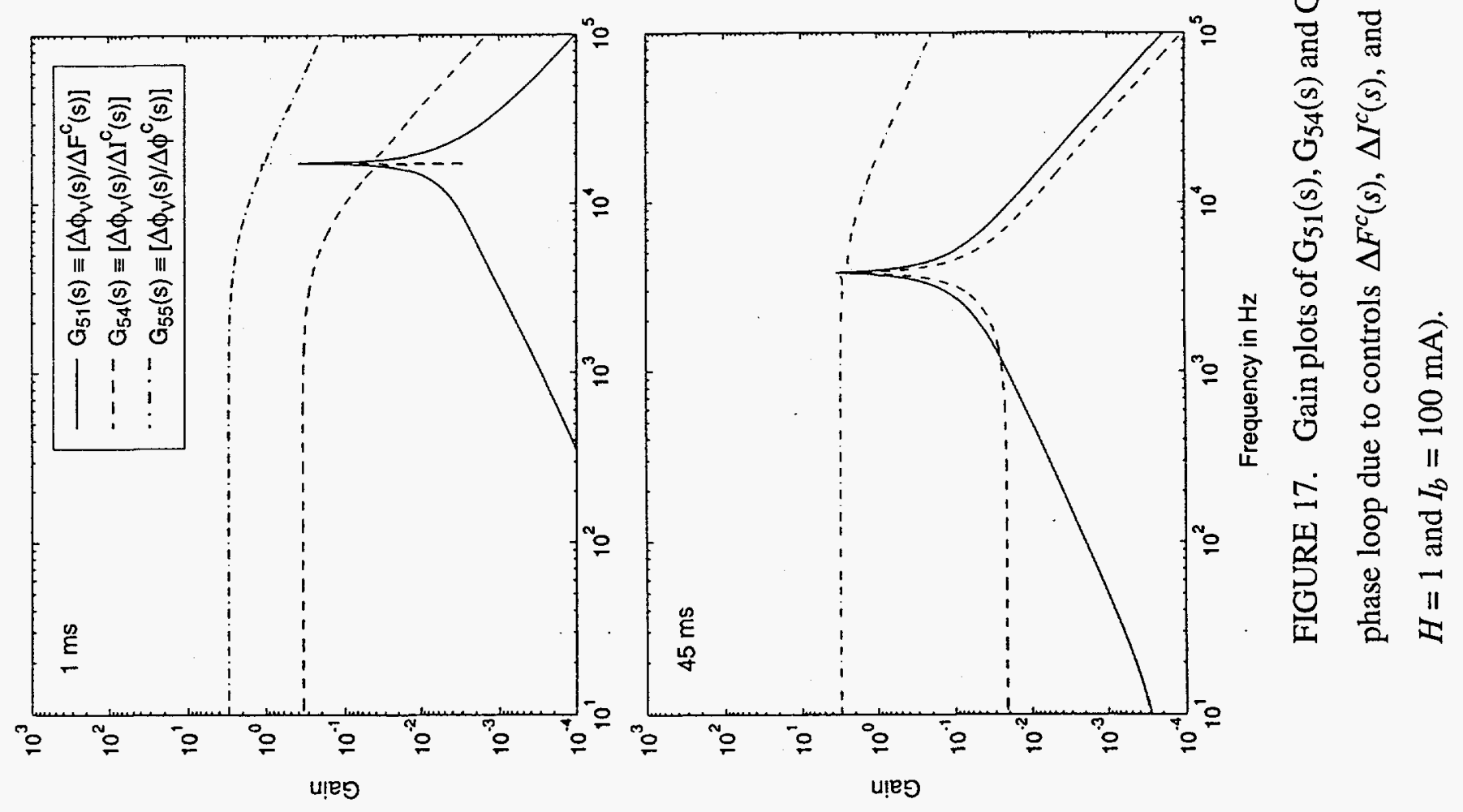



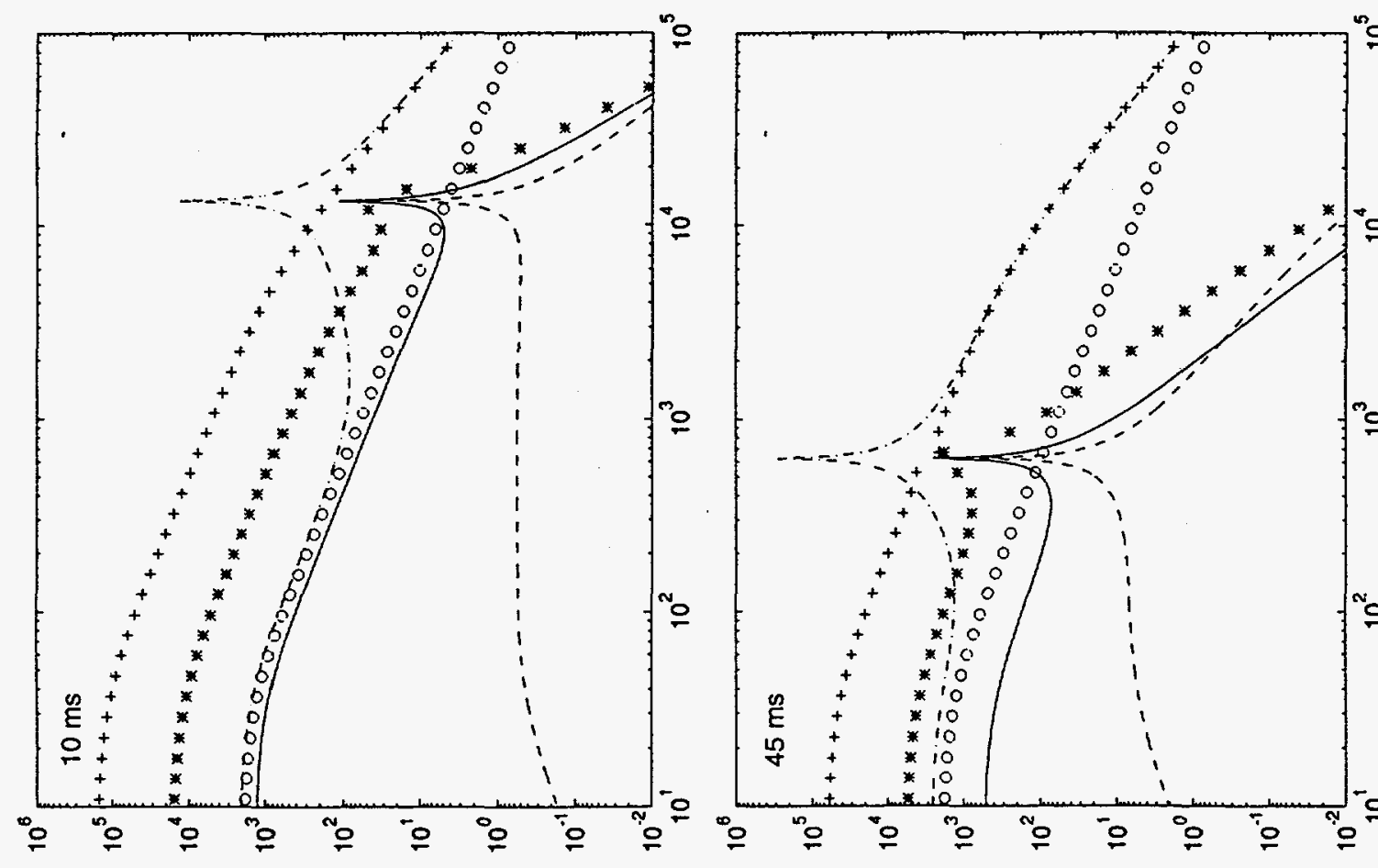

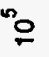
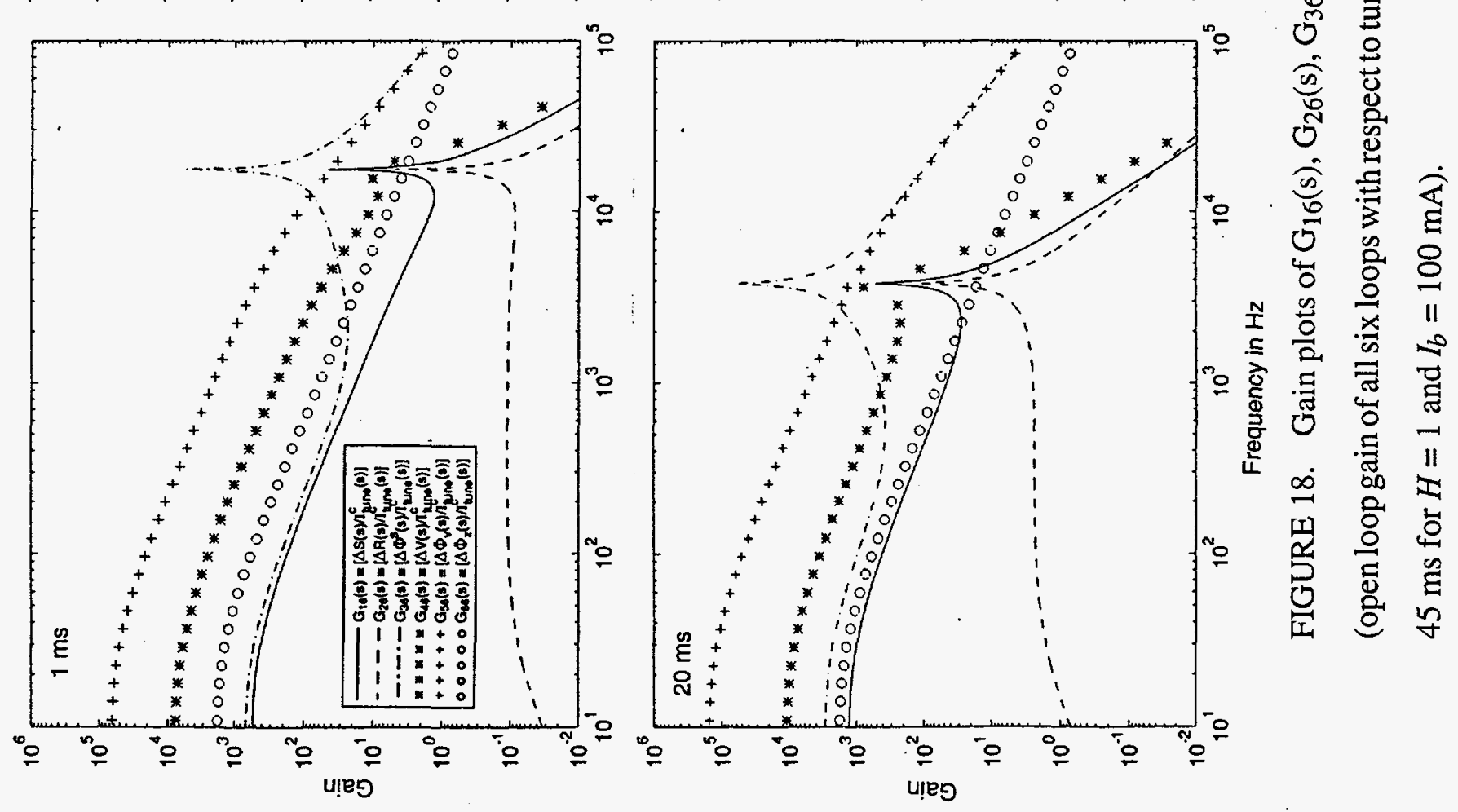

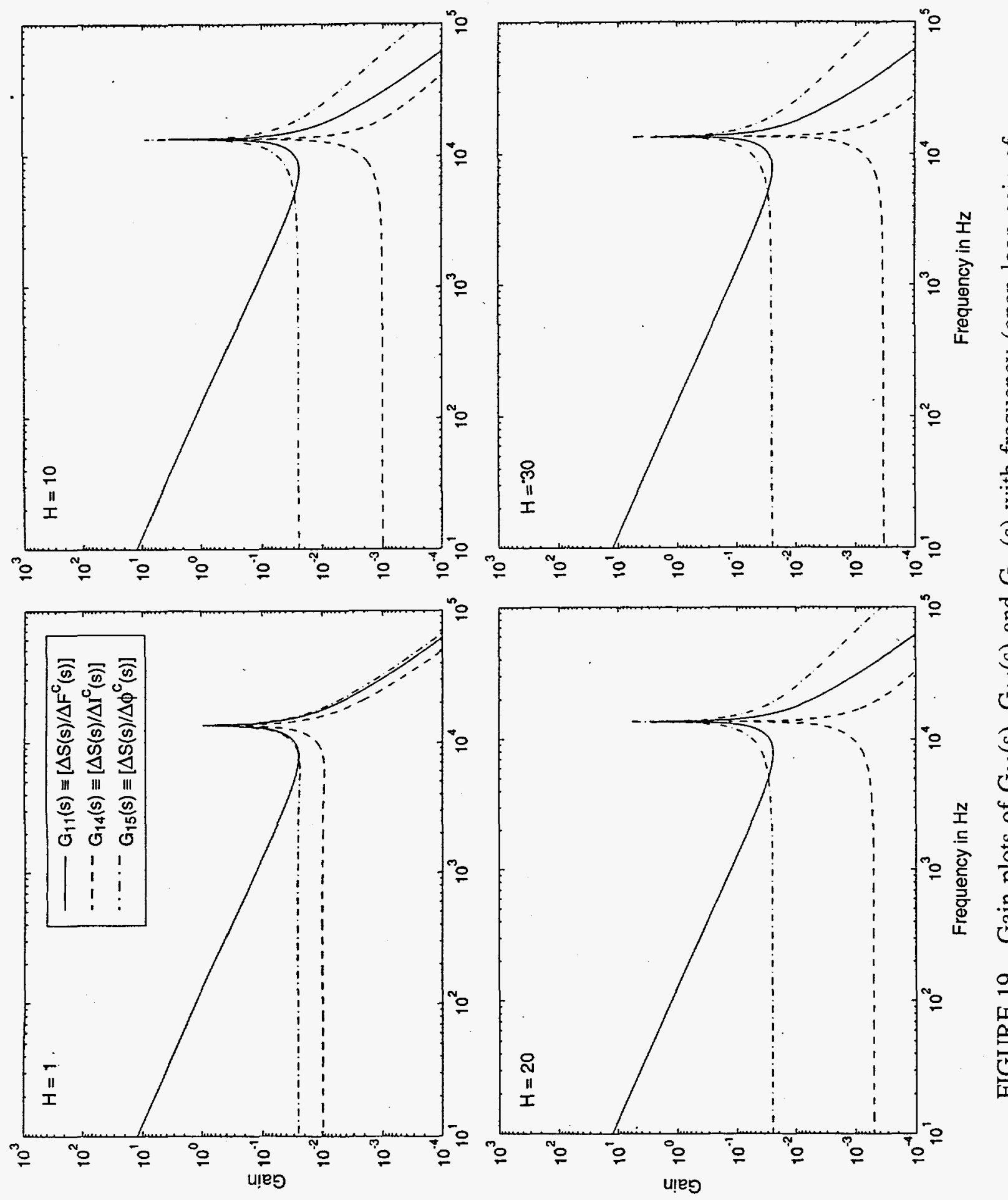

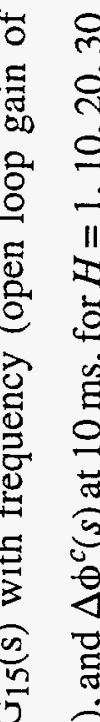

芩

(2)

子

$\stackrel{0}{=} \stackrel{\infty}{0}$

4

48

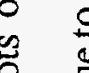

음

吉

․

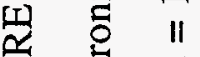

卢 鬲 导 

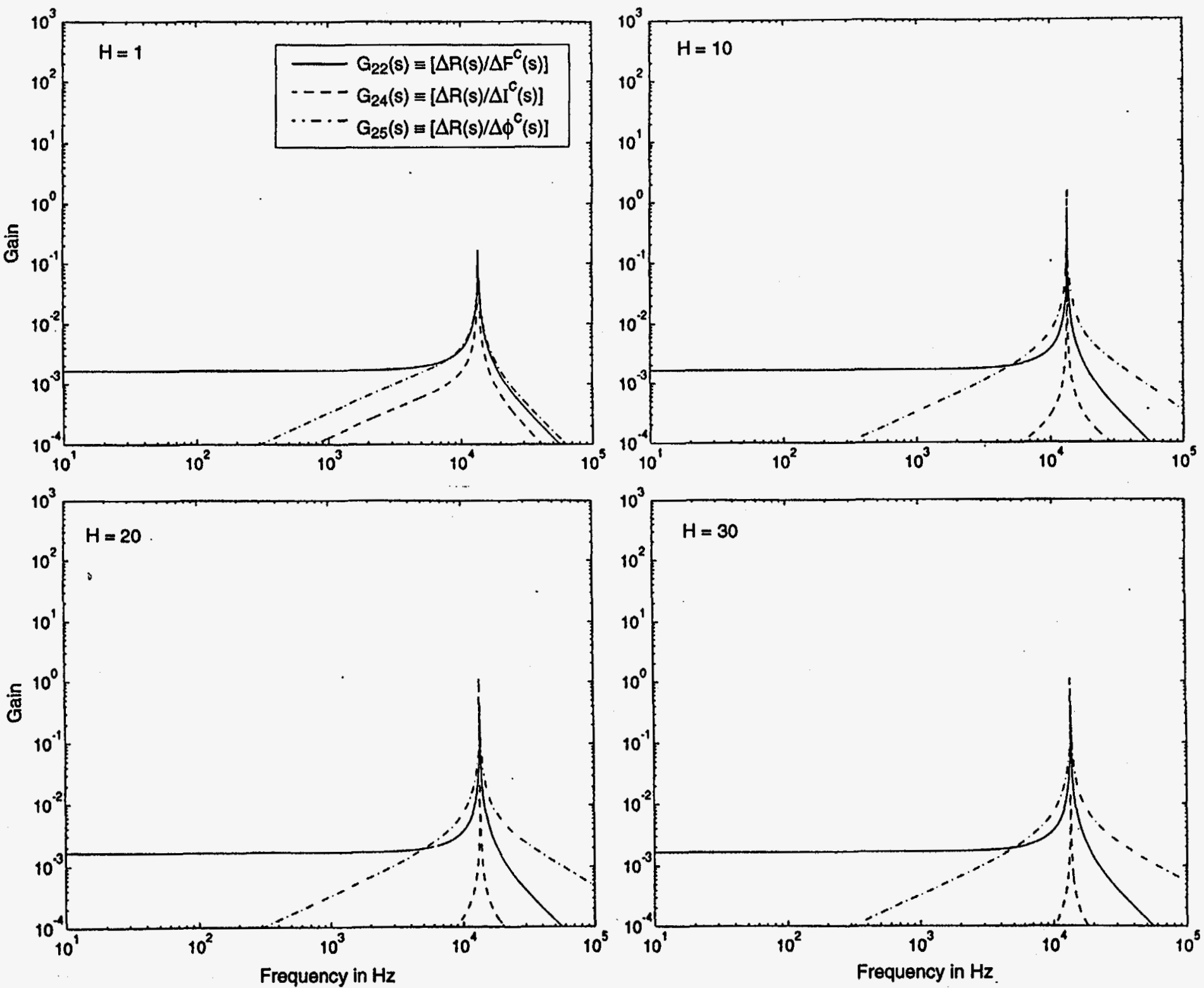

FIGURE 20. Gain plots of $\mathrm{G}_{22}(\mathrm{~s}), \mathrm{G}_{24}(\mathrm{~s})$ and $\mathrm{G}_{25}(\mathrm{~s})$ with frequency (open loop gain of radial loop due to controls $\Delta F^{c}(s), \Delta I^{c}(s)$, and $\Delta \phi^{c}(s)$ at $10 \mathrm{~ms}$, for $H=1,10,20,30$ and $\left.I_{b}=100 \mathrm{~mA}\right)$. 

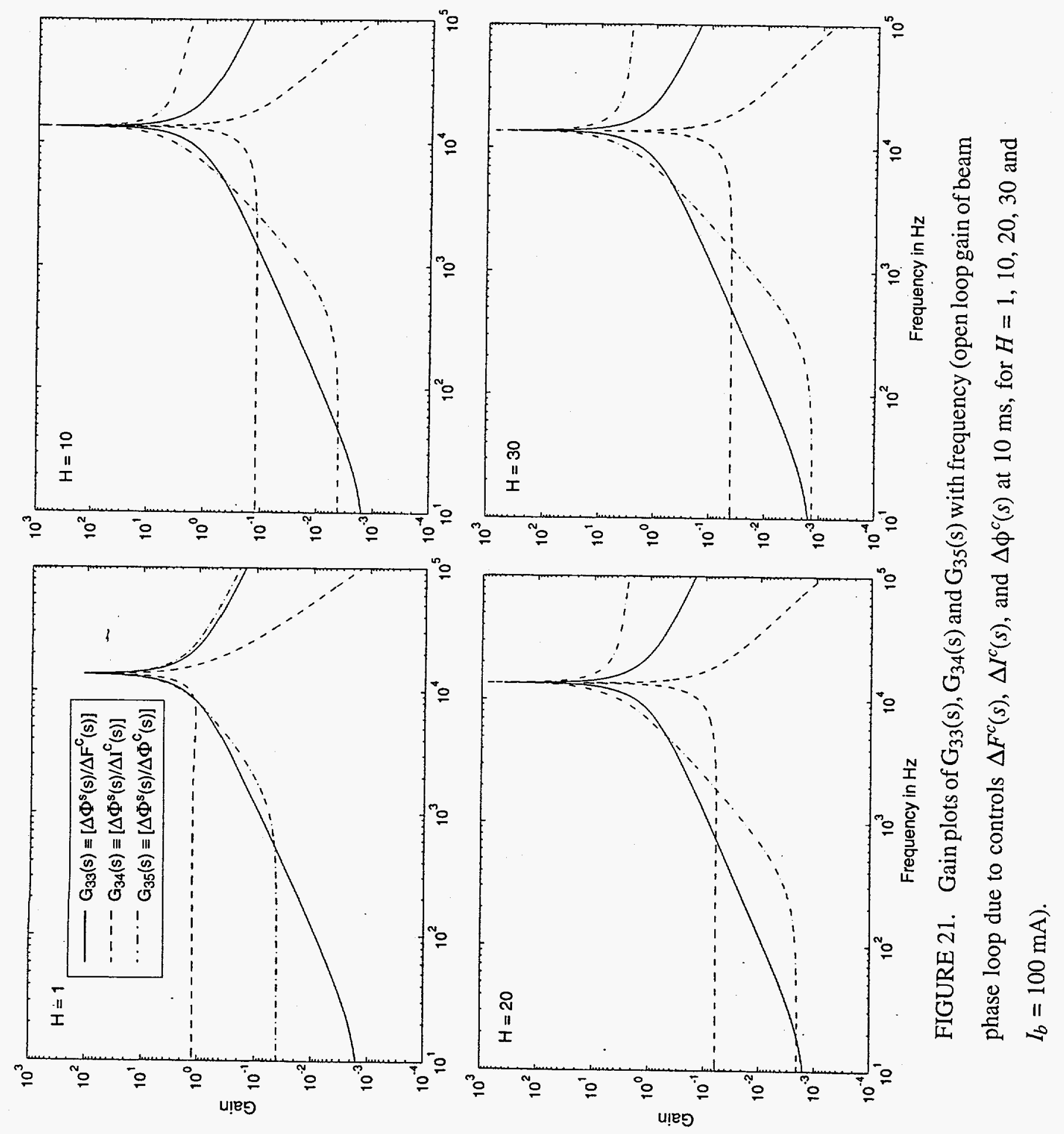


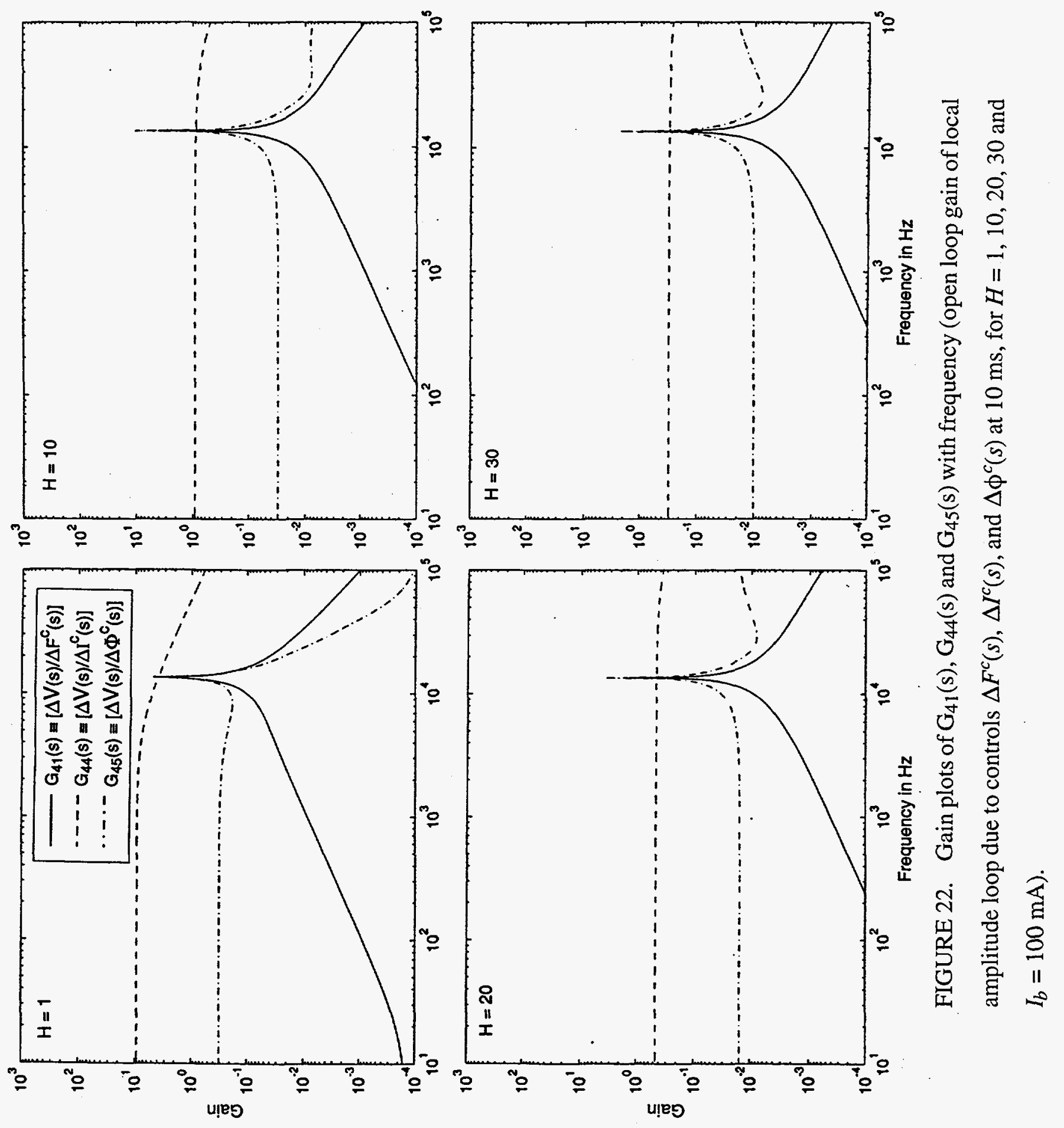



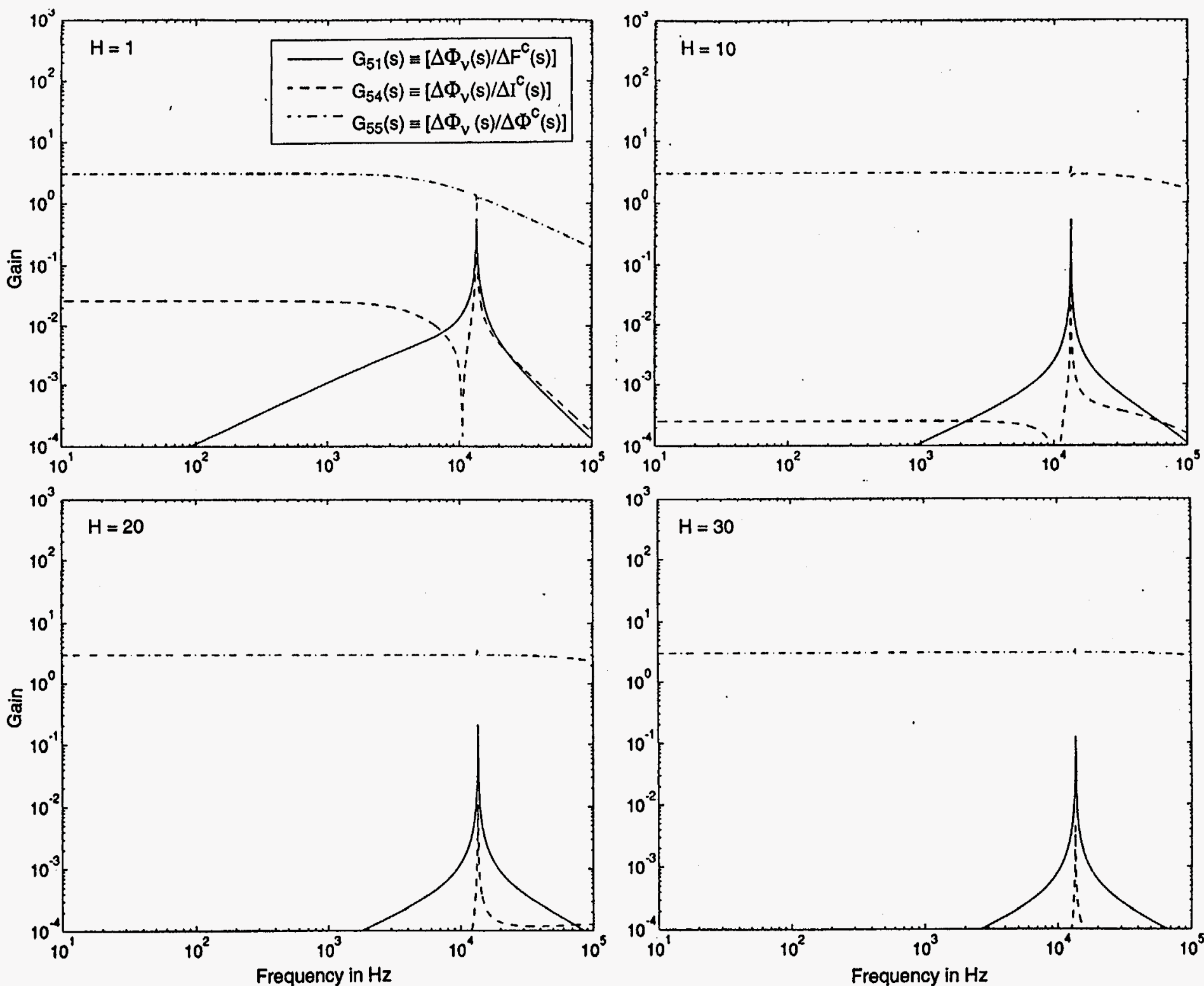

FIGURE 23. Gain plots of $\mathrm{G}_{51}(\mathrm{~s}), \mathrm{G}_{54}(\mathrm{~s})$ and $\mathrm{G}_{55}(\mathrm{~s})$ with frequency (open loop gain of local phase loop due to controls $\Delta F^{c}(s), \Delta I^{c}(s)$, and $\Delta \phi^{c}(s)$ at $10 \mathrm{~ms}$, for $H=1,10,20,30$ and $\left.I_{b}=100 \mathrm{~mA}\right)$. 

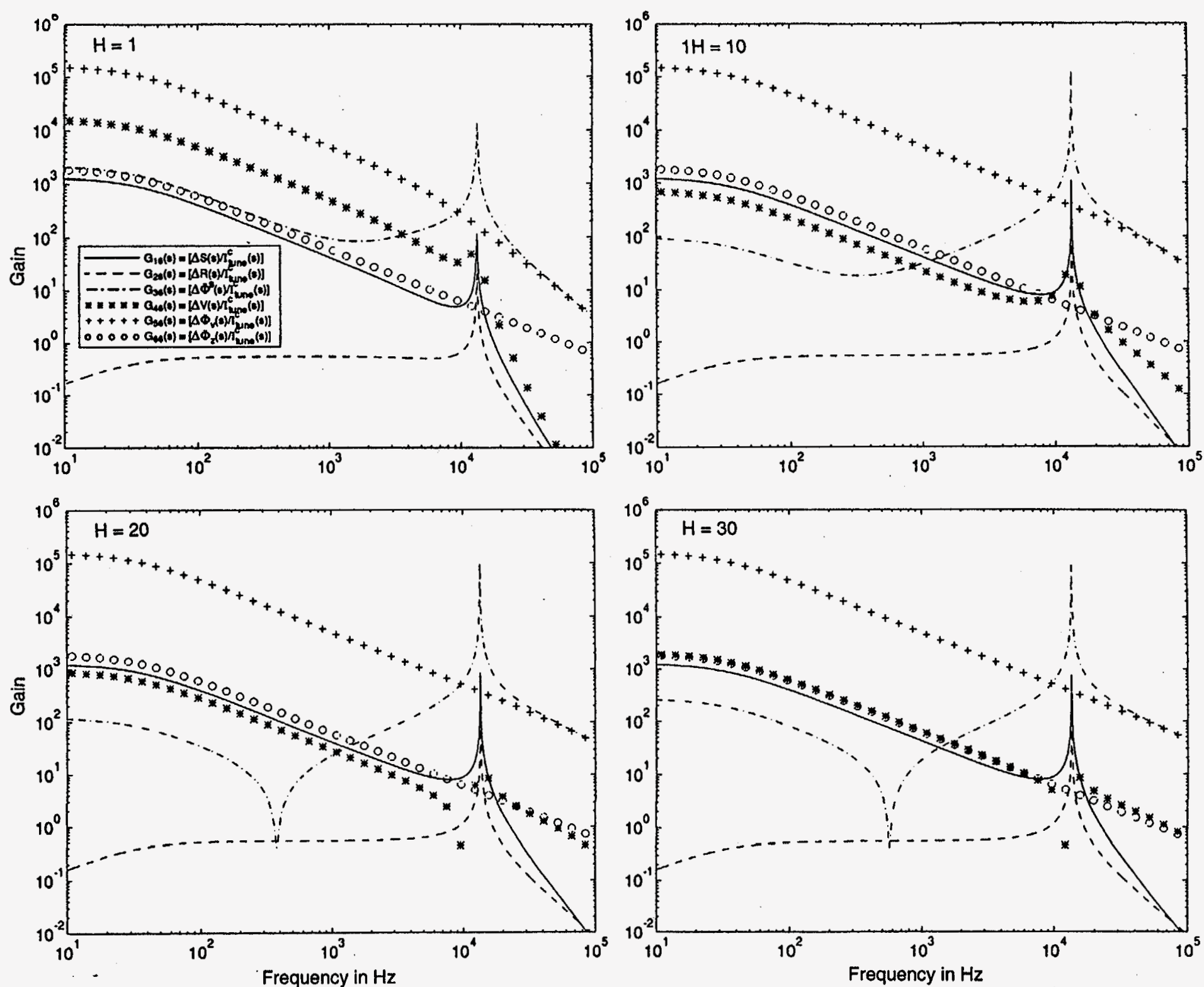

FIGURE 24. Gain plots of $\mathrm{G}_{16}(\mathrm{~s}), \mathrm{G}_{26}(\mathrm{~s}), \mathrm{G}_{36}(\mathrm{~s}), \mathrm{G}_{46}(\mathrm{~s}), \mathrm{G}_{56}(\mathrm{~s})$ and $\mathrm{G}_{66}(\mathrm{~s})$ with frequency (open loop gain of all six loops with respect to tuning control, $I_{\text {tune }}^{c}(s)$ at $10 \mathrm{~ms}$ for $\mathrm{H}=1,10,20$, 30 and $\left.I_{b}=100 \mathrm{~mA}\right)$. 


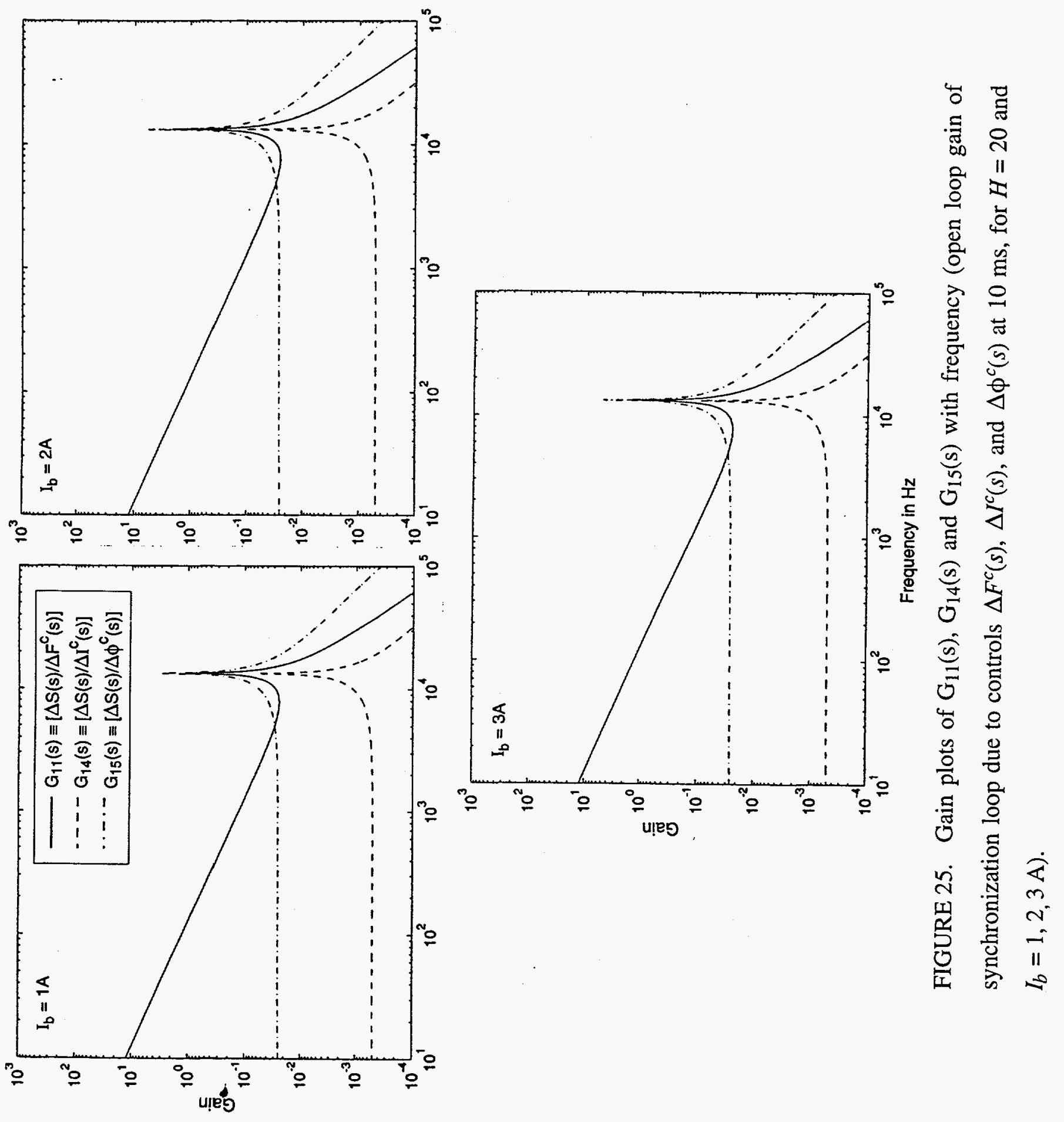



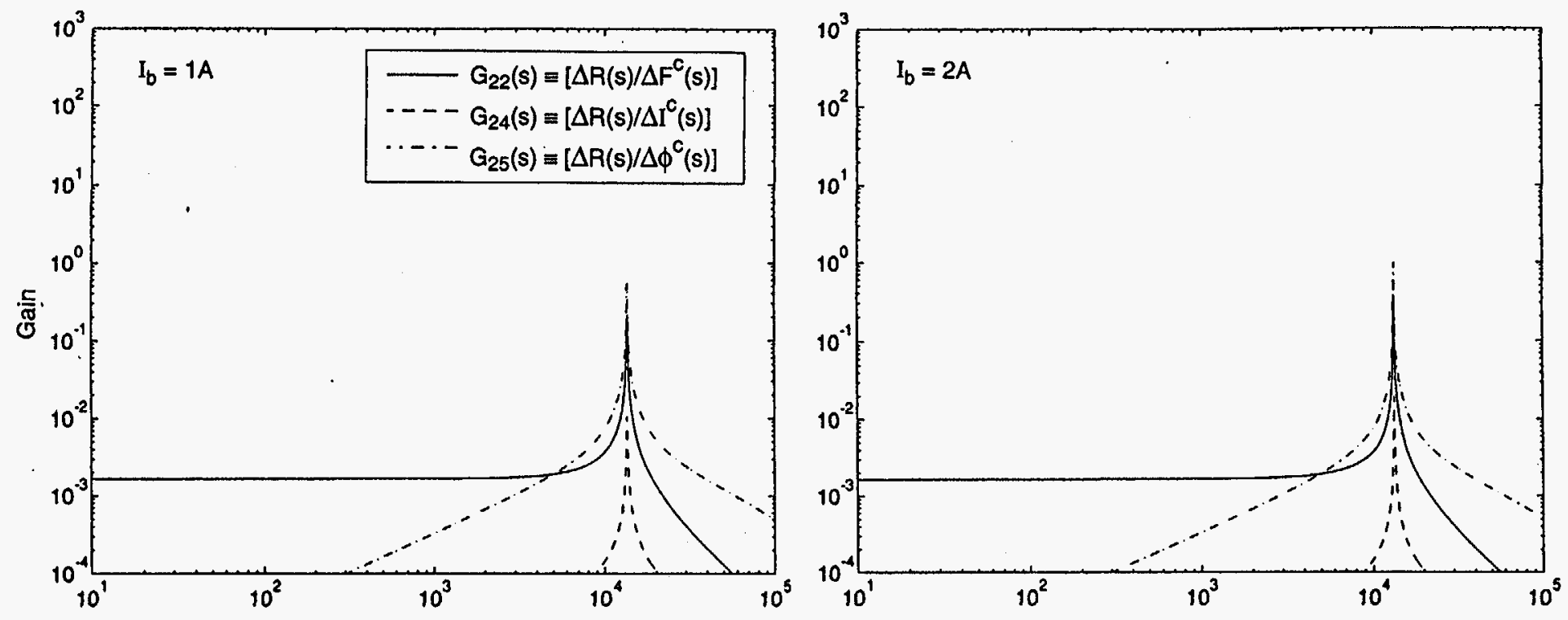

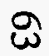

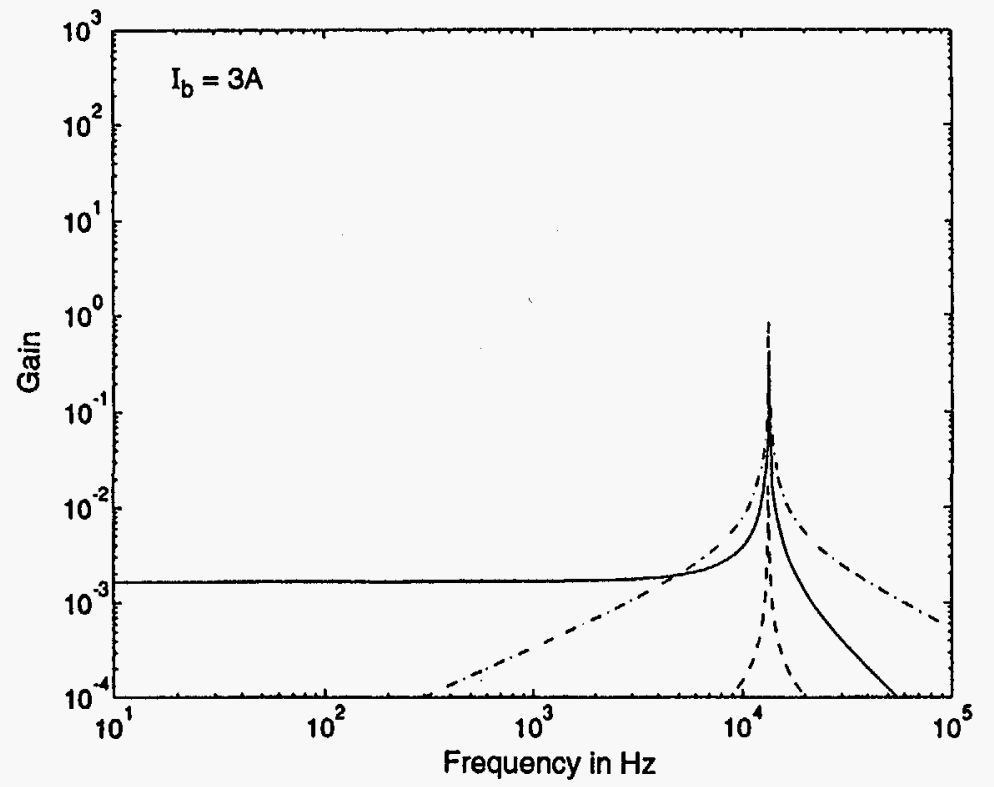

FIGURE 26. Gain plots of $\mathrm{G}_{22}(\mathrm{~s}), \mathrm{G}_{24}(\mathrm{~s})$ and $\mathrm{G}_{25}(\mathrm{~s})$ with frequency (open loop gain of radial loop due to controls $\Delta F^{c}(s), \Delta I^{c}(s)$, and $\Delta \phi^{c}(s)$ at $10 \mathrm{~ms}$, for $H=20$ and $\left.I_{b}=1,2,3 \mathrm{~A}\right)$. 

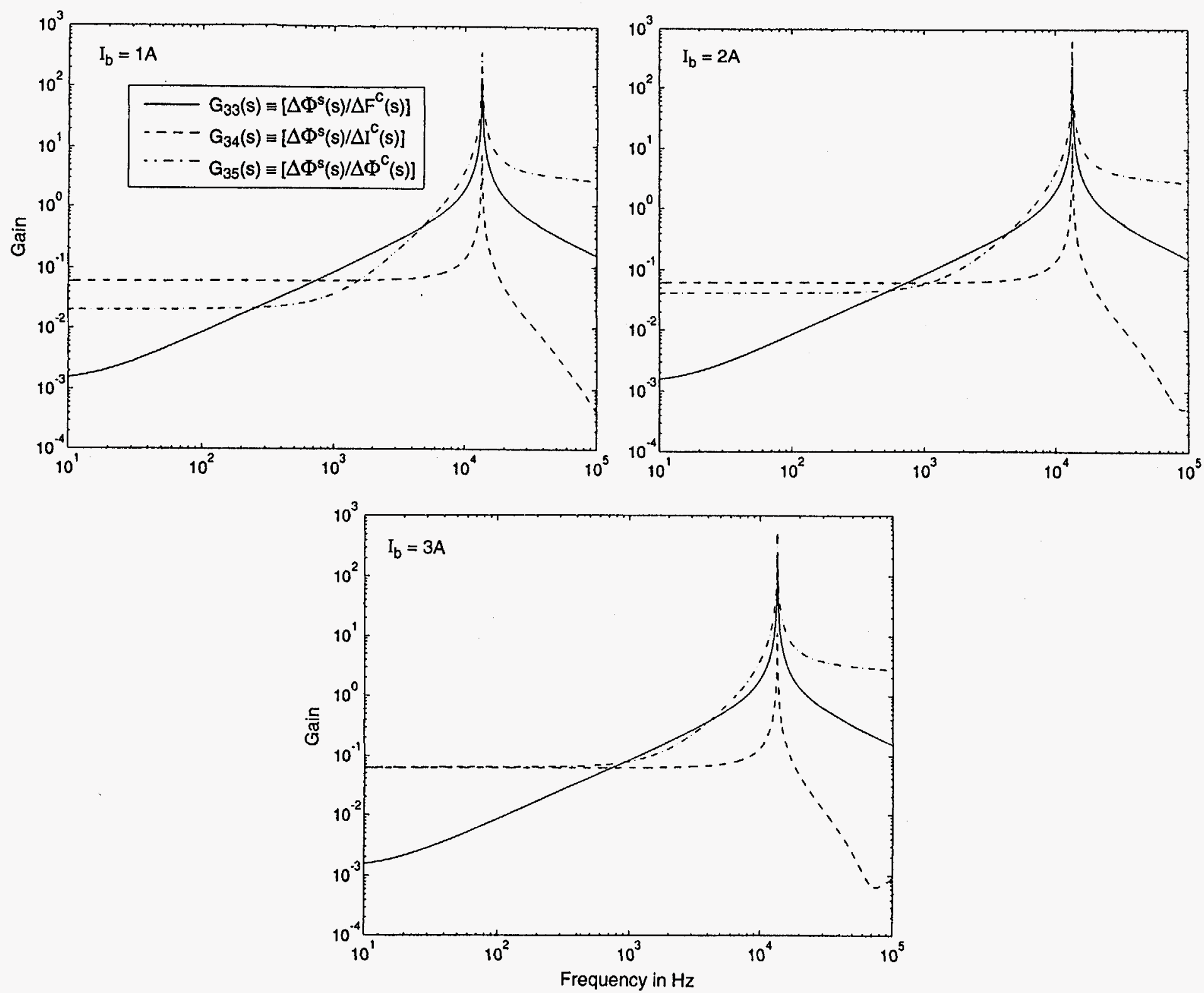

FIGURE 27. Gain plots of $\mathrm{G}_{33}(\mathrm{~s}), \mathrm{G}_{34}(\mathrm{~s})$ and $\mathrm{G}_{35}(\mathrm{~s})$ with frequency (open loop gain of beam phase loop due to controls $\Delta F^{c}(s), \Delta I^{c}(s)$, and $\Delta \phi^{c}(s)$ at $10 \mathrm{~ms}$, for $H=20$ and $\left.I_{b}=1,2,3 \mathrm{~A}\right)$. 

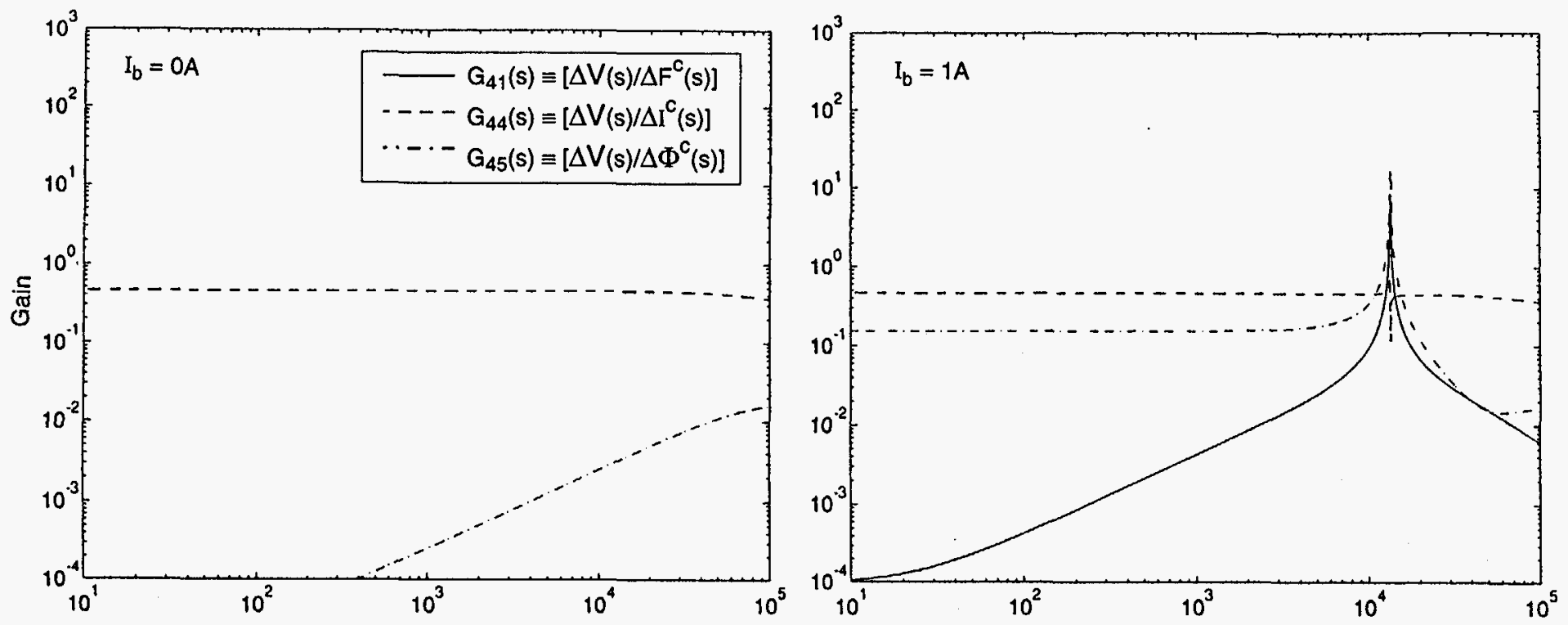

is
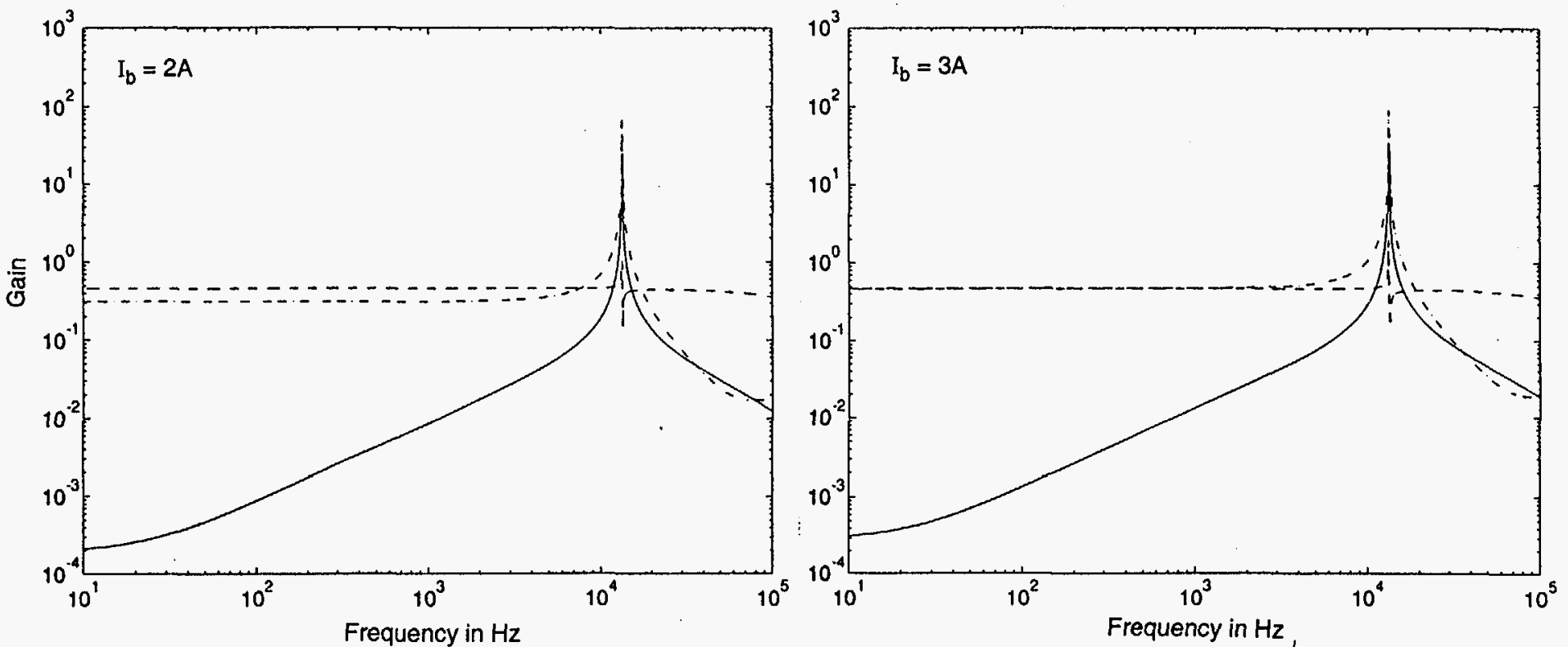

FIGURE 28. Gain plots of $\mathrm{G}_{41}(\mathrm{~s}), \mathrm{G}_{44}(\mathrm{~s})$ and $\mathrm{G}_{45}(\mathrm{~s})$ with frequency (open loop gain of local amplitude loop due to controls $\Delta F^{c}(s), \Delta l^{c}(s)$, and $\Delta \phi^{c}(s)$ at $10 \mathrm{~ms}$, for $H=20$ and $\left.I_{b}=0,1,2,3 \mathrm{~A}\right)$. 

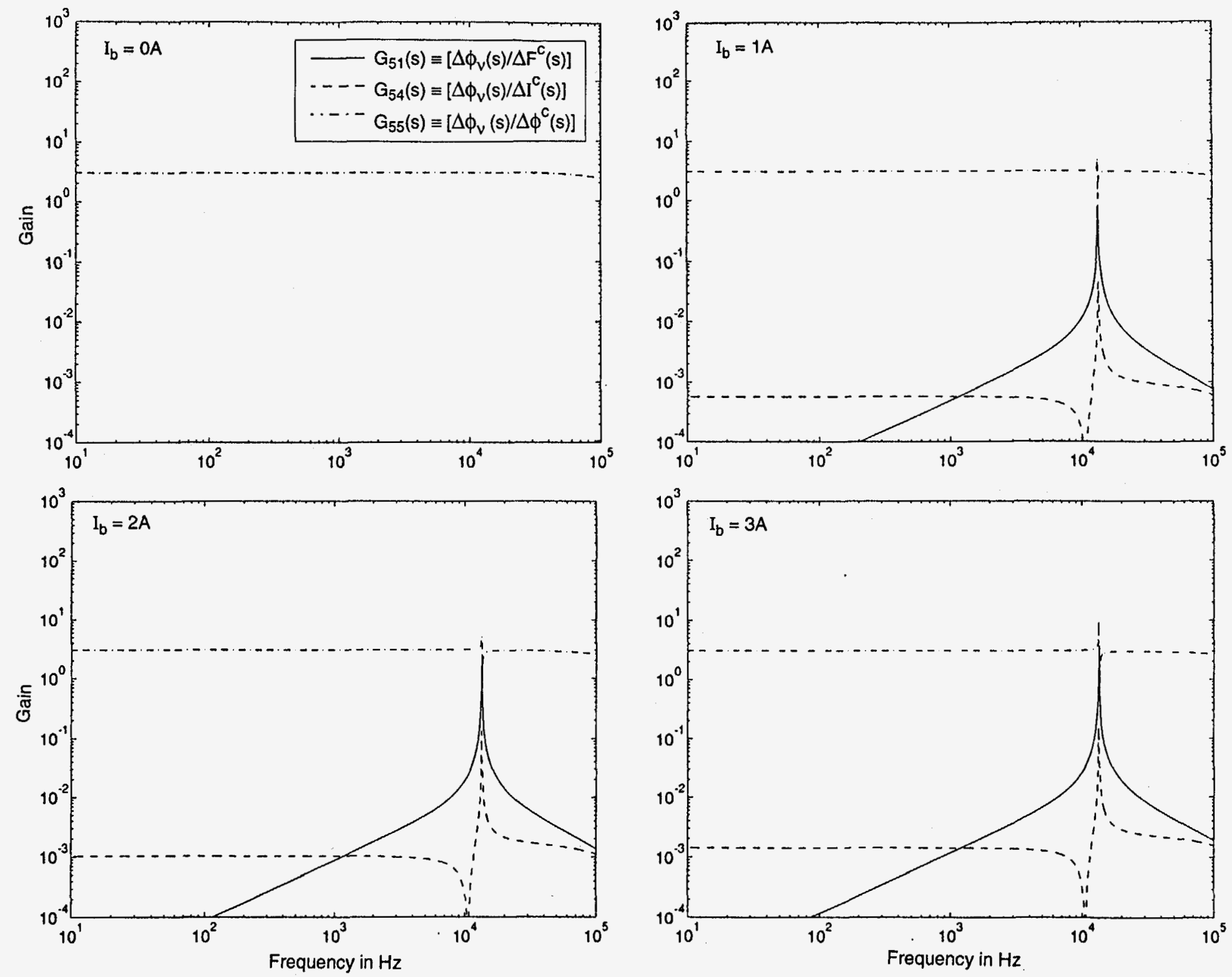

FIGURE 29. Gain plots of $\mathrm{G}_{51}(\mathrm{~s}), \mathrm{G}_{54}(\mathrm{~s})$ and $\mathrm{G}_{55}(\mathrm{~s})$ with frequency (open loop gain of local phase loop due to controls $\Delta F^{c}(s), \Delta I^{c}(s)$, and $\Delta \phi^{c}(s)$ at $10 \mathrm{~ms}$, for $H=20$ and $\left.I_{b}=0,1,2,3 \mathrm{~A}\right)$. 

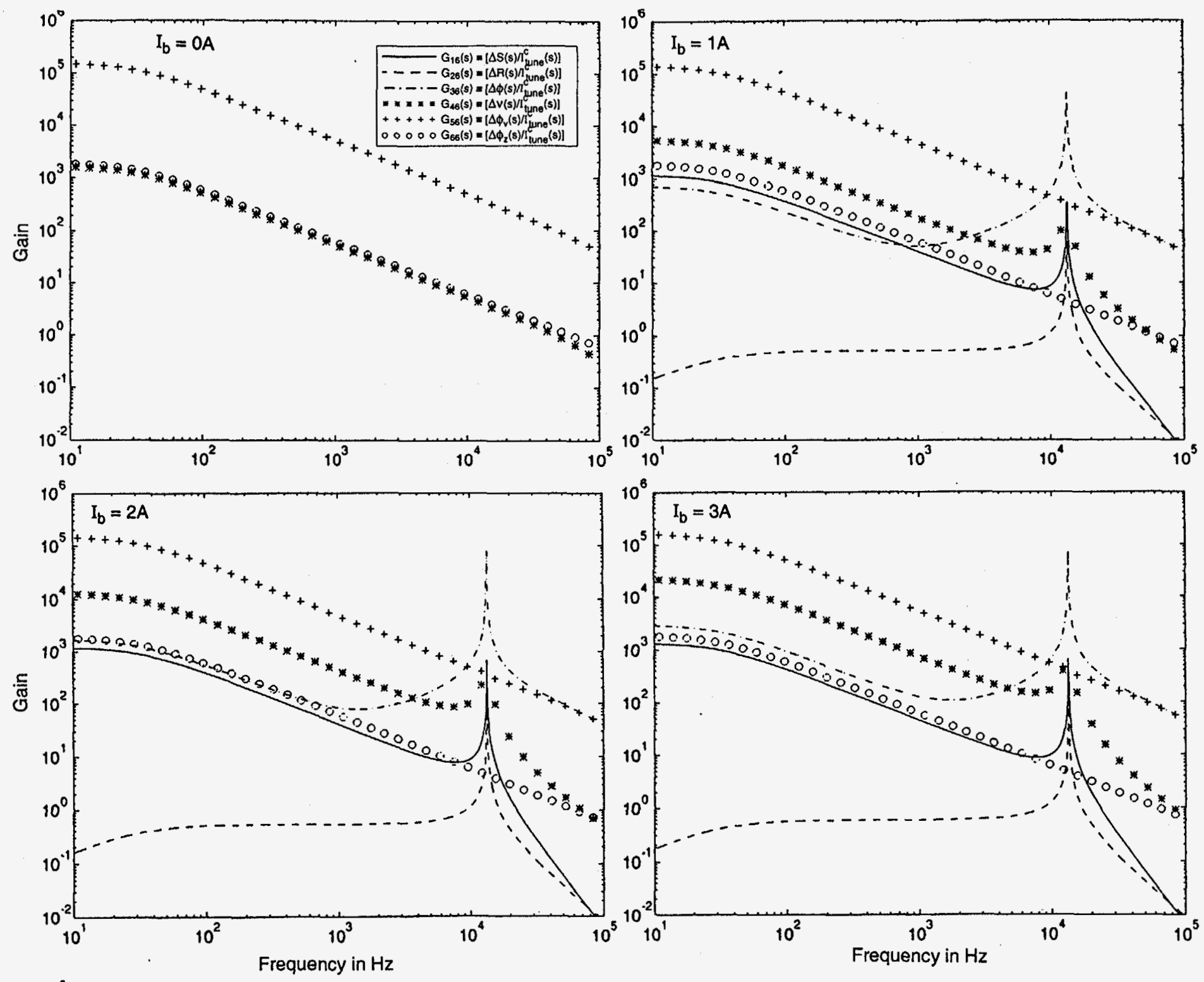

FIGURE 30. Gain plots of $\mathrm{G}_{16}(\mathrm{~s}), \mathrm{G}_{26}(\mathrm{~s}), \mathrm{G}_{36}(\mathrm{~s}), \mathrm{G}_{46}(\mathrm{~s}), \mathrm{G}_{56}(\mathrm{~s})$ and $\mathrm{G}_{66}(\mathrm{~s})$ with frequency (open loop gain of all six loops with respect to tuning control, $I_{\text {tune }}^{c}(s)$ at $10 \mathrm{~ms}$ for $H=20$ and $\left.I_{b}=0,1,2,3 \mathrm{~A}\right)$. 


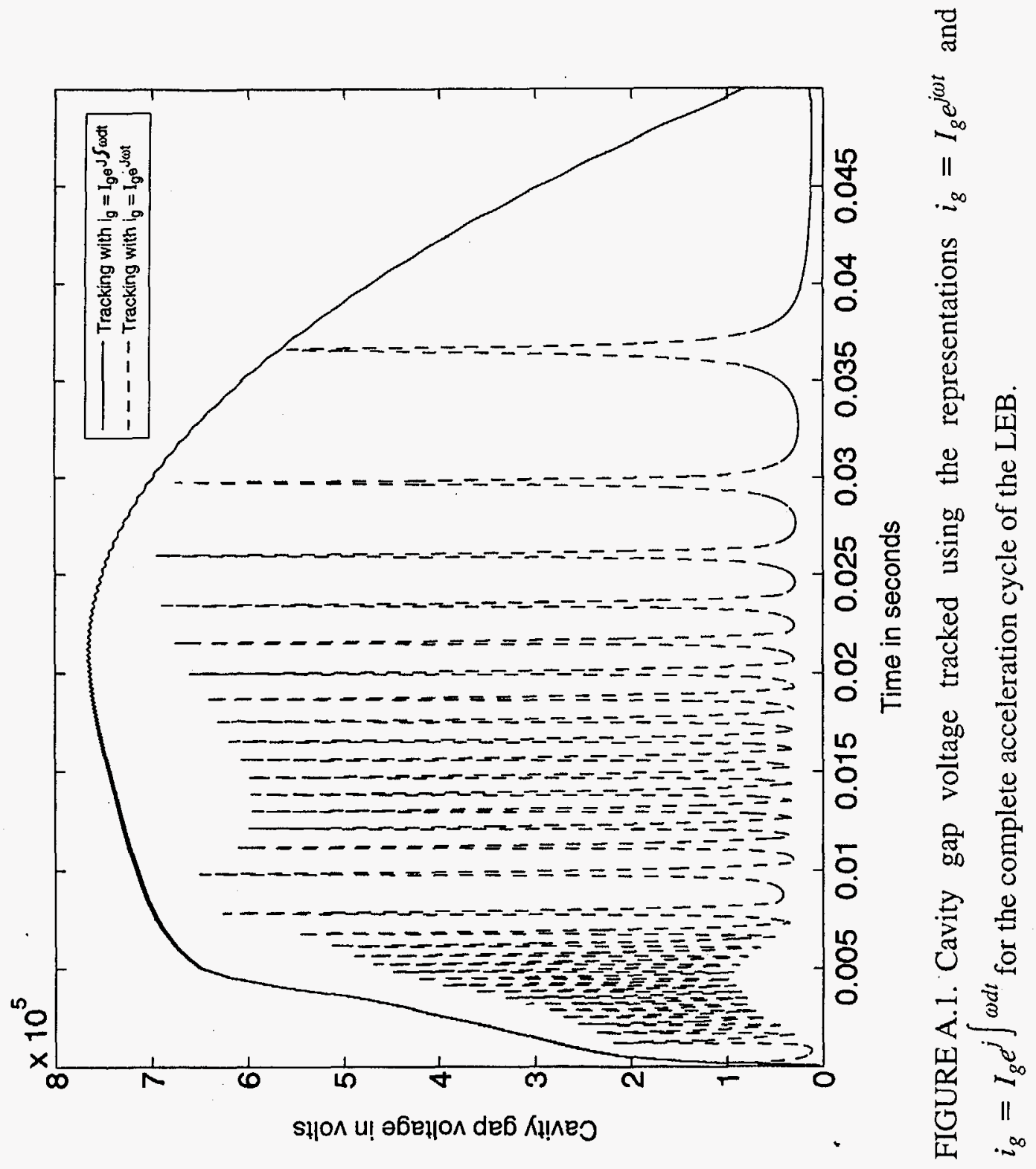

